Universidade de São Paulo

Instituto de Astronomia, Geofísica e Ciências Atmosféricas

Departamento de Astronomia

Rafael Miloni Santucci

\title{
Caracterização de estrelas azuis tardias no campo galáctico
}

São Paulo 



\section{Caracterização de estrelas azuis tardias no campo galáctico}

Dissertação apresentada ao Departamento de Astronomia do Instituto de Astronomia, Geofísica e Ciências Atmosféricas da Universidade de São Paulo como requisito parcial para a obtenção do título de Mestre em Ciências.

Versão corrigida. O volume original encontrase na unidade.

Área de Concentração: Astronomia Orientadora: $\operatorname{Prof}^{\mathrm{a}}$ Dr$^{\mathrm{a}}$ Silvia Rossi

São Paulo 

Aos meus pais, obrigado pelo carinho e atenção, os quais nunca foram tardios nem pobres. 



\section{Agradecimentos}

Aos meus pais, investidores do meu crescimento, intelectual, vertical e horizontal;

A Elisa, pela ajuda, paciência e incentivo nas horas necessárias;

À Prof ${ }^{a}$. Dra. Silvia Rossi, orientadora, co-mãe e companheira de gargalhadas acima de $70 \mathrm{db}$;

Ao "Excelente" Vinicius Placco, co-orientador e co-irmão, um exemplo de organização e competência;

À Dinah, que me cedeu o microcomputador utilizado para realizar esse trabalho;

Aos meus irmãos, Roginho e Rodrigo, por toda a diversão e cobrança, sem as quais esse trabalho jamais seria concluído;

Aos pesquisadores, Timothy Beers e Young Lee por terem me apresentado ao tema das estrelas BS, e pelo suporte com o SSPP;

Ao professor Rama Teixeira, por todo aprendizado que o observatório de Valinhos me proporcionou nesse tempo;

Aos professores Jacques Lepine e Marcos Dias que ministraram as disciplinas de Estrutura da Galáxia e Atmosferas Estelares, Claudia Mendes de Oliveira pela supervisão na monitoria PAE;

A Ana e Luciene pela ajuda nos estudos, sempre gentilmente oferecida;

Às colegas de sala Viviane, Nadili e Aiara pela boa convivência;

Aos colegas, Márcio, Marcus (Caçapava), Gleidson, Felipe(s), Oscar, Bruno Dias, Phillip, Douglas, Alan, Rogério, Thaíse, Andressa(s), Gil(derlon), Lívia, Dai, Elisa (Li), Tio Chico, Vinaum, Marcelo Rubinho, Budda, Minininho, Vitor, Teresa, Moisés e Zé Colméia, listados em ordem browniana;

Aos funcionários do IAG e equipes de apoio, por fornecer um ótimo ambiente de tra- 
balho;

À CAPES, pelo apoio financeiro.

À todos que eu não citei por problemas mentais momentâneos ou àqueles que deixei de dar a devida atenção enquanto estive realizando esse trabalho, minhas sinceras desculpas e meu muito obrigado pela compreensão!

Esta dissertação foi escrita em $\mathrm{LT}_{\mathrm{E}} \mathrm{X}$ com a classe IAGTESE, para teses e dissertações do IAG. 
"Se enxerguei mais longe, foi porque uso óculos, pois tenho miopia."

Rafael Miloni Santucci

"Um cientista deve, acima de tudo, ser como uma criança (...) não dá pra ser um cientista se você vai ficar se preocupando se as pessoas vão ou não te achar bobo."

Douglas Adams 



\section{Resumo}

As estrelas azuis tardias (blue straggler stars - estrelas BS) são estrelas de sequência principal que apresentam um aparente atraso evolutivo em relação às suas vizinhanças. Elas foram identificadas inicialmente na sequência principal de aglomerados globulares acima do ponto de turnoff no diagrama HR. Desde então, têm sido encontradas em todos os ambientes estelares: aglomerados abertos e globulares, galáxias anãs próximas e entre as estrelas de campo na Galáxia.

O principal objetivo deste trabalho é construir uma grande amostra de estrelas BS no campo galáctico. Para tanto, métodos de separação desses objetos de estrelas BHB foram comparados com critérios de seleção que envolvem parâmetros atmosféricos estimados pelo SEGUE Stellar Parameter Pipeline (SSPP). Tal procedimento permitiu incluir também estrelas com magnitudes $g>18$, antes excluídas pelos métodos tradicionais para objetos com razão $\langle\mathrm{S} / \mathrm{N}\rangle$ menores que 9 .

Os métodos apresentados neste trabalho permitiram a seleção de uma amostra de 8001 candidatas a estrelas BS que foram analisadas cinematicamente, através de suas velocidades radiais. Verificou-se que $\sim 20 \%$ dessa amostra ( 1000 objetos) possui características que a associa à corrente de Sagitário, sugerindo uma origem extragaláctica para tais objetos. 



\section{Abstract}

Blue straggler stars (BSS) are main sequence stars that exhibit an apparent evolutionary delay over the region where they are found in the HR diagram. They were initially identified in the main sequence of globular clusters above the turnoff point. Since then, they have been found in many different stellar environments: globular and open clusters, near dwarf galaxies, and also in the field of the Galaxy.

The main goal of this work is to build a large sample of BSS in the galactic field region. In order to accomplish this task, classical methods of separation of BSS from BHB stars were compared with proposed restrictions based on their atmospheric parameters, which are estimated by the SEGUE Stellar Parameter Pipeline (SSPP). The latter procedure allowed to include stars with magnitudes $g>18$, not handled by traditional methods when $\langle\mathrm{S} / \mathrm{N}\rangle$ ratio is smaller than 9.

The selection methods gathered a sample of 8001 BS stars. These stars were kinematically analyzed through their radial velocities. The results suggest that many of them $(\sim 1000)$ can have extragalactic origin, associated with Sagittarius stream. 



\section{Lista de Figuras}

1.1 Diagrama HR do aglomerado globular M55. As estrelas azuis tardias (círculos abertos) são identificadas na sequência principal dentro do trapézio. O losango mostra estrelas na sequência principal, antes do ponto de turnoff. Vê-se claramente que as estrelas BS localizam-se no prolongamento da sequência principal. Figura retirada de Sollima et al. (2008). . . . . . . . . . . . . 28

1.2 Comparação entre a densidade superficial de estrelas BS observada (linha azul) e a densidade superficial de estrelas BS simulada (linha vermelha) para o algomerado globular 47 Tuc. Na parte superior vê-se a simulação somente com contribuição do processo de formação de estrelas BS por colisões e, na parte inferior, a simulação com processo colisional e com tranferência de massa de sistemas binários. Ambas as distribuições são normalizadas: no eixo x com respeito ao raio do núcleo do aglomerado $\left(\mathrm{r}_{\text {core }}\right)$ e no eixo y em relação ao número de estrelas de ramo horizontal (horizontal branch - HB) catalogadas por Ferraro et al. (2004). Figura retirada de Mapelli et al. (2004). 31

1.3 Razão $[\mathrm{O} / \mathrm{Fe}]$ em função de $[\mathrm{C} / \mathrm{Fe}]$ para as estrelas azuis tardias observadas no aglomerado globular 47 Tuc. Estrelas BS "normais" são marcadas com círculos vazios, enquanto que 6 estrelas BS com menos Carbono e Oxigênio são marcadas com quadrados. Seus nomes estão indicados. A região cinza corresponde ao local de outras estrelas do ponto de turnoff do aglomerado 47 Tuc. Figura retirada de Ferraro et al. (2006). . . . . . . . . . . . . . . 32 
1.4 Relação entre o índice de cor desavermelhado $(B-V)_{0}$ vs metalidade para estrelas de sequência principal pobres em metais do campo (cruzes pretas) e, para 5 estrelas BS encontradas por Carney et al. (2005) (círculos vermelhos). Também são mostradas 6 estrelas BS binárias do trabalho de Carney et al. (2001) (círculos azuis), além de 7 estrelas BS que apresentaram solução orbital binária com período maior que 20 dias no trabalho de Preston e Sneden (2000) (quadrados preenchidos em azul).A estrela BD+25 1981, que pode ser binária, é graficada como um círculo azul aberto. A linha sólida representa um ajuste às regiões de cor onde se encontram os pontos de turnoff da sequência principal de aglomerados globulares, e a linha tracejada identifica o seu equivalente teórico. Figura retirada de Carney et al. (2005).

2.1 Coordenadas equatoriais das estrelas analisadas neste trabalho na região observada pelo telescópio do SDSS . . . . . . . . . . . . . .

2.2 Dados do DR8 e sua distribuição no diagrama cor-cor, a região destacada em vermelho destaca onde são encontradas as estrelas do tipo A. Os limites dessa região são: $0.90<(u-g)<1.40$ e $-0.25<(g-r)<0.00$. Figura retirada de Deason et al. (2011) . . . . . . . . . . . . . . .

2.3 Região do diagrama cor-cor adotada para espectros do DR8: $0.80<(u-$ $g)<1.40 \mathrm{e}-0.300<(g-r)<0.015 \ldots \ldots \ldots \ldots$

2.4 Quatro exemplos de espectros selecionados via inspeção visual. Com diferentes $\langle\mathrm{S} / \mathrm{R}\rangle \ldots \ldots \ldots \ldots \ldots \ldots \ldots \ldots \ldots \ldots \ldots \ldots \ldots \ldots \ldots \ldots \ldots \ldots$

2.5 Média dos erros associados a fotometria na banda $g$. Os espectros selecionados encontram-se no intervalo $12<g<20 \ldots \ldots \ldots$

2.6 Distribuição das estrelas selecionadas do DR8 em coordenadas celestes galácticas.

Os pontos estão coloridos de acordo com o sinal da velocidade radial heliocêntrica $\left(V_{R_{\odot}}\right.$ em $\left.\mathrm{km} / \mathrm{s}\right): V_{R_{\odot}}<0$ em azul e $V_{R_{\odot}}>0$ em vermelho. . . .

2.7 Distribuição de metalicidade da amostra com parâmetros calculados pelo SSPP. O corte para $[\mathrm{Fe} / \mathrm{H}]=-0.4$ é identificado pela linha azul tracejada. 
2.8 Espectro normalizado de uma estrela gigante (em azul) e de uma estrela anã (em verde) para uma mesma temperatura efetiva ( $~ 8100 \mathrm{~K})$ na região $\mathrm{H}_{\gamma}-\mathrm{H}_{\delta}$. A linha tracejada no nível $20 \%$ abaixo do contínuo mostra a diferença na largura das asas, quanto maior a gravidade superficial, maior será a largura da asa. . . . . . . . . . . . . . . . . . . . . .

3.1 Distribuição da razão $\langle\mathrm{S} / \mathrm{R}\rangle$ dos espectros utilizados. A linha tracejada azul mostra o limite em $\langle\mathrm{S} / \mathrm{R}\rangle$ acima do qual o método $\mathrm{D}_{0.2}$ é aplicável. . . . . 46

3.2 Espectros da Figura 2.4 normalizados e com intensidade de fluxo deslocada. Com o nível do contínuo constante, os ajustes se Sérsic tem erros menores.

3.3 Distribuição normal dos parâmetros $a$, calculados pelo perfil de Sérsic, para as diferentes linhas do H. . . . . . . . . . . . . . . . .

3.4 Ajuste de Sérsic na região da série de Balmer. A linha preta representa o espectro observado, a linha vermelha mostra o ajuste do perfil de Sérsic (para $\langle a\rangle$ fixo) e a linha azul tracejada mostra o nível 20\% abaixo do contínuo. 49

3.5 Gráfico dos parâmetros $\mathrm{f}_{m}$ vs $\mathrm{D}_{0.2}$ para as linhas $\mathrm{H}_{\alpha}, \mathrm{H}_{\beta}, \mathrm{H}_{\gamma}$ e $\mathrm{H}_{\delta}$ da série de Balmer. Dentre as linhas avaliadas, aquela que apresenta a maior distinção entre dois grupos de estrelas é a $\mathrm{H}_{\delta} \ldots \ldots \ldots$. . . . . . . . . . . 50

3.6 Gráfico dos parâmetros $\mathrm{f}_{m \delta}$ vs $\mathrm{D}_{0.2 \delta}$ com magnitude $g<18$. Nota-se que a dispersão diminui em virtude da limitação de objetos menos brilhantes, cuja razão $\langle\mathrm{S} / \mathrm{R}\rangle$ é menor. O retângulo em vermelho é povoado por estrelas de tipo F, G e K. A região comum às estrelas gigantes BHB é vista na área azul e a região superior, em verde, contém as candidatas a estrelas BS. . .

3.7 Parâmetros de forma e largura ( $c$ vs b) para as linhas $\mathrm{H}_{\alpha}, \mathrm{H}_{\beta}, \mathrm{H}_{\gamma}$ e $\mathrm{H}_{\delta}$. A linha $\mathrm{H}_{\alpha}$ é incapaz de mostra qualquer distinção entre estrelas anãs e gigantes. 52

3.8 Média dos parâmetros forma e largura ( $c$ vs $b$ ) para as linhas $\mathrm{H}_{\beta}, \mathrm{H}_{\gamma}$ e $\mathrm{H}_{\delta}$. Os pontos são coloridos de acordo com as regiões ressaltadas na Figura 3.6. Pontos verdes representam as estrelas classificadas como azuis tardias (BS), pontos azuis as estrelas gigantes (BHB) e os pontos vermelhos as estrelas de tipo F, G e K. 
3.9 Relação entre os parâmetros atmosféricos estimados pelo SSPP do SDSS. Pode-se notar dois grupos de estrelas concentrados na região $\mathrm{T} \geq 7500 \mathrm{~K}$ : um com $\log (\mathrm{g})$ típico de gigantes $\sim 3.4$ dex e outro com $\log (\mathrm{g})$ típico de estrelas na sequência principal $\sim 4.4$ dex. . . . . . . . . . . . . .

3.10 Distribuição de $\log (\mathrm{g})(g<18)$ de parâmetros fornecidos pelo SSPP. O pico centrado em $\log (\mathrm{g})=3.385(\sigma=0.138)$ representa a distribuição de estrelas gigantes e $\log (\mathrm{g})=4.333(\sigma=0.188)$ as estrelas anãs. . . . . . . . . .

3.11 Relação entre os parâmetros $\mathrm{T}_{\text {ef }}$ e $\log (\mathrm{g})$ estimados pelo SSPP. A região do gráfico para $\log (\mathrm{g})>3.8$ e $\mathrm{T}_{e f}>7500 \mathrm{~K}$ limita a área comum de estrelas do tipo A anãs (BS). Os pontos verdes representam as estrelas classificadas como BS pelo método do SSPP, em azul estão representadas as estrelas gigantes (BHB) e em vermelho as estrelas mais frias que $7500 \mathrm{~K}$. . . . . .

3.12 Gráfico dos parâmetros $\mathrm{f}_{m \delta}$ vs $\mathrm{D}_{0.2 \delta}$ colorido de acordo com as restrições adotadas pelo SSPP. Vemos que as dispersões encontradas para as estrelas gigantes e anãs se sobrepõe além dos limites adotados pelo método $\mathrm{f}_{m \delta}$ vs

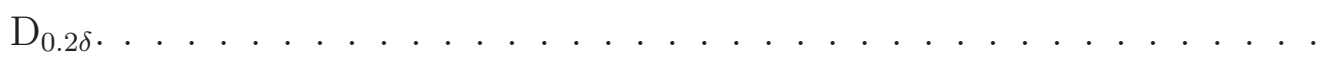

3.13 Relação entre os parâmetros $c_{\beta \gamma \delta}$ vs $b_{\beta \gamma \delta}$ coloridos de acordo com restrições adotadas pelo SSPP. O método que utiliza as restrições do SSPP se mostra mais eficiente para separar as estrelas anãs de gigantes no método de forma e largura, pois suas restrições mostram contaminações menores entre os ramos (anãs e gigantes) do que quando utilizamos as restrições do método $\mathrm{f}_{m \delta}$ vs

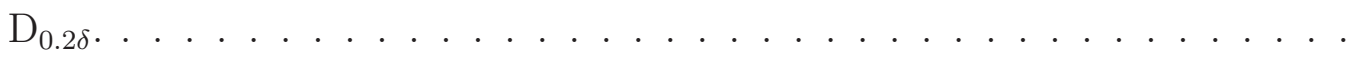

3.14 Região do diagrama cor-cor colorida de acordo com a classificação do SSPP: Os pontos em verde representam as estrelas anãs candidatas a estrelas BS, em azul estão representadas as estrelas BHB e em vermelho estrelas de tipo espectral F, Ge K. . . . . . . . . . . . . . . . . 57

3.15 Relação entre os parâmetros $\mathrm{T}_{e f}$ vs $\mathrm{f}_{m \delta}$. Nota-se que há uma nítida correlação entre a temperatura efetiva estimada pelo SSPP e o parâmetro relacionado com a medida do fluxo no centro da linha em relação ao contínuo. 
3.16 Relação entre os parâmetros $\mathrm{D}_{0.2 \delta}$ e $b_{\beta \gamma \delta}$. Há uma nítida correlação entre ambos. Entretanto, a comparação entre eles não revela diferença entre estrelas anãs e gigantes. . . . . . . . . . . . . . . . . .

3.17 Relação entre os parâmetros $\log (\mathrm{g})$ vs $b_{\beta \gamma \delta}$ (painel inferior) e $\log (\mathrm{g})$ vs $\mathrm{D}_{0.2 \delta}$ (painel superior). Não há correlação entre os parâmetros comparados. . . .

3.18 Relação entre a intensidade da linha K do Ca II (3933.7 $\AA$ ) e a metalicidade estimada pelo SSPP. A relação mostra uma tendência coerente mesmo para estrelas com temperatura efetiva acima de $7500 \mathrm{~K}$, pois quanto menor a metalicidade, menor será a pseudo largura equivalente da linha do Cálcio. .

3.19 Limitação do método $\mathrm{f}_{m \delta}$ vs $\mathrm{D}_{0.2 \delta}$ para magnitude $\mathrm{g}>18$ (painéis esquerdos). A mesma dificuldade de separação entre estrelas anãs e gigantes ocorre para o método $c_{\beta \gamma \delta}$ vs $b_{\beta \gamma \delta}$ (painéis direitos), nos mesmos intervalos de magnitudes. 61

3.20 Limite de magnitude aparente para estrelas BHB da Galáxia. A proporção de estrelas no pico com $\log (\mathrm{g})$ típico de gigante começa a diminuir drasticamente para magnitude $g \sim 18 \ldots \ldots \ldots \ldots$

3.21 Distribuição de $\log (\mathrm{g})$ (para magnitudes $g>18$ ) de parâmetros gerados pelo SSPP. O pico centrado em $\log (\mathrm{g})=3.573(\sigma=0.328)$ representa a distribuição de estrelas gigantes e subgigantes e $\log (\mathrm{g})=4.274(\sigma=0.204)$ se aplica às estrelas anãs na sequência principal. . . . . . . . . . . . .

3.22 Parâmetros atmosféricos estelares gerados pelo SSPP para magnitude $g>$ 18. A incerteza na temperatura efetiva dada pelo SSPP é $\sigma_{T_{e f}}=157 \mathrm{~K}$ e a distribuição de $\log (\mathrm{g})$ tem $\sigma_{\log (g)}=0.328$. Ao contrário do critério adotado para estrelas com magnitude $g<18$, poucos objetos se encotram na região acima de $3 \sigma_{\log (g)}$. Nesse caso, a confiança na seleção é avaliada a cada $\sigma_{\log (g)}$, como mostram as Tabelas 3.2 e 3.1. . . . . . . . . . . . . . .

3.23 Parâmetros $c_{\beta \gamma \delta}$ vs $b_{\beta \gamma \delta}$ para estrelas com magnitude $g<18$. A figura foi colorida de acordo com os limites de separação de estrelas anãs adotados para o método do SSPP: estrelas BHB em azul, BS em verde e estrelas de sequência principal mais frias (F, G e K) em vermelho. A linha preta representa o ajuste polinomial de quarta ordem e a linha vermelha sua margem de erro $(3 \sigma=1.07) \ldots \ldots \ldots \ldots$ 
3.24 Resíduos do ajuste polinomial de ordem 4 sobre a região BHB $(g<18)$. $\langle\mathrm{x}\rangle$ $=-0.028$ e $\sigma=0.345 \ldots \ldots \ldots \ldots \ldots \ldots \ldots \ldots \ldots \ldots \ldots \ldots \ldots \ldots \ldots$

3.25 Estrelas que foram classificadas como anãs por todos os métodos. Os pontos estão coloridos segundo a margem de erro de temperatura efetiva $\left(\sigma_{T_{\text {ef }}}=\right.$ $157 \mathrm{~K}$ ). As estrelas com $\mathrm{T}_{e f}<7657 \mathrm{~K}$ são identificadas por pontos pretos, em laranja estão os objetos entre $7657 \mathrm{~K}<\mathrm{T}_{\text {ef }}<7814 \mathrm{~K}$ e em verde as estrelas com $\mathrm{T}_{e f}>7814 \mathrm{~K}$, que são as melhores candidatas a estrelas BS, destacadas dentro do retângulo. . . . . . . . . . . . . . . . . .

4.1 Diagrama cor-magnitude construído com o índice de cor do SDSS e a estimativa de magnitude de Kinman et al. (1994). Os pontos em vermelhos representam as estrelas com $[\mathrm{Fe} / \mathrm{H}]<-2.0$ e em azul com $[\mathrm{Fe} / \mathrm{H}]>-2.0$.

4.2 Diagrama cor-magnitude construído com o índice de cor $(B-V)$ e a estimativa de magnitude absoluta de Deason et al. (2011). Os pontos vermelhos representam estrelas com $[\mathrm{Fe} / \mathrm{H}]<-2.0$ e em azul com $[\mathrm{Fe} / \mathrm{H}]>-2.0$. . .

4.3 Comparação entre as distâncias estimadas pelo método de Kinman et al. (1994) e Deason et al. (2011). Eles adotam como referência, respectivamente, estrelas BS em aglomerados globulares e na corrente de Sagitário. As distâncias estimadas através da magnitude $g$ são sistematicamente menores pois desconsideram a dependência de $[\mathrm{Fe} / \mathrm{H}] \ldots \ldots \ldots$. . . . . . . .

4.4 Magnitudes absolutas na banda $V$ vs $[\mathrm{Fe} / \mathrm{H}]$ para as estrelas BS selecionadas. Assim como mostrou a Figura 1.4, a linha tracejada caracteriza o limite teórico para as estrelas BS em aglomerados globulares (através do ponto de turnoff da sequência principal), enquanto a linha contínua representa o mesmo limite considerando-se dados observacionais. . . . . . . . . . . . .

4.5 Distribuição de distâncias para estrelas BS e BHB. A linha em azul mostra a região onde a probabilidade de encontrar uma estrela BHB é maior que $70 \%$, a mesma probabilidade é vista para a linha vermelha sombreada, com estrelas BS. Figura retirada de Deason et al. (2011) . . . . . . . . . . . . .

4.6 Distribuição de distâncias para as estrelas BS estimadas através das Equações 4.3 e 4.5. Nota-se a coerência entre esta distribuição e a distribuições encontrada por Deason et al. (2011), apresentada na Figura 4.5. . . . . . . 
4.7 Distribuição das estrelas BS observadas na Galáxia em coordenadas galácticas XYZ. O plano XY contém o disco galáctico e Z define a distância até ele. Partindo do centro galáctico, a coordenada X aumenta no sentido do anticentro galáctico e Y aumenta no sentido de rotação da Galáxia. . . . . . . .

4.8 Relação teórica entre massa e tempo de vida na sequência principal em comparação ao Sol. As linhas verticais tracejadas mostram o intervalo de massa típico para estrelas A (com temperaturas efetivas equivalentes às estrelas BS selecionadas) e seu tempo de vida, identificados com linhas horizontais, para os casos onde $\mathrm{M}>2 \mathrm{M}_{\odot}\left(\right.$ em azul) e $\mathrm{M}<2 \mathrm{M}_{\odot}$ (em preto). Elas se distribuem entre 1.1 e 2.4 bilhões de anos. . . . . . . . . . . . . .

4.9 Na parte superior encontra-se o diagrama magnitude-cor das estrelas BS. É difícil distringuir, a partir de suas magnitudes, populações estelares diferentes ou superpostas, como mostra o painel inferior. . . . . . . . . .

4.10 Movimento próprio total em função da distância D. Nota-se que os movimentos próprios são grandes demais para as distâncias estimadas, e também, na maior parte dos dados, os erros médios (linha vermelha) são maiores que o valores do movimento próprio. . . . . . . . . . . . . . .

4.11 Velocidades U, V e W calculadas a partir dos movimentos próprios em função da metalicidade. Os painéis esquerdos mostram os valores obtidos para a toda a amostra e a direita vemos somente os objetos que possuem movimento próprio maior que seu erro. Em ambos os casos existem muitas estrelas que possuem velocidades superestimadas. . . . . . . . . . .

4.12 Fração estelar em função de $|\mathrm{Z}|$. O disco espesso é representado pela linha com pontos pretos, o halo interno em verde e o halo externo em vermelho. As frações não dependem da metalicidade. Figura retirada de Carollo et al.

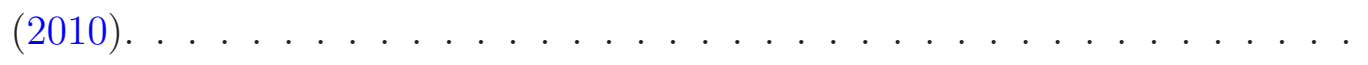

4.13 Distribuição das velocidades radiais heliocêntricas fornecidas pelo SSPP. . . 
4.14 Distribuições de $[\mathrm{Fe} / \mathrm{H}]$ em função da distância ao plano galáctico. As setas em azul identificam a metalicidade típica de cada componente galáctico: disco espesso $-[\mathrm{Fe} / \mathrm{H}]=-0.6$; disco espesso pobre em metais $-[\mathrm{Fe} / \mathrm{H}]=$ -1.3 ; halo interno $-[\mathrm{Fe} / \mathrm{H}]=-1.6$; halo externo: $[\mathrm{Fe} / \mathrm{H}]=-2.2$. As distribuições de metalicidade concordam com Carollo et al. (2010) até o halo interno. . . . . . . . . . . . . . . . . . . . . .

4.15 Erros dos parâmetros estelares $\left([\mathrm{Fe} / \mathrm{H}], \mathrm{T}_{e f}, \log (\mathrm{g})\right.$ e $\left.V_{\mathrm{R}_{\odot}}\right)$ em função da relação $\langle\mathrm{S} / \mathrm{R}\rangle$. O erro aumenta com a magnitude (pontos vermelhos: $g>$ 18) e consequentemente, para a maioria das estrelas do halo externo (pontos roxos: $|\mathrm{Z}|>17 \mathrm{kpc}$ ). A presença de pequenas populações com objetos de velocidades radiais destoantes não foi avaliada. Para tanto, é necessário reduzir a amostra de acordo com a precisão de interesse. . . . . . . . . .

4.16 Distribuição das velocidades radiais heliocêntricas para fornecidas pelo SSPP. Os painéis superiores mostram as distribuições para o halo interno e painéis inferiores as distribuições do halo externo. Há superposição de grupos com diferentes velocidades radiais no halo externo. . . . . . . . . . . . .

4.17 Distribuição das velocidades radiais heliocêntricas no halo externo. O painel superior esquerdo mostra a distribuição de $V_{R_{\odot}}$ para $|\mathrm{Z}|>17 \mathrm{kpc}$. Na parte inferior esquerda encontra-se a distribuição das estrelas BS em coordenadas galácticas. O retângulo destaca as estrelas com $\mathrm{Z}<-17 \mathrm{kpc}$, cuja distribuição de $V_{R_{\odot}}$ é vista no painel inferior direito. A distribuição de $V_{R_{\odot}}$ (painel superior direito) não apresenta duas populações superpostas para Z

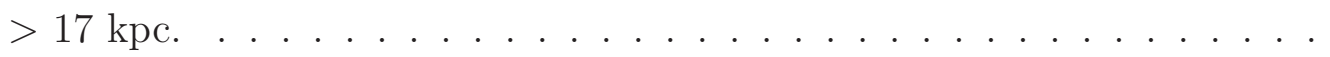


4.18 Distribuição das velocidades radiais galactocêntricas. Os painéis superiores mostram a distribuição de $V_{\mathrm{PGR}}$ para a amostra, os painéis centrais para $|\mathrm{Z}|<5 \mathrm{kpc}$ (disco espesso) e os inferiores, as distribuição para $|\mathrm{Z}|>5 \mathrm{kpc}$ (halo), a qual aparenta ser composta por ao menos duas populações. Os ajustes (à direita) mostram que as estrelas BS encontradas no disco espesso possuem distribuição simples em torno de zero, entretanto, a distribuição do halo não é simples, e portanto não pode ser representada por uma função gaussiana simples. A linha tracejada representa que nenhuma função foi ajustada nesse caso. . . . . . . . . . . . . . . . . . . . 87

4.19 Os painéis à esquerda mostram como varia a distribuição de $V_{\mathrm{PGR}}$ para as estrelas do halo ( $|\mathrm{Z}|>5 \mathrm{kpc}$ ) em função da distância galactocêntrica (R). Os painéis à direita mostram a variação da distribuição em função do módulo da distância ao plano galáctico $(|\mathrm{Z}|)$. Em ambos os casos, existe uma população de estrelas BS em torno de $V_{\mathrm{PGR}}=-150 \mathrm{~km} / \mathrm{s}$ (identificada com uma seta azul) que se torna cada vez mais evidente para $R$ e $|Z|$ maiores. 88

4.20 Velocidades galactocêntricas de estrelas BS e BHB da corrente de Sagitário no hemisfério Sul galáctico. Figura retirada de Koposov et al. (2012). . . .

4.21 Destaque da corrente de Sagitário vista através de estrelas BS com $|\mathrm{Z}|>$ $15 \mathrm{kpc}$. As estrelas destacadas em azul possuem velocidades radiais galactocêntricas entre $-180<V_{\mathrm{PGR}}(\mathrm{km} / \mathrm{s})<-50$. Muitas delas estão localizadas na região da corrente de Sagitário, evidenciada com retângulos pretos. 89

4.22 Corrente de Sagitário identificada por contraste de estrelas de sequência principal no ponto de turnoff com $0<(g-i)<0.7$ e $19.5<i<22.0$ (SDSS-DR8). A localização da galáxia anã remanescente da corrente de Sagitário é identicada pela estrela vermelha. A linha tracejada em vermelho marca a projeção do plano orbital da galáxia anã (consequentemente a posição da corrente). Figura retirada de Koposov et al. (2012). . . . . . . . 
4.23 Distâncias das estrelas BS identificadas com altas velocidades radiais galactocêntricas negativas presentes nas regiões da corrente de Sagitário (vistas na Figura 4.21). A linha preta indica a distância até a corrente $\left(\mathrm{D}_{S g}=\right.$ $26.1 \pm 5.6 \mathrm{kpc}$ ) calculada por Watkins et al. (2009) e a linha azul a distância estimada por este trabalho $\left(\mathrm{D}_{B S}=24.74 \pm 5.37 \mathrm{kpc}\right) . \ldots . \ldots . . . . \quad 90$

B.1 Função gaussiana com $\mathrm{A}=\mathrm{x}_{0}=\sigma=1$. A cada desvio padrão, são vistas as probabilidades de se observar a variável aleatória. . . . . . . . . . . . . 113 


\section{Lista de Tabelas}

1.1 Linhas da série de Balmer do Hidrogônio. . . . . . . . . . . . . . . . . . . . 34

3.1 Confiabilidade na seleção da amostra de estrelas BS (expressa em \%). . . . 63

3.2 Número de estrelas presentes em cada intervalo de erro da Tabela 3.1. . . . 63

A.1 Parâmetros do ajuste multipico com duas gaussianas (Equação B.3) - Figura

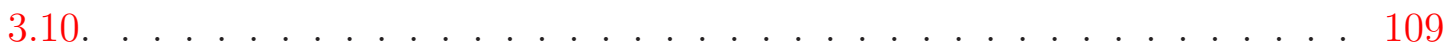

A.2 Parâmetros do ajuste multipico com duas gaussianas (Equação B.3) - Figura

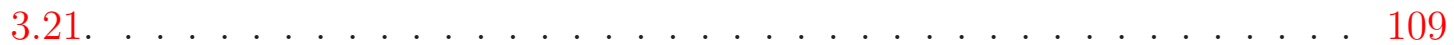

A.3 Parâmetros do ajuste do polinômio de quarto grau visto na Figura 3.23 $\mathrm{f}(\mathrm{x})=\mathrm{a} x^{4}+\mathrm{b} x^{3}+\mathrm{c} x^{2}+\mathrm{d} x+\mathrm{e} \ldots \ldots \ldots \ldots . \ldots 110$

A.4 Parâmetros do ajuste dos resíduos (Equação B.3) - Figura 3.24. . . . . . . 110

B.1 Probabilidade (P) de $x$ ser encontrado em torno de seu valor médio (em \%). 112 



\section{Sumário}

1. Introdução . . . . . . . . . . . . . . . . . . . . . . . . . . 27

1.1 Possíveis origens das estrelas azuis tardias . . . . . . . . . . . . . 27

1.2 Estrelas BS em aglomerados abertos . . . . . . . . . . . . . . . . 29

1.3 Estrelas BS em aglomerados globulares . . . . . . . . . . . . . . . . 30

1.4 Estrelas BS no campo . . . . . . . . . . . . . . . . . . . . . . . . 32

1.4.1 O que é ser uma estrela "azul tardia" de campo? . . . . . . . . . . 34

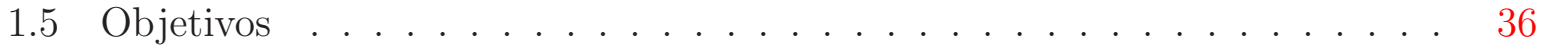

2. Base de Dados . . . . . . . . . . . . . . . . . . . . . . . . . . . . . 37

2.1 SDSS e o Data-release 8 . . . . . . . . . . . . . . . . . 37

2.2 Restrições no diagrama cor-cor . . . . . . . . . . . . . . . . . . . . . . . 38

2.3 Inspeção e qualidade dos espectros . . . . . . . . . . . . . . . . . . . 40

2.4 Parâmetros atmosféricos - SSPP . . . . . . . . . . . . . . . 40

2.5 As linhas da série de Balmer do Hidrogênio . . . . . . . . . . . . . . . . . 43

3. Seleção de Estrelas BS . . . . . . . . . . . . . . . . . . . . . . . . 45

3.1 Método $\mathrm{f}_{m}$ vs $\mathrm{D}_{0.2} \ldots \ldots \ldots \ldots \ldots \ldots \ldots \ldots$

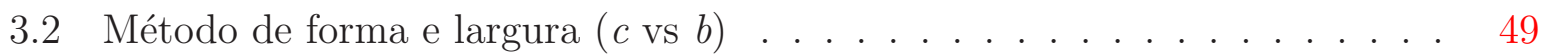

3.3 Método do SSPP . . . . . . . . . . . . . . . . . . . . 51

3.4 O SSPP para estrelas quentes . . . . . . . . . . . . . . . . 54

3.5 Estrelas com magnitude $g>18 \ldots \ldots \ldots$. . . . . . . . . . . 59

3.6 Combinação de métodos . . . . . . . . . . . . . . . . . . . . . . 61

3.7 Discussão . . . . . . . . . . . . . . . . . . . . . . . . . . . . . . . . . . . . 62 


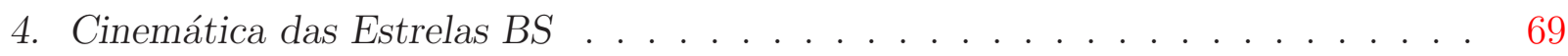

4.1 Estimativas de magnitude absoluta e distâncias . . . . . . . . . . . . . 69

4.1.1 Magnitude absoluta $M_{V} \ldots \ldots \ldots$. . . . . . . . 70

4.1.2 Magnitude absoluta $M_{g} \ldots \ldots \ldots . \ldots . \ldots . \ldots 71$

4.1.3 Tempo de vida na sequência principal . . . . . . . . . . . . . . . . 75

4.2 Distribuições de velocidades e a busca por correntes estelares . . . . . . . . 76

4.2.1 Componentes de velocidades $\mathrm{U}, \mathrm{V}$ e W . . . . . . . . . . . 77

4.2.2 Velocidades radiais heliocêntricas . . . . . . . . . . . . . . 79

4.2.3 Velocidades radiais galactocêntricas . . . . . . . . . . . . 86

4.3 Discussão . . . . . . . . . . . . . . . . . . . . . . . . . . 89

5. Conclusões e perspectivas . . . . . . . . . . . . . . . . . 93

5.1 Principais resultados . . . . . . . . . . . . . . . . . 93

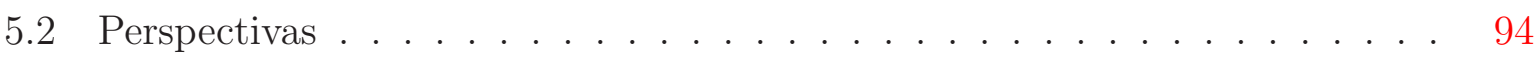

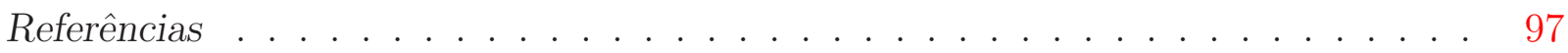

$\begin{array}{ll}\text { Apêndice } & 107\end{array}$

A. Ajustes . . . . . . . . . . . . . . . . . . . . 109

B. Histogramas, gaussianas e o tratamento estatístico . . . . . . . . . . . . 111

B.1 Histograma e a escolha da raiz quadrada . . . . . . . . . . . . . . . . . . 111

B.2 Gaussianas . . . . . . . . . . . . . . . . . . . . . 112

B.2.1 Regra empírica . . . . . . . . . . . . . . . . . . . 112 
Capítulo 1

\section{Introdução}

As estrelas "azuis tardias" (blue stragglers stars - estrelas BS) são estrelas de sequência principal que apresentam um aparente atraso evolutivo em relação à região onde se encontram, ou seja, parecem ser jovens e excepcionalmente quentes (e, portanto, mais massivas) que suas vizinhanças. Essas estrelas "azuis" foram observadas pela primeira vez por Sandage (1953), quando estudava a idade do aglomerado globular M3. Ele notou que algumas estrelas que se encontravam na sequência principal eram mais azuis que as estrelas do ponto de turnoff. Tal constatação possibilitou a caracterização dessa nova população estelar. Essa identificação é exemplificada na Figura 1.1, através do diagrama HR do aglomerado globular M55, onde as estrelas azuis tardias identificadas na sequência principal, aparecem dentro do trapézio.

\subsection{Possíveis origens das estrelas azuis tardias}

Desde sua descoberta, as estrelas BS têm sido identificadas em todo tipo de ambiente: em aglomerados globulares, aglomerados abertos (Mathieu e Latham, 1986), nas populações do campo (Preston e Sneden, 2000; Carney et al., 2005) e até em galáxias anãs do grupo local (Momany et al., 2007).

Ainda não é claro como as estrelas BS se formam. Muitas origens têm sido propostas para explicar a existência delas e, consequentemente, justificar seu aparente atraso evolutivo. Leonard (1989) e Stryker (1993) fizeram resumos cronológicos sobre as hipóteses levantadas desde a década de 60, os quais são acrescidos de considerações atuais abaixo:

(1) Estrelas BS são estrelas que nasceram depois da maioria das estrelas presentes em seu sistema (Roberts, 1960). Tal processo pode ocorrer para aglomerados jovens, mas não para 


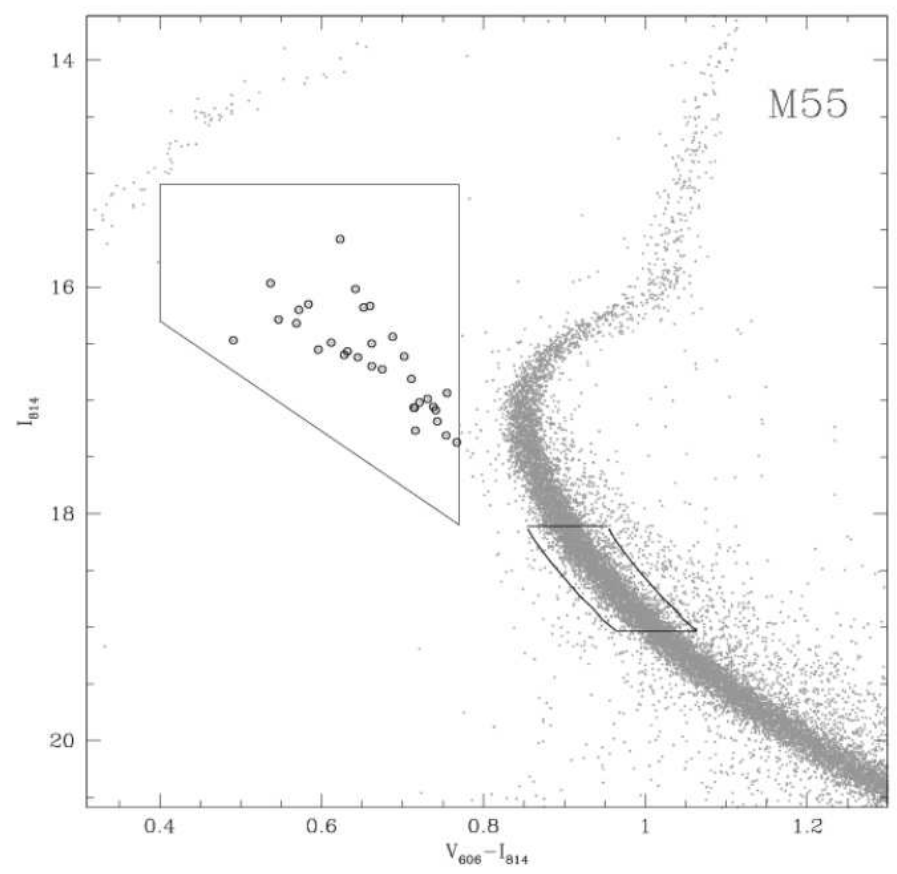

Figura 1.1: Diagrama HR do aglomerado globular M55. As estrelas azuis tardias (círculos abertos) são identificadas na sequência principal dentro do trapézio. O losango mostra estrelas na sequência principal, antes do ponto de turnoff. Vê-se claramente que as estrelas BS localizam-se no prolongamento da sequência principal. Figura retirada de Sollima et al. (2008).

aglomerados globulares, cujo gás já foi consumido. Além disso não há acresção de gás do meio interestelar nesses sistemas.

(2) Estrelas BS são estrelas que ganharam massa via acresção de gás do meio interestelar ou do meio intra-aglomerado (Williams, 1964). Essa hipótese é pouco provável pois a acresção de gás do meio interestelar por uma estrela isolada não ocorre.

(3) Estrelas BS são estrelas em sistemas binários cerrados que aumentaram suas massas devido à transferência de gás por suas companheiras (McCrea, 1964). Posteriormente, Zinn e Searle (1976) afirmaram que alguns sistemas binários podem, não somente transferir massa, como também coalescer completamente. Essa hipótese foi verificada por Iben (1986), cujo trabalho mostrou que existem estrelas BS geradas por transferência de massa. (4) Estrelas BS são estrelas evoluídas que retornaram para a sequência principal (Sargent, 1968). Essa explicação é improvável se se considera razões dinâmicas (Mathieu e Latham, 
1986; Nemec e Harris, 1987; Nemec e Cohen, 1989). Além disso, não são observadas estrelas no ramo horizontal retornando até a sequência principal.

(5) Estrelas BS são estrelas que se fundiram através de colisões físicas em aglomerados globulares (Hills e Day, 1976). A alta densidade estelar encontrada no núcleo dos aglomerados globulares indica que estas colisões podem ocorrer, e que o resultado da colisão entre duas estrelas na sequência principal seria uma estrela massiva e "bem-misturada" (Benz e Hills, 1987). Esse processo tem sido identificado no núcleo de aglomerados globulares densos.

(6) Estrelas BS são estrelas isoladas com tempos de vida estendidos devido à eficiente mistura interna de elementos (Wheeler, 1979). A mistura pode ser o resultado de uma rápida rotação ou um campo magnético muito forte. Essa hipótese recebeu suporte de trabalhos na década de 80 (Abt, 1985; Schild e Berthet, 1986; Maeder, 1987; Mathys, 1987)), mas ainda não houve comprovação observacional.

(7) Estrelas BS são estrelas jovens que foram capturadas por aglomerados estelares (Leonard, 1985). Esse processo pode explicar algumas estrelas BS presentes em aglomerados abertos, e provavelmente nenhuma estrela BS em aglomerados globulares. Simulações feitas em sistemas de N-corpos mostraram que a captura de estrelas isoladas por aglomerados é muito improvável quando são adotadas velocidades usuais das estrelas na Galáxia (Leonard, $1985)$.

(8) Estrelas BS são estrelas de sequência principal que têm "evitado" perder massa (Willson et al., 1987). Esse cenário radical de evolução estelar afirma que as estrelas de sequência principal são normalmente instáveis durante suas vidas, pulsando e rotacionando rapidamente. Como consequência, perdem massa gradativamente. Assim, elas desceriam a sequência principal do diagrama HR. Entretanto, estrelas com baixa rotação não sofrem essa perda de massa elevada e aparecem como estrelas BS.

\subsection{Estrelas BS em aglomerados abertos}

As hipóteses (1) e (7) aplicam-se fundamentalmente a aglomerados abertos e, juntamente com a hipótese (3), são os processos capazes de explicar as estrelas azuis tardias nesses ambientes atualmente. Dentre os vários cenários onde as estrelas BS são encontradas, os aglomerados abertos são aqueles onde as explicações têm o menor consenso (Lu 
et al., 2011). As estrelas azuis tardias nesses ambientes devem ser aparentemente mais jovens do que o próprio aglomerado, ou seja, devem ser estrelas muito massivas (estando entre os tipos espectrais $\mathrm{O}$ e B). É difícil ajustar a sequência principal para a região das estrelas massivas dada sua baixa representatividade numérica. Assim, as dificuldades em explicar a classe de estrelas BS em aglomerados abertos começam por sua própria identificação e classificação.

Devido às numerosas origens que têm sido propostas, cada tentativa de se confirmar ou excluir uma hipótese deve ser explorada. A densidade de estrelas encontrada nos aglomerados abertos não favorece a origem colisional. Além disso, a hipótese rotacional também foi excluída, pois a rotação das estrelas BS é insuficiente para explicar as enormes diferenças de cores encontradas entre elas e as demais estrelas da sequência principal (Mermilliod, 1982). Recentemente, Lu et al. (2011) mostraram que a transferência de massa em sistemas binários parece ser a melhor alternativa, sendo que sistemas binários que possuem estrelas em diferentes estágios evolutivos podem produzir estrelas BS de diferentes temperaturas.

\subsection{Estrelas BS em aglomerados globulares}

Nos aglomerados globulares, as hipóteses (3) e (5) parecem ser suficientes para justificar a existência das BSs nesses ambientes. Elas são observadas em aglomerados velhos como estrelas de sequência principal de tipo espectral A e F.

As principais discussões sobre suas origens nesses ambientes modelam a fração de contribuição predominante de cada processo para cada aglomerado (Fusi Pecci et al., 1992): no núcleo do aglomerado a contribuição colisional (devido à alta densidade de estrelas) é predominante, e na periferia do aglomerado, a fração de estrelas BS formadas por transferência de massa é significativa (Ferraro et al., 1997; Mapelli et al., 2004, 2006). Esse cenário tem favorecido o modelo de transferência de massa devido ao crescente número de estrelas BS encontradas em regiões periféricas de aglomerados globulares. Nesses ambientes, sistemas binários tem maior probabilidade de sobreviver. Mapelli et al. (2004) conseguiram reproduzir a população de estrelas BS no aglomerado globular 47 Tuc usando uma combinação de criação de estrelas BS por colisão e transferência de massa (Figura $1.2)$.

A ferramenta mais utilizada para distinguir os processos que formam estrelas azuis 


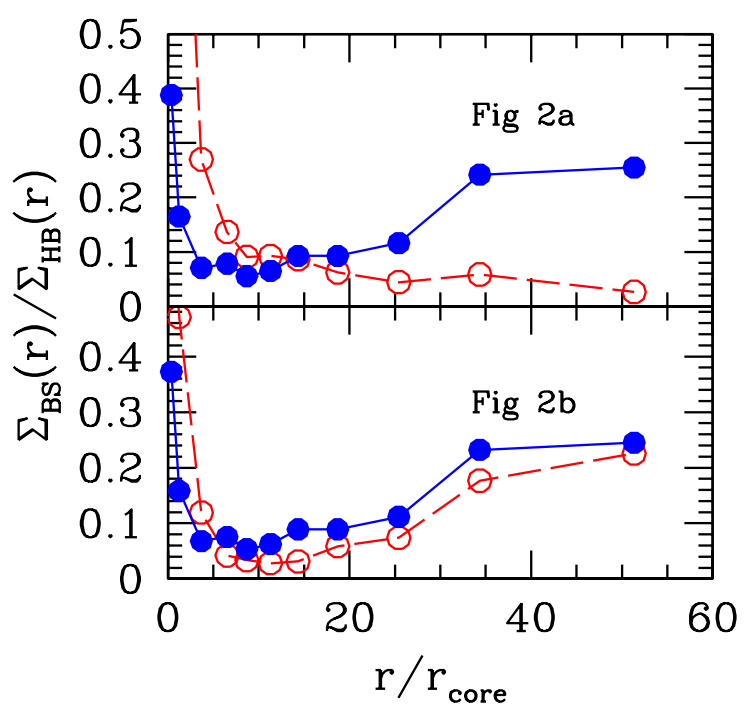

Figura 1.2: Comparação entre a densidade superficial de estrelas BS observada (linha azul) e a densidade superficial de estrelas BS simulada (linha vermelha) para o algomerado globular 47 Tuc. Na parte superior vê-se a simulação somente com contribuição do processo de formação de estrelas BS por colisões e, na parte inferior, a simulação com processo colisional e com tranferência de massa de sistemas binários. Ambas as distribuições são normalizadas: no eixo x com respeito ao raio do núcleo do aglomerado $\left(\mathrm{r}_{\text {core }}\right)$ e no eixo y em relação ao número de estrelas de ramo horizontal (horizontal branch - HB) catalogadas por Ferraro et al. (2004). Figura retirada de Mapelli et al. (2004).

tardias em aglomerados globulares é a espectroscopia em alta resolução. Abundâncias químicas anômalas são esperadas na superfície de estrelas BS formadas por transferência de massa (Sarna e De Greve, 1996), principalmente de elementos do tipo CNO, enquanto tais discrepâncias não são preditas para estrelas BS colisionais (Lombardi et al., 1995). Contudo, estudos em alta resolução para verificar esses fenômenos ainda são recentes e levam em conta apenas algumas estrelas BS em 47 Tuc, como mostra a Figura 1.3 (Ferraro et al., 2006), onde a abundância $[\mathrm{O} / \mathrm{Fe}]^{1}$ é comparada com a carbonicidade $[\mathrm{C} / \mathrm{Fe}]$.

\footnotetext{
${ }^{1}$ A abundânca de um elemento em relação a outro é geralmente indicada através da comparação de suas razões numéricas relativas ao Sol, utilizando-se a notação: $[\mathrm{A} / \mathrm{B}]=\log \left(N_{A} / N_{B}\right)_{\star}-\log \left(N_{A} / N_{B}\right)_{\odot}$, onde $N_{A}$ e $N_{B}$ referem-se aos números de átomos dos elementos A e B na estrela $(\star)$ e no Sol $(\odot)$.
} 


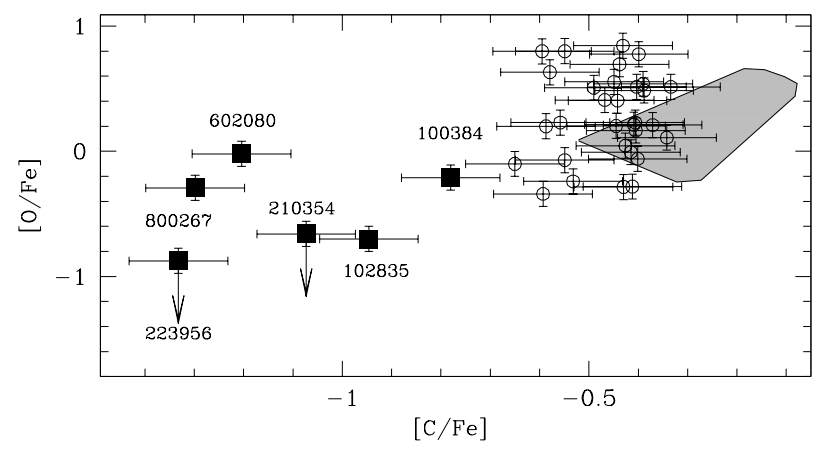

Figura 1.3: Razão $[\mathrm{O} / \mathrm{Fe}]$ em função de $[\mathrm{C} / \mathrm{Fe}]$ para as estrelas azuis tardias observadas no aglomerado globular 47 Tuc. Estrelas BS "normais" são marcadas com círculos vazios, enquanto que 6 estrelas BS com menos Carbono e Oxigênio são marcadas com quadrados. Seus nomes estão indicados. A região cinza corresponde ao local de outras estrelas do ponto de turnoff do aglomerado 47 Tuc. Figura retirada de Ferraro et al. (2006).

\subsection{Estrelas BS no campo}

Dentre a variedade de explicações propostas que têm como base a evolução estelar, a transferência de massa não requer alterações nas teorias, enquanto o mesmo não ocorre em relação a perda de massa por pulsações (Willson et al., 1987) e mistura interna eficiente (Wheeler, 1979). Entretanto, a verificação dessas hipóteses ainda é limitada, razão pela qual o entendimento do fenômeno têm sido abordado, preferencialmente, através das hipóteses mensuráveis.

Preston e Sneden (2000) expandiram o trabalho anterior de Preston et al. (1994) e argumentaram que as estrelas BS encontradas no campo foram criadas, quase que unicamente, pelo processo de transferência de massa. Recentemente, os estudos de Carney et al. (2001) e Carney et al. (2005) corroboraram com essa proposta. Tal afirmação, não somente possui senso qualitativo, como também foi sustentada pela identificação de estrelas BS no campo, em frequência maior do que em regiões de baixa densidade em aglomerados globulares (Preston et al., 1994). Com isso, Preston e Sneden (2000) concluiram ainda que, mesmo em regiões de baixa densidade em aglomerados, sistemas binários podem ser destruídos, diminuindo o número de estrelas BS observadas.

Quando se trata de aglomerados, deve-se ter em mente que mesmo as estrelas encontradas em regiões periféricas de baixa densidade podem ser vulneráveis a encontros estelares, pois estes sistemas podem ser carregados para a região central do aglomerado, onde a 
densidade estelar é maior. Portanto, as estrelas BS de campo fornecem uma amostra para o estudo do mecanismo que pode ter criado tais "estranhos" objetos através da evolução estelar, independentemente do ambiente onde elas se encontram.

Os estudos de Preston e Sneden (2000) consistiram em um levantamento de 8 anos de observações de 62 estrelas azuis pobres em metais, cujo objetivo principal era identificar variações em suas velocidades radiais. Uma vez que as colisões estelares são pouco frequentes no disco e no halo, as estrelas BS de campo identificadas (Preston et al., 1994) deveriam ser criadas unicamente pelo processo de transferência de massa (McCrea, 1964). Dos 62 objetos, $70 \%$ são sistemas binários espectroscópicos com período orbital entre $2<$ $\mathrm{P}_{\text {orbital }}($ dias $)<4000$. A análise química desses objetos indicou que ao menos metade deles são estrelas de sequência principal inferior acrescidas de massa por suas companheiras, enquanto estas atravessavam a evolução pós-sequência principal. Suas companheiras invisíveis são, provavelmente, anãs brancas. Dos 30\% restantes (não binários), cerca de 1/3 pode ser representado por sistemas que coalesceram (Vilhu, 1982), devido a proximidade das estrelas presentes no sistema (período orbital de $\sim 5$ dias). Os autores argumentaram que o restante da amostra pode ser representado por estrelas azuis pobres em metais de idade intermediária, as quais teriam sido capturadas de galáxias anãs satélites, como a galáxia esferoidal anã Carina (Smecker-Hane et al., 1994), já que não existem evidências de que a Galáxia preserve nuvens de gás de baixa metalicidade. A mesma solução já havia sido apontada por Preston et al. (1994), que consideraram a origem das estrelas BS como resultado de uma possível interação da Galáxia com uma galáxia anã entre 3 e 10 bilhões de anos atrás. Entretanto, nada impede que esses sistemas simples sejam estrelas BS resultantes da coalescência de ex-sistemas binários muito próximos, ou simplesmente sistemas binários que não se apresentem na linha de visada. Assim, 20\% das estrelas BS identificadas no campo podem apresentar velocidades radiais com comportamento diferente das estrelas BS formadas por transferência de massa na Galáxia ( 80\%).

Além disso, Preston e Sneden (2000) verificaram que a porcentagem de binárias não depende da metalicidade, independente de se tratar de estrelas do disco espesso ou do halo. O monitoramento da velocidade radial para estrelas binárias de longo período é naturalmente lento, demandando vários anos de observação. Logo, não fornecem estatística suficiente para analisar o fenômeno BS em maior escala. 
Por outro lado, os novos surveys tem reunido grande quantidade de objetos, em especial, estrelas de tipo espectral A. Dentre as estrelas do tipo A estão várias candidatas a estrelas BS. Portanto, a seleção desses objetos nas novas bases de dados, juntamente com a determinação de seus parâmetros físicos e químicos, podem fornecer pistas sobre a natureza do fenômeno BS em grande escala, através da análise de padrões de metalicidade, distâncias e velocidades radiais.

\subsubsection{O que é ser uma estrela "azul tardia" de campo?}

Considera-se que uma estrela BS de campo seja uma estrela de sequência principal, pobre em metal e que seja mais quente que a média dos pontos de turnoff da sequência principal encontrados em aglomerados globulares (Figura 1.4). Em suma, são estrelas de sequência principal de tipo espectral A pobres em metais que se encaixam satisfatoriamente nesse perfil.

Elas são particularmente interessantes para essa caracterização pois possuem temperaturas efetivas entre $7500 \mathrm{~K}$ e $10000 \mathrm{~K}$, massas entre $1.6 \mathrm{M}_{\odot}$ e $2.2 \mathrm{M}_{\odot}$ e espectros caracterizados por linhas da série de Balmer do Hidrogênio (Tabela 1.1) muito evidentes (Habets e Heintze, 1981; Adelman, 2004). Assim, são estrelas brilhantes que possuem espectros característicos que se distiguem de outras estrelas, mesmo em baixa resolução espectral $(\sim 1 \AA)$. Seu tempo de vida na sequência principal está estimado entre 1.1 bilhão de anos (para as estrelas com $\sim 9600 \mathrm{~K}$ ) até 2.5 bilhões de anos (para estrelas com $\sim 7500 \mathrm{~K})($ Adelman, 2004). São estrelas frequentes no disco, onde a população estelar é jovem. Mas, são ovelhas negras no halo, onde a população de estrelas é velha (entre 10 e 12 bilhões de anos), segundo Jofré e Weiss (2011). Os exemplares mais brilhantes e próximos dessas estrelas são Sirius A, Vega e Altair.

Tabela 1.1 - Linhas da série de Balmer do Hidrogônio.

\begin{tabular}{|c|c|c|c|c|c|c|c|c|}
\hline Transição & $3 \rightarrow 2$ & $4 \rightarrow 2$ & $5 \rightarrow 2$ & $6 \rightarrow 2$ & $7 \rightarrow 2$ & $8 \rightarrow 2$ & $9 \rightarrow 2$ & $\infty \rightarrow 2$ \\
\hline Nome & $\mathrm{H}_{\alpha}$ & $\mathrm{H}_{\beta}$ & $\mathrm{H}_{\gamma}$ & $\mathrm{H}_{\delta}$ & $\mathrm{H}_{\epsilon}$ & $\mathrm{H}_{\zeta}$ & $\mathrm{H}_{\eta}$ & - \\
\hline$\lambda(\AA)$ & 6563 & 4861 & 4341 & 4102 & 3970 & 3889 & 3835 & 3646 \\
\hline
\end{tabular}

Além do tempo de vida na sequência principal, outra relação para a idade das estrelas é fornecida através da abundânca de ferro (metalicidade $-[\mathrm{Fe} / \mathrm{H}]$ ). As estrelas transformam 


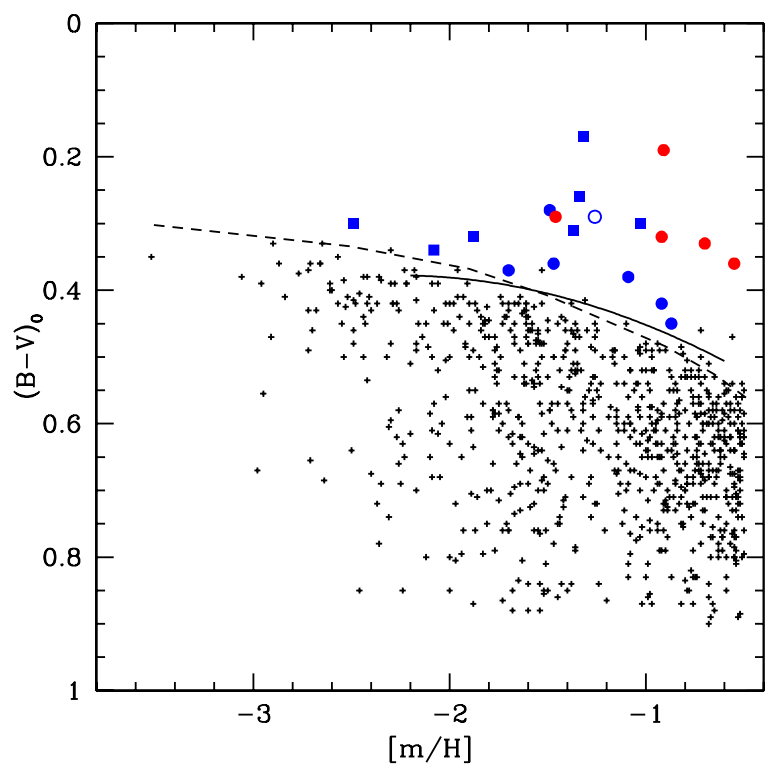

Figura 1.4: Relação entre o índice de cor desavermelhado $(\mathrm{B}-\mathrm{V})_{0}$ vs metalidade para estrelas de sequência principal pobres em metais do campo (cruzes pretas) e, para 5 estrelas BS encontradas por Carney et al. (2005) (círculos vermelhos). Também são mostradas 6 estrelas BS binárias do trabalho de Carney et al. (2001) (círculos azuis), além de 7 estrelas BS que apresentaram solução orbital binária com período maior que 20 dias no trabalho de Preston e Sneden (2000) (quadrados preenchidos em azul).A estrela BD+25 1981, que pode ser binária, é graficada como um círculo azul aberto. A linha sólida representa um ajuste às regiões de cor onde se encontram os pontos de turnoff da sequência principal de aglomerados globulares, e a linha tracejada identifica o seu equivalente teórico. Figura retirada de Carney et al. (2005).

continuamente os elementos químicos em seus interiores; são elas as responsáveis pelo enriquecimento químico do Universo após o Big-Bang. Em vista da infinidade de linhas de absorção que surgem a partir de transições associadas com átomos de ferro no Sol, Fe tem sido tradicionalmente utilizado como elemento referência para comparar a metalicidade de uma estrela com outra, quantificada como $[\mathrm{Fe} / \mathrm{H}]$. As chamadas estrelas pobres em metais contêm quantidades muito pequenas de elementos pesados (metais), diferentes de H e He. Elas foram formadas antes do Universo ser significantemente enriquecido em elementos químicos como é hoje. Portanto, as estrelas mais velhas da Galáxia são fósseis estelares e possibilitam a reconstrução da evolução química da Galáxia, desde os primórdios até os dias atuais (Beers e Christlieb, 2005). 


\subsection{Objetivos}

A identificação de estrelas tipo A pobres em metais de campo suscita interessantes questões: como podem existir estrelas, aparentemente jovens por serem azuis? como elas são formadas? qual é a sua distribuição de velocidades? como se comportam cinematicamente em relação aos halos interno e externo da Galáxia?

Este projeto visa fornecer mais subsídios para respostas a algumas das questões anteriores. Para tanto, utiliza-se os dados espectrofotométricos do levantamento digital do céu SDSS (Sloan Digital Sky Survey), assim como os seus parâmetros atmosféricos obtidos através de seu sistema de análise automatizado (Segue Stellar Parameter Pipeline - SSPP).

Dentre os espectros estelares, busca-se a separação e classificação daqueles que possuam caracteríscticas físicas de estrelas azuis tardias no campo galáctico: estrelas de tipo espectral A (pobres em metais) que estejam na sequência principal. Isso permitirá estabelecer uma visão ampla dessa população estelar pouco compreendida, além de fornecer uma nova base para futuras investigações, tais como monitoramento de velocidades radiais para comprovação ou não de binariedade e/ou abundância química em alta resolução para comprovação do enriquecimento químico via acresção. A base de dados de estrelas BS no campo permitirá também fazer correlações do fenômeno "azul tardio" que ocorre no campo, com o processo identificado em aglomerados ou em outras galáxias.

$\mathrm{Na}$ análise deste projeto é feita a comparação das duas principais teorias capazes de explicar as estrelas BS no campo: formação por sistemas binários galácticos que tiveram transferência de massa (ou coalesceram) e a origem extragaláctica. A origem extragaláctica considera que tais estrelas seriam prováveis membros de galáxias anãs (pobres em metais) que foram atraídas pela Galáxia. Para tanto, espera-se que as velocidades radiais das estrelas oriundas desses processos (segundo as predições, devem representar $\sim 20 \%$ da amostra) apresentem comportamentos diferentes entre suas distribuições de velocidades.

No Capítulo 2 são apresentados os dados utilizados ao longo do trabalho, bem como os critérios adotados em sua seleção. No Capítulo 3 são vistos os métodos de separação entre estrelas do tipo A na sequência principal e de ramo horizontal. As estimativas de magnitude absoluta, distância e uma primeira abordagem cinemática adotada encontramse no Capítulo 4. Finalmente, no Capítulo 5 são apresentadas as principais conclusões e perspectivas do projeto. 
Capítulo 2

\section{Base de Dados}

Neste capítulo apresenta-se inicialmente a base de dados utilizada neste trabalho, os critérios iniciais de seleção de estrelas BS, através da região do diagrama cor-cor habitada preferencialmente por estrelas de tipo A. Em seguida, discute-se o limite de $[\mathrm{Fe} / \mathrm{H}]$ adotado para as estrelas pobres em metais da amostra. Por fim, mostra-se a essência do método que separa espectroscopicamente as estrelas de tipo A anãs das gigantes.

\subsection{SDSS e o Data-release 8}

O Sloan Digital Sky Survey (SDSS) é um grande mapeamento espectroscópico e fotométrico (York et al., 2000) que cobre cerca de 1/4 do céu. Os dados de imageamento são obtidos pela instrumentação de dois conjuntos de matrizes de CCDs (Tektronix) acoplados ao telescópio de 2.5m (Gunn et al., 1998, 2006) situado no Apache Point Observatory, Novo México - EUA.

O imageamento é feito simultaneamente em cinco bandas visíveis (ugriz: centradas em 3551, 4686, 6166, 7480 e $8932 \AA$, respectivamente, ver: Fukugita et al., 1996). Os dados são processados automaticamente através de diversos métodos fotométricos, astrométricos e espectrais, atualmente unificados e automatizados no que é conhecido como SSPP (Segue Stellar Parameter Pipeline) do SDSS (Lupton et al., 2001; Stoughton et al., 2002; Pier et al., 2003; Ivezić et al., 2004; Tucker et al., 2006; Lee et al., 2008; Allende Prieto et al., 2008).

Em janeiro de 2011, o SDSS publicou seu oitavo data-release (DR8) contendo todos os dados já obtidos pelo mapeamento em mais de $14000^{\circ 2}$ do hemisfério norte celeste (Aihara et al., 2011). A Figura 2.1 mostra a distribuição dos dados utilizados nesse trabalho, os quais estão presentes em todas as regiões do survey. 


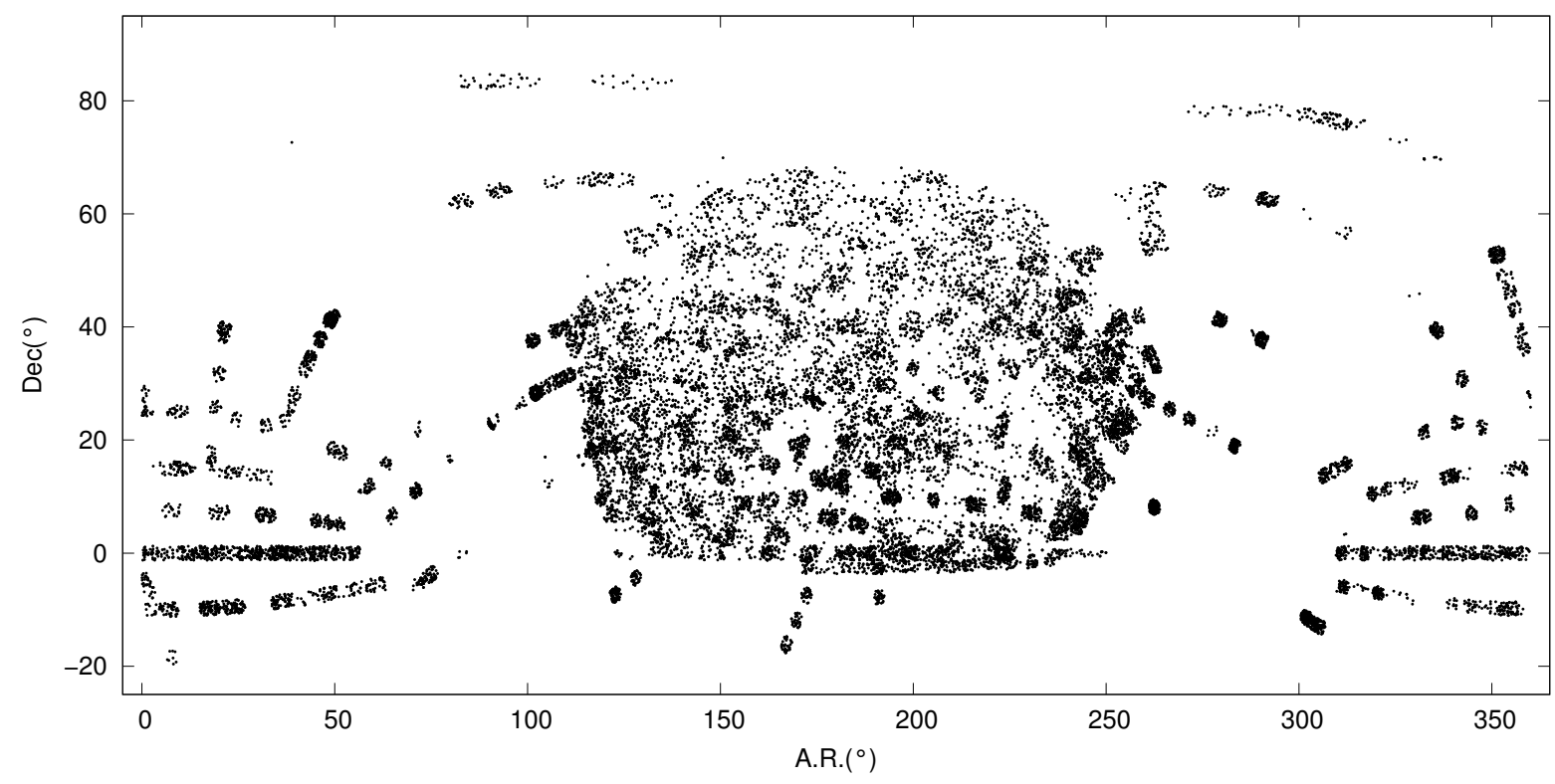

Figura 2.1: Coordenadas equatoriais das estrelas analisadas neste trabalho na região observada pelo telescópio do SDSS.

Nenhuma restrição em coordenadas foi aplicada, pois pretende-se selecionar estrelas BS tanto no disco como no halo da Galáxia. Além disso, ao longo do trabalho são adotados os mapas de extinção descritos por Schlegel et al. (1998). Assim, as magnitudes e índices de cor vistos a seguir já estão corrigidos por avermelhamento.

\subsection{Restrições no diagrama cor-cor}

No banco de dados do SDSS, as candidatas a estrelas azuis tardias são frequentemente encontradas na região do diagrama cor-cor destacado em vermelho na Figura 2.2, cujos limites são: $0.90<(u-g)<1.40$ e $-0.25<(g-r)<0.00$. A região é habitada preferencialmente por estrelas quentes do tipo espectral A. Entretanto, a fotometria não distingue estrelas de tipo A anãs (candidatas a estrelas BS) de estrelas gigantes azuis do ramo horizontal (blue horizontal branch stars - estrelas BHB). Nesse diagrama, as estrelas de tipo A se confundem até mesmo com estrelas mais frias de tipo espectral F, G e K.

A seleção fotométrica adotada neste trabalho segue os critérios tradicionais (ver, por exemplo: Yanny et al., 2000; Sirko et al., 2004; Xue et al., 2008; Deason et al., 2011) com intervalos ligeiramente maiores (ver Figura 2.3). Normalmente, os esforços dedicados ao 


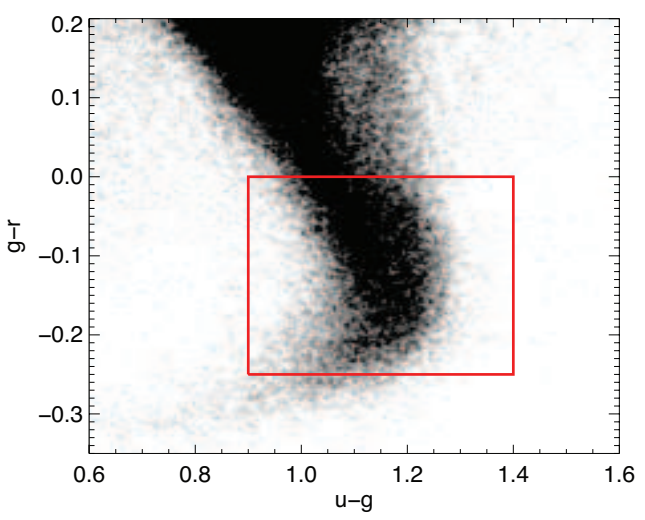

Figura 2.2: Dados do DR8 e sua distribuição no diagrama cor-cor, a região destacada em vermelho destaca onde são encontradas as estrelas do tipo A. Os limites dessa região são: $0.90<(u-g)<1.40$ e $-0.25<(g-r)<0.00$. Figura retirada de Deason et al. (2011).

estudo de estrelas do tipo A visam isolar as estrelas puramente BHB, objetos que não são alvo de estudo neste trabalho.

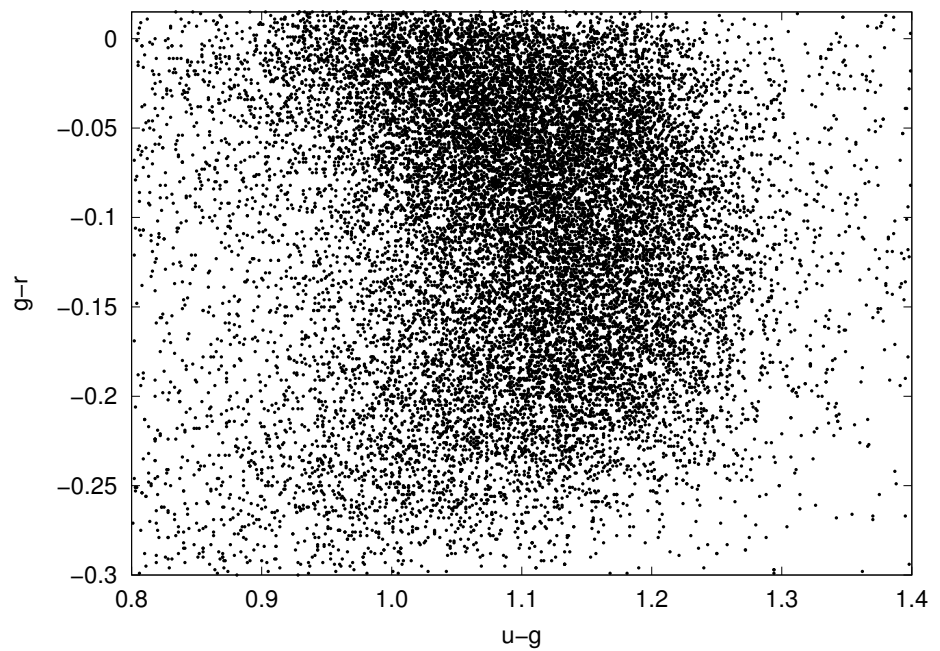

Figura 2.3: Região do diagrama cor-cor adotada para espectros do DR8: $0.80<(u-g)<$ 1.40 e $-0.300<(g-r)<0.015$.

Assim, ao invés de adotar o limite de Deason et al. (2011), a região considerada na Figura 2.3 foi estendida com o objetivo de incluir mais estrelas anãs. Os novos limites adotados são: $0.80<(u-g)<1.40$ e $-0.30<(g-r)<0.015$, pois é justamente na região $(g-r)>0$, que a fração de estrelas anãs de tipo A é maior que a de estrelas gigantes. Entretanto, a contaminação de estrelas de sequência principal mais frias também se eleva 
rapidamente nesse pequeno intervalo, razão pela qual o limite não pode se estender muito além de $(g-r)=0$. O limite inferior, $(g-r)=-0.25$, tem a intenção de incluir estrelas mais quentes, com temperaturas maiores 9500K. Os detalhes da separação de estrelas do tipo A entre anãs e gigantes são descritos no capítulo a seguir.

\subsection{Inspeção e qualidade dos espectros}

Os espectros extraídos da região cor-cor adotada na seção anterior foram inicialmente pré-inspecionados pelo Professor Timothy Beers e, posteriormente, revisitados para este trabalho, para que somente espectros estelares fossem considerados. Dos 21692 espectros inspecionados, 20526 são estelares.

Durante as observações, o mapeamento do SDSS não deixa lacunas entre os campos observados no céu. O imageamento perfaz uma superposição nas bordas dos campos observados, o que resulta em objetos idênticos classificados com nomes distintos em placas diferentes. Para evitar a utilização de estrelas iguais, espectros que possuíam coordenadas celestes idênticas foram excluídos, fornecendo assim 19771 espectros estelares únicos. Alguns exemplos de espectros selecionados com diferentes razões sinal/ruído médias $(\langle\mathrm{S} / \mathrm{R}\rangle)$ são vistos na Figura 2.4. A relação $\langle\mathrm{S} / \mathrm{R}\rangle$ adotada considera a média por pixel entre 4000-8000 ̊.

A amostra contém espectros com magnitudes aparentes no intervalo $12<g<20$. Os erros médios associados à fotometria são vistos na Figura 2.5. Pode-se notar que eles aumentam rapidamente para $g>18$. Alguns espectros não apresentam erros associados a sua fotometria. Nesses casos, o erro adotado foi equivalente a média do erro de estrelas com magnitudes equivalentes.

\subsection{Parâmetros atmosféricos - SSPP}

Os espectros selecionados foram submetidos às rotinas do SSPP para que seus parâmetros estelares fossem determinados. Os resultados foram cedidos pelos pesquisadores Young Sun Lee e Timothy Beers (comunicação particular). Como produto dos cálculos automatizados do SSPP, obteve-se para todos os espectros: temperatura efetiva $\left(T_{e f}\right)$, logarítmo da gravidade superficial $(\log (\mathrm{g}))$, metalicidade $([\mathrm{Fe} / \mathrm{H}])$ e a velocidade radial heliocêntrica $\left(V_{R_{\odot}}\right)$ 

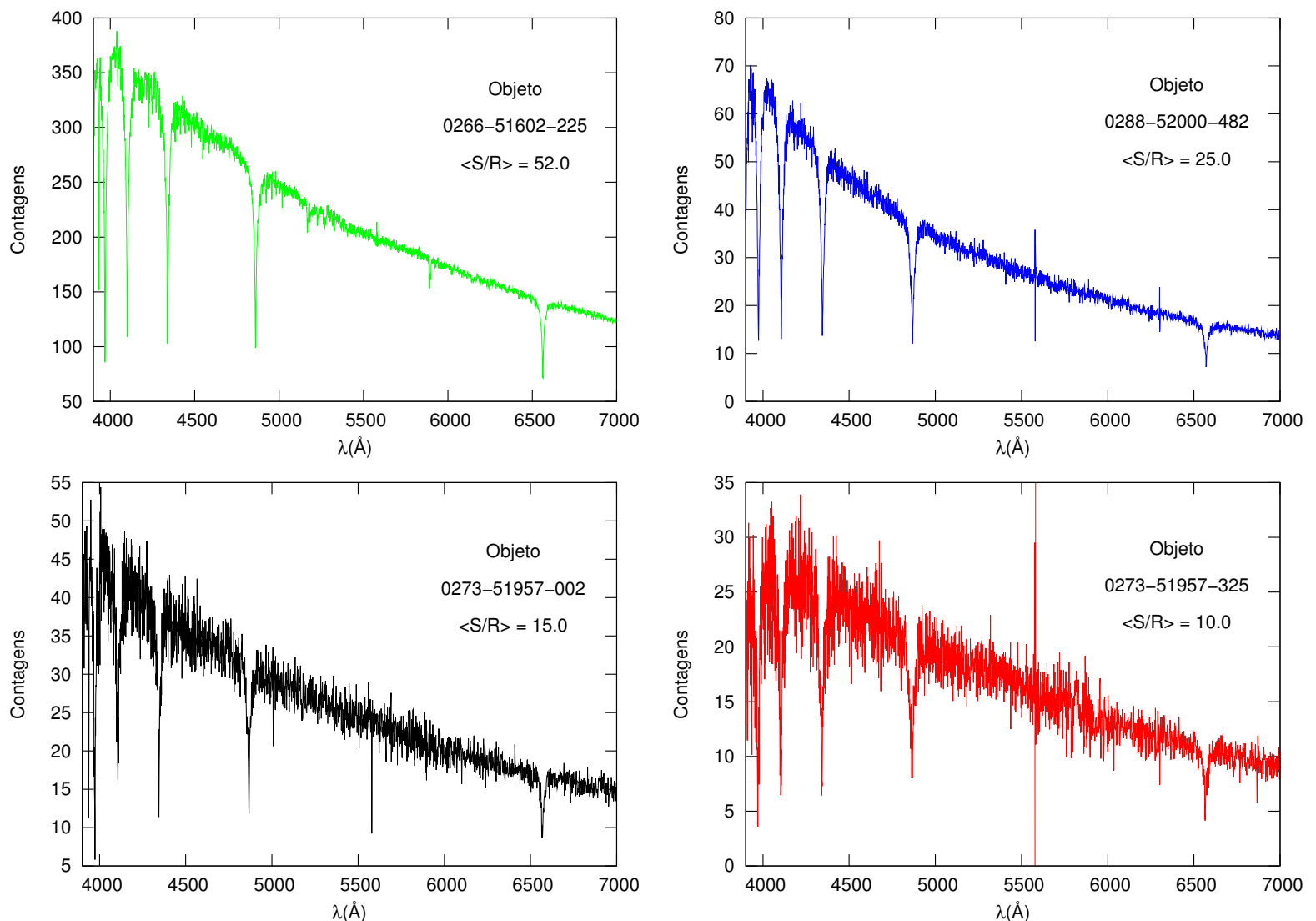

Figura 2.4: Quatro exemplos de espectros selecionados via inspeção visual. Com diferentes $\langle\mathrm{S} / \mathrm{R}\rangle$.

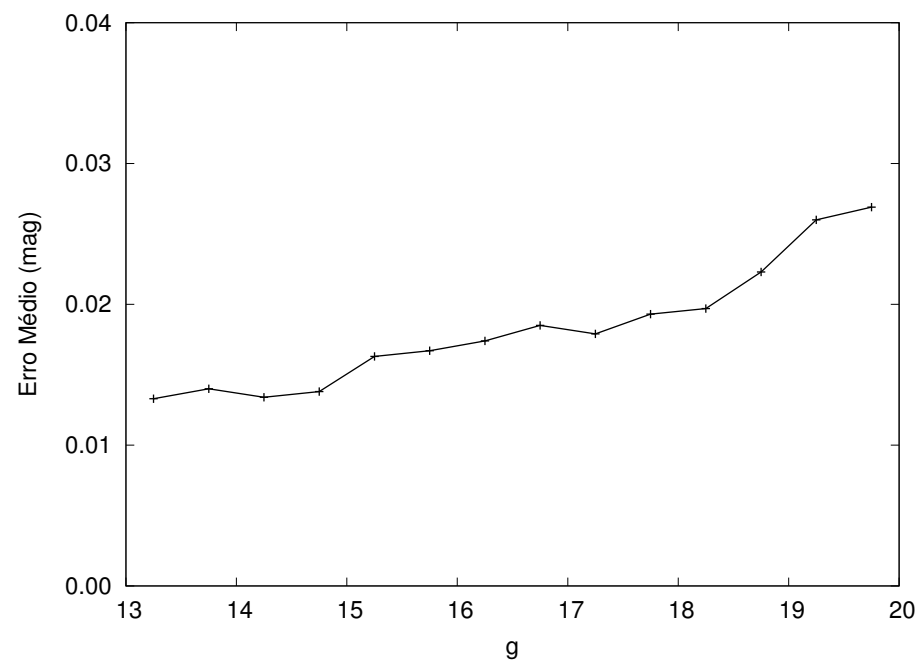

Figura 2.5: Média dos erros associados a fotometria na banda $g$. Os espectros selecionados encontram-se no intervalo $12<g<20$. 
foram estimados para todos os espectros. A Figura 2.6 apresenta a distribuição dos 19771 espectros em coordenadas galácticas.

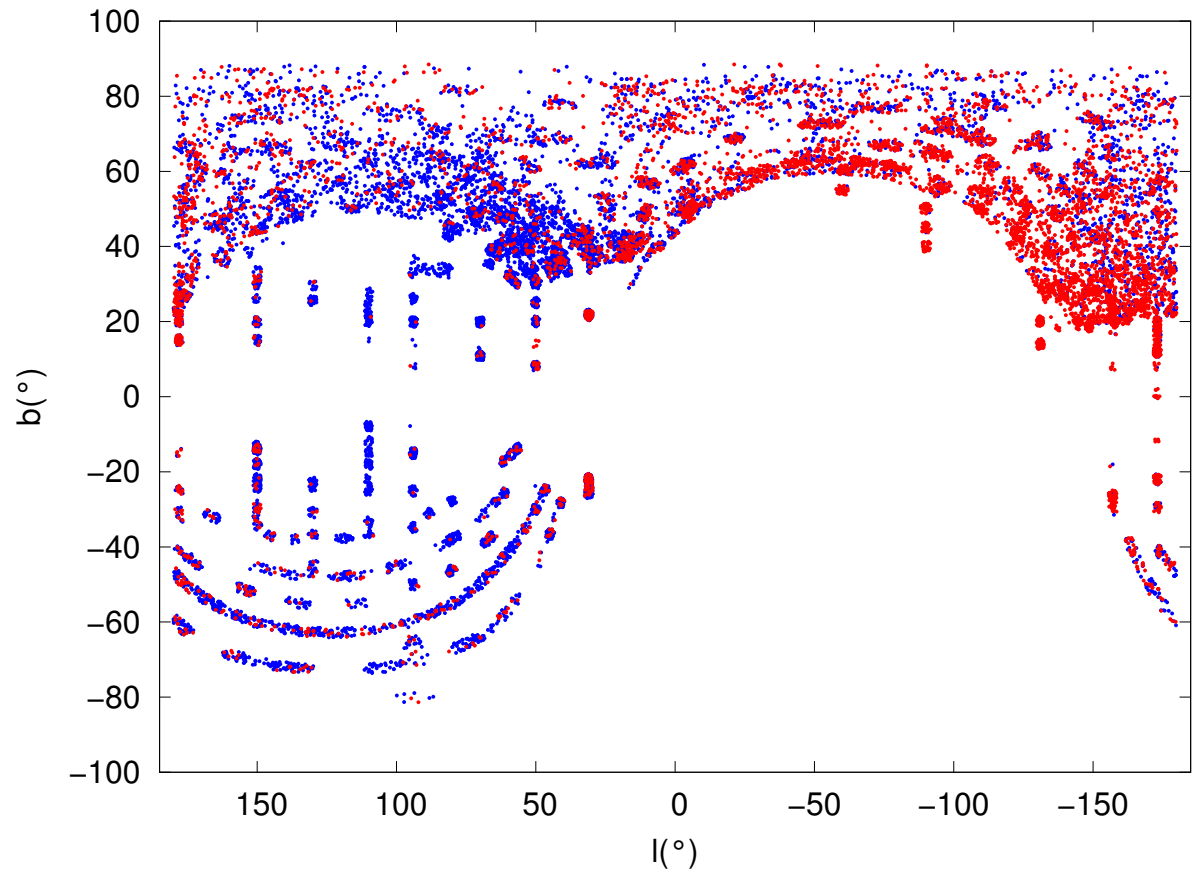

Figura 2.6: Distribuição das estrelas selecionadas do DR8 em coordenadas celestes galácticas.

Os pontos estão coloridos de acordo com o sinal da velocidade radial heliocêntrica $\left(V_{R_{\odot}}\right.$ em $\mathrm{km} / \mathrm{s}): V_{R_{\odot}}<0$ em azul e $V_{R_{\odot}}>0$ em vermelho.

Através da metalicidade estimada pelo SSPP, as estrelas de tipo espectral A foram restritas à $[\mathrm{Fe} / \mathrm{H}]<-0.4$, pois busca-se selecionar somente as estrelas pobres em metais. Inicialmente, não se sabe se os objetos selecionados são sistemas simples ou não. Entretanto, dada a alta fração de sistemas binários encontrada para as estrelas BS, o limite de metalicidade adotado utiliza como referência o enriquecimento metálico da estrela Sirius A, uma estrela de sequência principal de tipo espectral A muito próxima ( $~ 8.5$ anos-luz) e que possui uma companheira (Sirius B) anã branca. Liebert et al. (2005) estimam que Sirius B enriqueceu a metalicidade da atmosfera de Sirius A em 0.5dex, de maneira que sua metalicade, antes solar, passou a ser $[\mathrm{Fe} / \mathrm{H}]=+0.5$. O enriquecimento de 0.5 dex na metalicidade está subentendido no limite adotado para seleção em metalicidades dentro do DR8 $([\mathrm{Fe} / \mathrm{H}]<-0.4)$. No caso extremo, onde todos os objetos selecionados sejam hipoteticamente remanescentes de sistemas binários, ele apareceriam ligeiramente enriquecidos, e não como pobres em metais $([\mathrm{Fe} / \mathrm{H}] \sim-1.0)$. A Figura 2.7 mostra a distribuição 
de metalicidades da amostra calculada pelo SSPP e o local do corte para $[\mathrm{Fe} / \mathrm{H}]=-0.4$. Tal procedimento reduziu a amostra para 18560 estrelas.

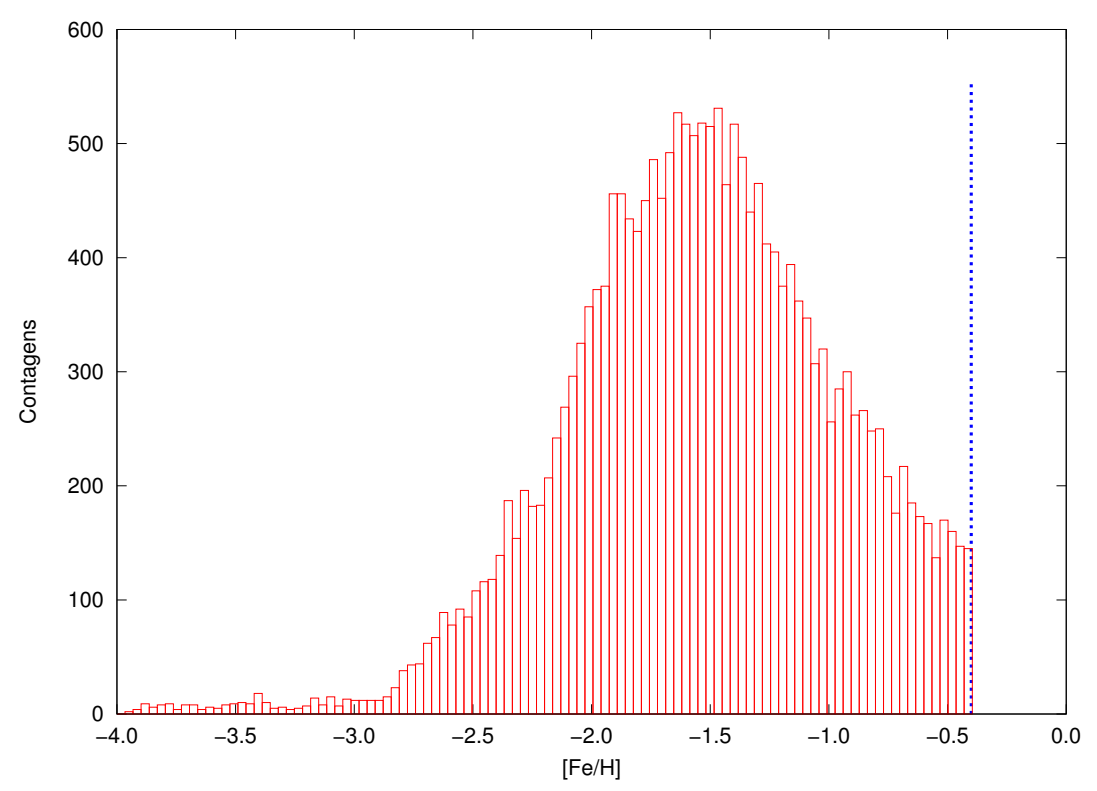

Figura 2.7: Distribuição de metalicidade da amostra com parâmetros calculados pelo SSPP. $\mathrm{O}$ corte para $[\mathrm{Fe} / \mathrm{H}]=-0.4$ é identificado pela linha azul tracejada.

O SSPP foi desenvolvido para ser aplicado a grandes bases de dados e automatizar o processo de busca e análise de estrelas pobres em metais. Entretanto, sua validade para as estrelas acima de 7500K nunca foi verificada, dada a escassez de objetos em alta resolução nessa faixa de temperatura necessários para as calibrações. No capítulo seguinte, são feitas diversas comparações qualitativas dos parâmetros calculados pelo SSPP com métodos tradicionais de separação entre estrelas anãs e gigantes, com a finalidade de avaliar sua aplicabilidade.

\subsection{As linhas da série de Balmer do Hidrogênio}

As linhas da série de Balmer do Hidrogênio são as principais ferramentas para separar estrelas (tipo A) anãs de gigantes. É justamente na faixa de temperaturas onde se encontram as estrelas de tipo espectral A que as linhas da série de Balmer são mais evidentes. A temperatura da estrela modifica a profundidade da linha de absorção do Hidrogênio, enquanto que o efeito de diferentes gravidades superficiais alteram a largura de suas asas (Mihalas, 1982). Tais fatos modificam visivelmente a aparência do espectro, conforme pode 
ser visto na Figura 2.8: quanto maior a gravidade superficial da estrela, mais larga será a asa da linha.

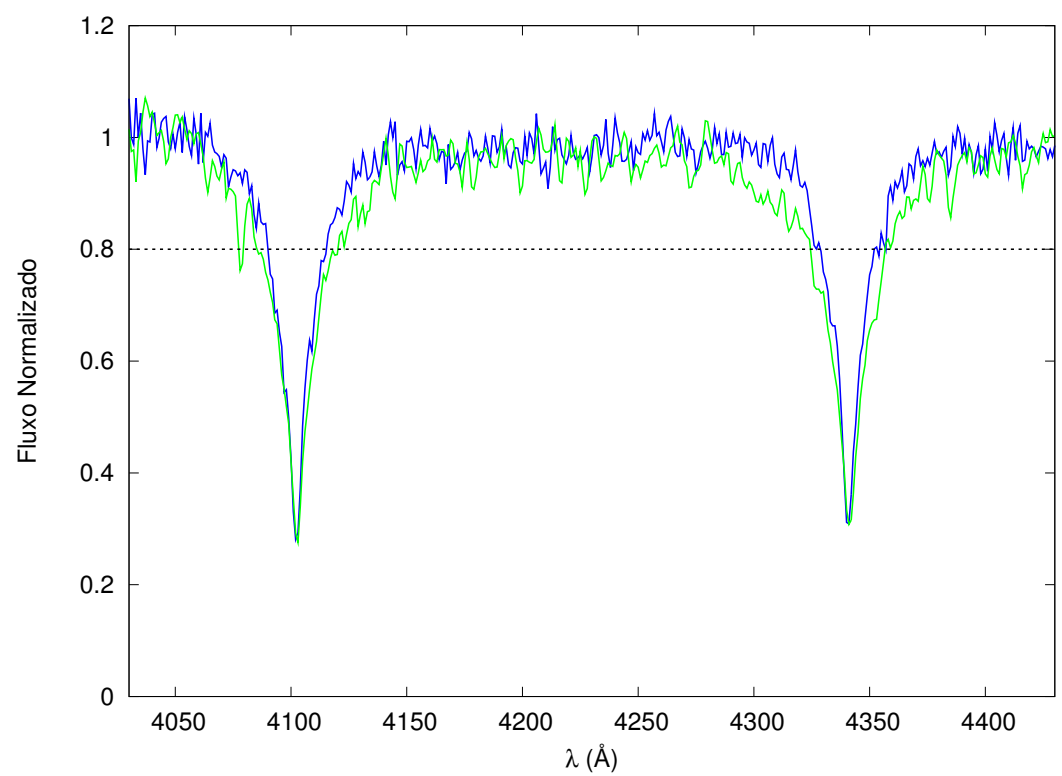

Figura 2.8: Espectro normalizado de uma estrela gigante (em azul) e de uma estrela anã (em verde) para uma mesma temperatura efetiva $(\sim 8100 \mathrm{~K})$ na região $\mathrm{H}_{\gamma}-\mathrm{H}_{\delta}$. A linha tracejada no nível $20 \%$ abaixo do contínuo mostra a diferença na largura das asas, quanto maior a gravidade superficial, maior será a largura da asa.

No capítulo a seguir, as restrições na seleção de estrelas anãs dentre as gigantes são vistas com mais detalhes. 
Capítulo 3

\section{Seleção de Estrelas BS}

Nesse capítulo são apresentados os métodos tradicionais de separação entre estrelas de tipo A e gigantes, bem como suas limitações. Nesses casos, são definidas novas restrições para selecionar estrelas BS através dos parâmetros atmosféricos estimados pelo SSPP. Os parâmetros resultantes dos ajustes neste capítulo são encontrados no Apêndice A. As estrelas que resistiram às classificações dos métodos adotados, apresentando maior confiabilidade, são avaliadas no Capítulo 4.

\subsection{Método $f_{m}$ vs $D_{0.2}$}

As primeiras soluções para separar as estrelas de tipo espectral A anãs de gigantes, fotometricamente indistinguíveis, foram propostas há décadas. O método $\mathrm{f}_{m}$ vs $\mathrm{D}_{0.2}$ (Pier, 1983; Sommer-Larsen e Christensen, 1986; Arnold e Gilmore, 1992; Flynn et al., 1994; Kinman et al., 1994; Wilhelm et al., 1999) ressalta a relação entre a temperatura da estrela e sua gravidade superficial através do perfil da linha $\mathrm{H}_{\delta}$ da série de Balmer. $\mathrm{D}_{0.2}$ é a largura medida $20 \%$ abaixo do contínuo e $\mathrm{f}_{m}$ é o valor do fluxo medido no centro da linha dividido pelo contínuo (Beers et al., 1992; Sirko et al., 2004). Assim, para estrelas de mesma temperatura, quanto maior a gravidade superficial da estrela, maior será o parâmetro $\mathrm{D}_{0.2}$.

Dentre os objetos selecionados até este momento, são encontrados espectros com diversas razões S/R (ver: Figura 3.1). Entretanto, para utilizar o método acima, os objetos necessitam ter razão $\langle\mathrm{S} / \mathrm{R}\rangle$ maior que 9 , pois o nível $20 \%$ deve estar livre de ruído proveniente do contínuo. Com isso, de 18560 objetos selecionados até então, 15757 satisfazem essa restrição.

Os parâmetros utilizados pelo método são obtidos através do ajuste de uma função que 


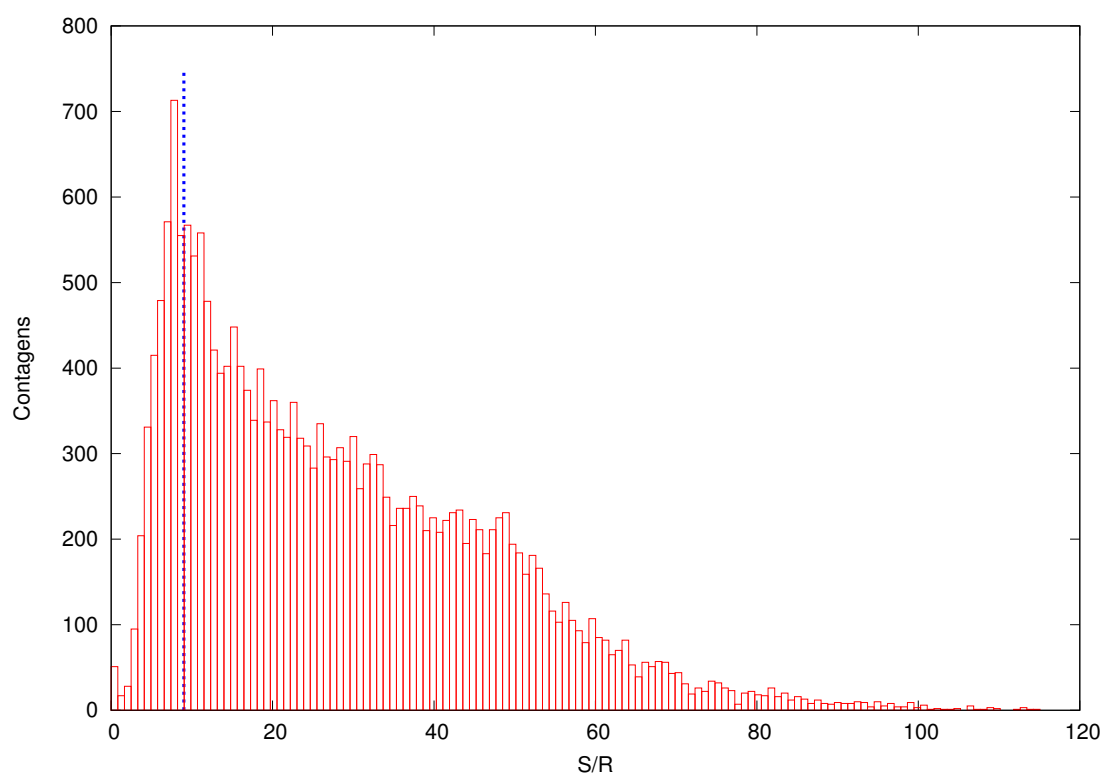

Figura 3.1: Distribuição da razão $\langle\mathrm{S} / \mathrm{R}\rangle$ dos espectros utilizados. A linha tracejada azul mostra o limite em $\langle\mathrm{S} / \mathrm{R}\rangle$ acima do qual o método $\mathrm{D}_{0.2}$ é aplicável.

descreve a forma da linha, utilizando pontos do espectro observado em um intervalo de 100 angstrons centrado na linha. A função que o método adota para esse ajuste é o chamado perfil de Sérsic (Sersic, 1968), pois se adequa de maneira versátil aos espectros em baixa resolução (Clewley et al., 2002):

$$
S(x)=n-a e^{-\left(\frac{\left|x-x_{0}\right|}{b}\right)^{c}},
$$

onde $n, a, b$ e $c$ são parâmetros livres, sendo $n$ o nível do contínuo ajustado, $x_{0}$ o comprimento de onda no centro da linha e $a$ define a profundidade. Os parâmetros $b$ (parâmetro de largura) e $c$ (parâmetro de forma) serão examinados na seção a seguir. Eles são utilizados de maneira complementar ao método $\mathrm{f}_{m}$ vs $\mathrm{D}_{0.2}$, também com o objetivo de separar as estrelas anãs de estrelas gigantes (Clewley et al., 2002).

Como o perfil de Sérsic possui um patamar (nível do contínuo) constante $n$, é necessário normalizar os espectros para que os parâmetros obtidos através dele não tenham erros associados ao desnivelamento do contínuo (Figura 3.2). A normalização faz com que o nível do contínuo seja o mesmo em ambos os lados das linhas de Hidrogênio:

Embora não exista relação fundamental entre o parâmetro $a$ e os demais parâmetros estimados no ajuste de Sérsic, verifica-se (Figura 3.3) que ele possui uma distribuição 


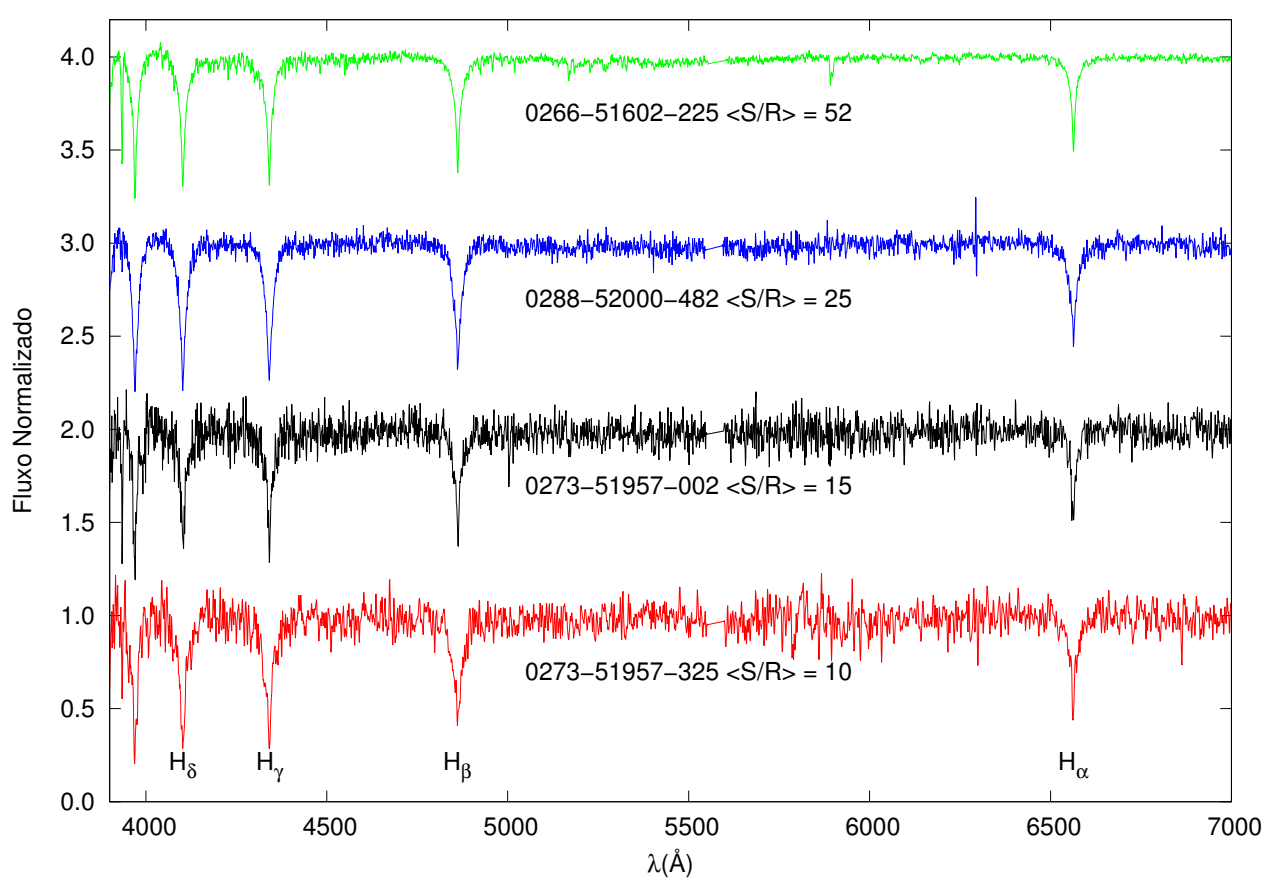

Figura 3.2: Espectros da Figura 2.4 normalizados e com intensidade de fluxo deslocada.

Com o nível do contínuo constante, os ajustes se Sérsic tem erros menores.

normal em torno de um valor médio para cada linha da série de Balmer, sendo: $\left\langle\mathrm{a}_{\alpha}\right\rangle=$ $0.574,\left\langle\mathrm{a}_{\beta}\right\rangle=0.709,\left\langle\mathrm{a}_{\gamma}\right\rangle=0.694 \mathrm{e}\left\langle\mathrm{a}_{\delta}\right\rangle=0.769$.

Dessa forma, pode-se refinar os parâmetros do ajuste de Sérsic, re-ajustando a função com o valor de $\langle a\rangle$ fixo para cada linha (ver: Figura 3.4), enquanto $\mathrm{f}_{m}, \mathrm{D}_{0.2}, b$ e $c$ são aprimorados para as linhas $\mathrm{H}_{\alpha}, \mathrm{H}_{\beta}, \mathrm{H}_{\gamma}$ e $\mathrm{H}_{\delta}$. Sendo que, $\mathrm{D}_{0.2}$ é obtido a partir da função inversa de $S(x)$ :

$$
D_{0.2}=2 b\left(-\log \left(\frac{0.2 n}{n-\langle a\rangle}\right)\right)^{\frac{1}{c}} .
$$

A Figura 3.5 mostra a relação dos parâmetros encontrados nos ajustes para as linhas da série de Balmer avaliadas. Nota-se que a linha $\mathrm{H}_{\delta}$ revela os maiores contrastes entre os dois tipos de estrelas (Sirko et al., 2004), sendo portanto a adotada no método $\mathrm{f}_{m}, \mathrm{D}_{0.2}$, como visto no painel inferior direito da Figura 3.5.

Através da linha $\mathrm{H}_{\delta}$, são vistos dois grupos de estrelas espalhados em torno de $\mathrm{f}_{m \delta}$ $\sim 0.26$ : as estrelas gigantes, que apresentam largura menor $(\sim 25 \AA)$, e as estrelas anãs $(\sim 35 \AA)$.

Alguns trabalhos têm utilizado limites diferentes para restringir as distribuições vistas 

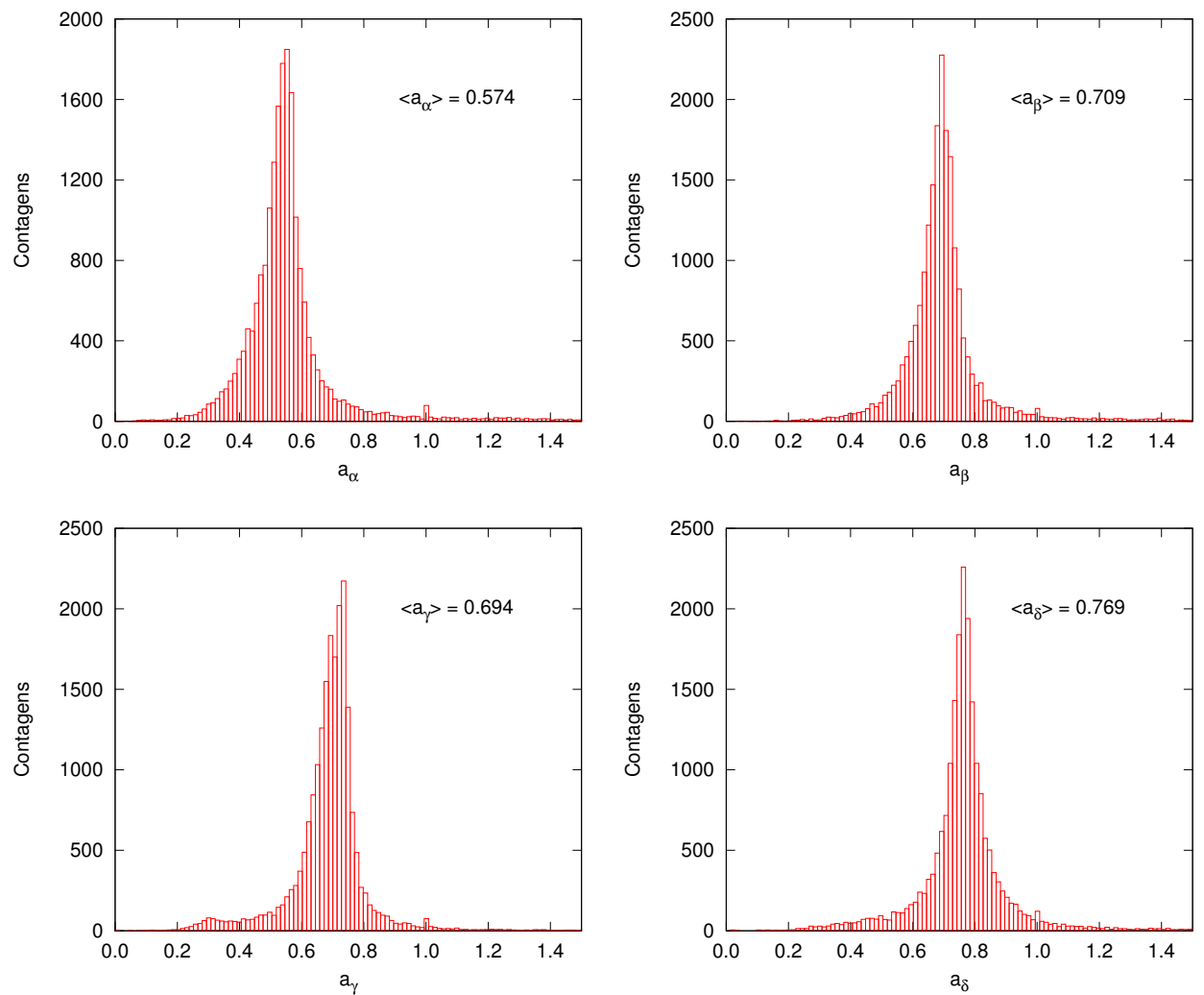

Figura 3.3: Distribuição normal dos parâmetros $a$, calculados pelo perfil de Sérsic, para as diferentes linhas do $\mathrm{H}$.

na Figura 3.5 (Sirko et al., 2004; Xue et al., 2008), já que nenhum deles visa selecionar as estrelas anãs, mas sim excluí-las. Além disso, Sirko et al. (2004) mostraram que o método $\mathrm{f}_{m \delta}$ vs $\mathrm{D}_{0.2 \delta}$, mesmo aplicável à estrelas com $g>18$, não é capaz de revelar nitidamente a separação entre anãs e gigantes, devido à baixa razão $\mathrm{S} / \mathrm{R}$ desses objetos (ver painéis esquerdos da Figura 3.19. Assim, somente 10094 satisfazem a restrição em magnitude.

Na Figura 3.6 apresenta-se os limites físicos de separação estimados tradicionalmente (Clewley et al., 2002; Sirko et al., 2004; Xue et al., 2008) com pequenas modificações, pois aqui o objetivo é separar as estrelas anãs com a máxima eficiência e confiança possíveis. Estrelas BHB raramente habitam a região de $\mathrm{f}_{m \delta}>0.35$, pois nela encontram-se estrelas mais frias. As linhas da série de Balmer diminuem de intensidade (tanto a largura como a profundidade) de tal forma que a região $\mathrm{D}_{0.2 \delta}<20 \AA$ e $\mathrm{f}_{m \delta}>0.3$ é dominada por estrelas mais frias, de tipos F, G e até K (Clewley et al., 2002; Sirko et al., 2004). 

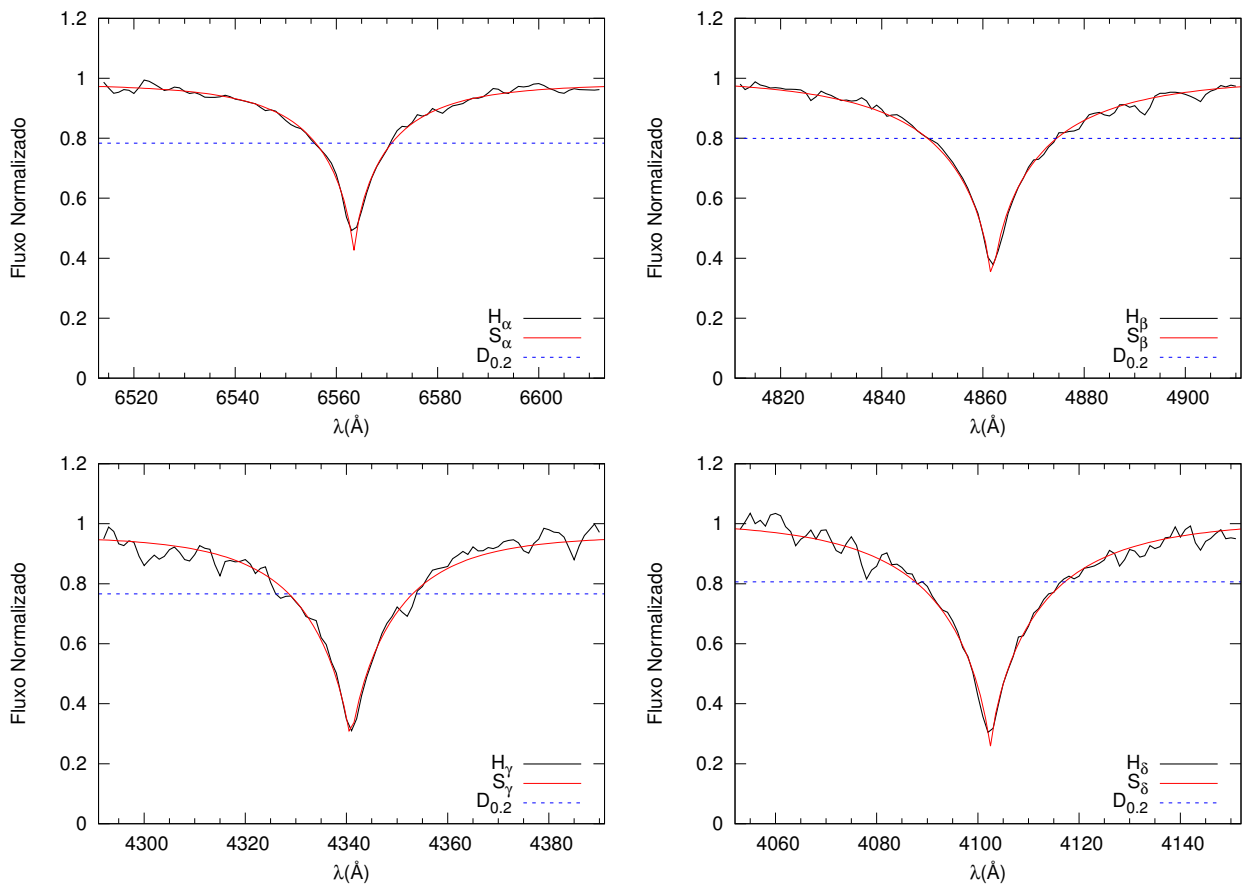

Figura 3.4: Ajuste de Sérsic na região da série de Balmer. A linha preta representa o espectro observado, a linha vermelha mostra o ajuste do perfil de Sérsic (para $\langle a\rangle$ fixo) e a linha azul tracejada mostra o nível $20 \%$ abaixo do contínuo.

\subsection{Método de forma e largura ( $c$ vs $b$ )}

O método de forma e largura ( $c$ vs b), descrito primeiramente por Clewley et al. (2002), separa as estrelas anãs de gigantes através dos parâmetros ajustados no perfil de Sérsic, e o faz, preferencialmente, para estrelas com magnitude $g<18$. Apesar de ser semelhante ao método $\mathrm{f}_{m \delta}$ vs $\mathrm{D}_{0.2_{\delta}}$, ele se diferencia por utilizar os parâmetros da linha $\mathrm{H}_{\gamma}$, como pode ser visto na Figura 3.7 (painel inferior esquerdo). A linha $\mathrm{H}_{\gamma}$ é escolhida para ser avaliada no método de forma e largura pois, dentre as linhas da série de Balmer analisadas na região $\mathrm{H}_{\alpha}-\mathrm{H}_{\delta}$, é a que possui a melhor distinção entre estrelas anãs e gigantes no método de forma e largura (Figura 3.7).

Nota-se que a linha $\mathrm{H}_{\alpha}$ é incapaz de separar as estrelas anãs de gigantes por ser pouco intensa. Além disso, como método de forma e largura é complementar ao método $\mathrm{f}_{m \delta}$ vs $\mathrm{D}_{0.2 \delta}$, a linha $\mathrm{H}_{\delta}$ não pode ser considerada isoladamente (Clewley et al., 2002; Sirko et al., 2004). A linha $\mathrm{H}_{\epsilon}$ não é utilizada pois se justapõe à linha $\mathrm{H}$ do Ca II. As outras linhas da série de Balmer estão muito próximas entre si para que o ajuste de Sérsic consiga 


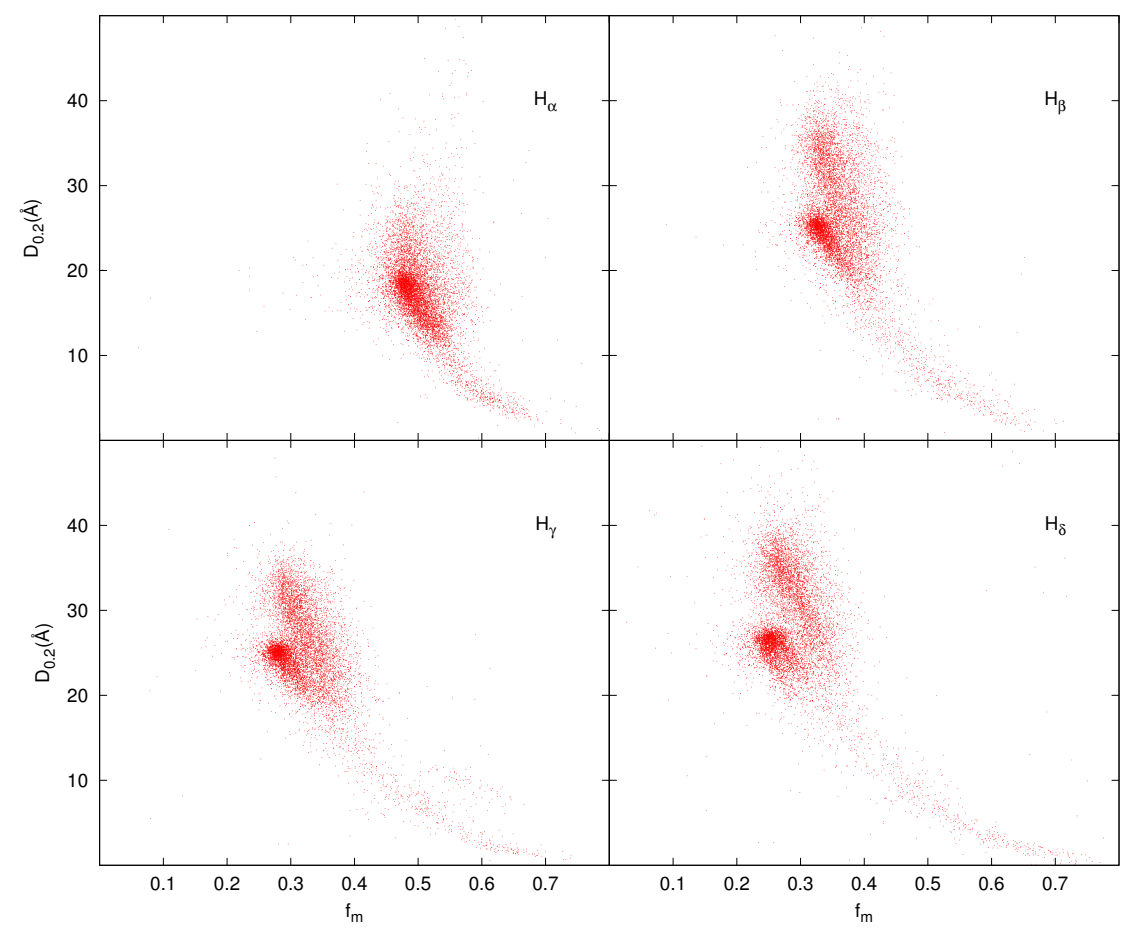

Figura 3.5: Gráfico dos parâmetros $\mathrm{f}_{m}$ vs $\mathrm{D}_{0.2}$ para as linhas $\mathrm{H}_{\alpha}, \mathrm{H}_{\beta}, \mathrm{H}_{\gamma}$ e $\mathrm{H}_{\delta}$ da série de Balmer. Dentre as linhas avaliadas, aquela que apresenta a maior distinção entre dois grupos de estrelas é a $\mathrm{H}_{\delta}$.

descrevê-las isoladamente.

Com a finalidade de realçar a separação entre as estrelas gigantes e anãs no método $c_{\gamma}$ vs $b_{\gamma}$, adota-se a média dos parâmetros de forma e largura para as linhas $\mathrm{H}_{\beta}, \mathrm{H}_{\gamma}$ e $\mathrm{H}_{\delta}$, conforme proposta inicialmente feita por Deason et al. (2011) (ver Figura 3.8).

Os métodos $\mathrm{f}_{m \delta}$ vs $\mathrm{D}_{0.2 \delta}$ e $c_{\beta \gamma \delta}$ vs $b_{\beta \gamma \delta}$ são os métodos clássicos usados conjuntamente para separar as estrelas (tipo A) anãs (BS) de estrelas gigantes (BHB). A combinação deles é apresentada na Figura 3.8, onde os parâmetros $c_{\beta \gamma \delta}$ e $b_{\beta \gamma \delta}$ são coloridos em função das regiões descritas pelo método $\mathrm{f}_{m \delta}$ vs $\mathrm{D}_{0.2 \delta}$ (Figura 3.6).

A utilização da média dos parâmetros de forma e largura para as linhas $\mathrm{H}_{\beta}, \mathrm{H}_{\gamma}$ e $\mathrm{H}_{\delta}$ não torna mais o método $c$ vs $b$ independente do método $\mathrm{f}_{m \delta}$ vs $\mathrm{D}_{0.2 \delta}$, pois a linha $\mathrm{H}_{\delta}$ é utilizada por ambos os métodos. Logo, é necessário buscar uma alternativa para avaliar os erros quando se combina os métodos tradicionais. A seção a seguir define um critério direto para avaliar a separação entre as estrelas anãs e gigantes através do SSPP. 


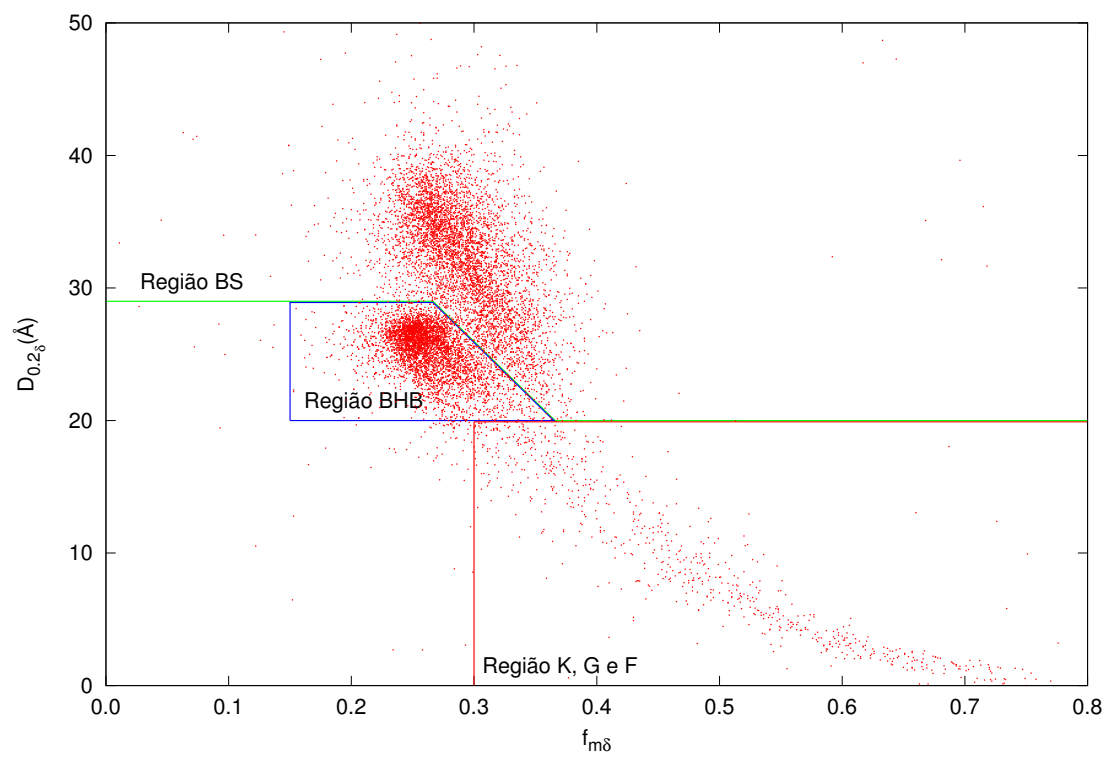

Figura 3.6: Gráfico dos parâmetros $\mathrm{f}_{m \delta}$ vs $\mathrm{D}_{0.2 \delta}$ com magnitude $g<18$. Nota-se que a dispersão diminui em virtude da limitação de objetos menos brilhantes, cuja razão $\langle\mathrm{S} / \mathrm{R}\rangle$ é menor. O retângulo em vermelho é povoado por estrelas de tipo F, G e K. A região comum às estrelas gigantes BHB é vista na área azul e a região superior, em verde, contém as candidatas a estrelas BS.

\subsection{Método do SSPP}

Com o advento do Segue Stellar Parameter Pipeline (SSPP) do SDSS, é possível comparar diretamente $\mathrm{T}_{\text {ef }} \operatorname{com} \mathrm{o} \log (\mathrm{g})$ dos objetos (Figura 3.9) de forma independente dos métodos clássicos descritos anteriormente. Tal comparação foi proposta incialmente por Wilhelm et al. (1999), utilizando métodos espectrofotométricos que, posteriormente foram incorporados também ao SSPP como parte de suas rotinas. Entretanto, para estimar os parâmetros atmosféricos estelares para $\mathrm{T}_{e f}>7500 \mathrm{~K}$, o SSPP faz correlações e iterações com outros métodos resultando em erros menores (ver: Lee et al., 2008; Allende Prieto et al., 2008, para mais detalhes).

A gravidade superficial (g) é medida, normalmente, no Sistema de Unidades Internacional (SI) em metros por segundo ao quadrado $\left(\mathrm{m} / \mathrm{s}^{2}\right)$. Entretanto, em astronomia, adota-se o uso do $\log (\mathrm{g})$, e nesse caso, a gravidade superficial é expressa no sistema de unidades CGS. As estrelas de tipo A na sequência principal possuem valores de $\log (\mathrm{g})$ parecidos com o Solar $\left(\log (\mathrm{g})_{\odot}=4.44\right)$, ou seja, típicos de estrelas anãs, entre 3.8 e 4.5 dex 

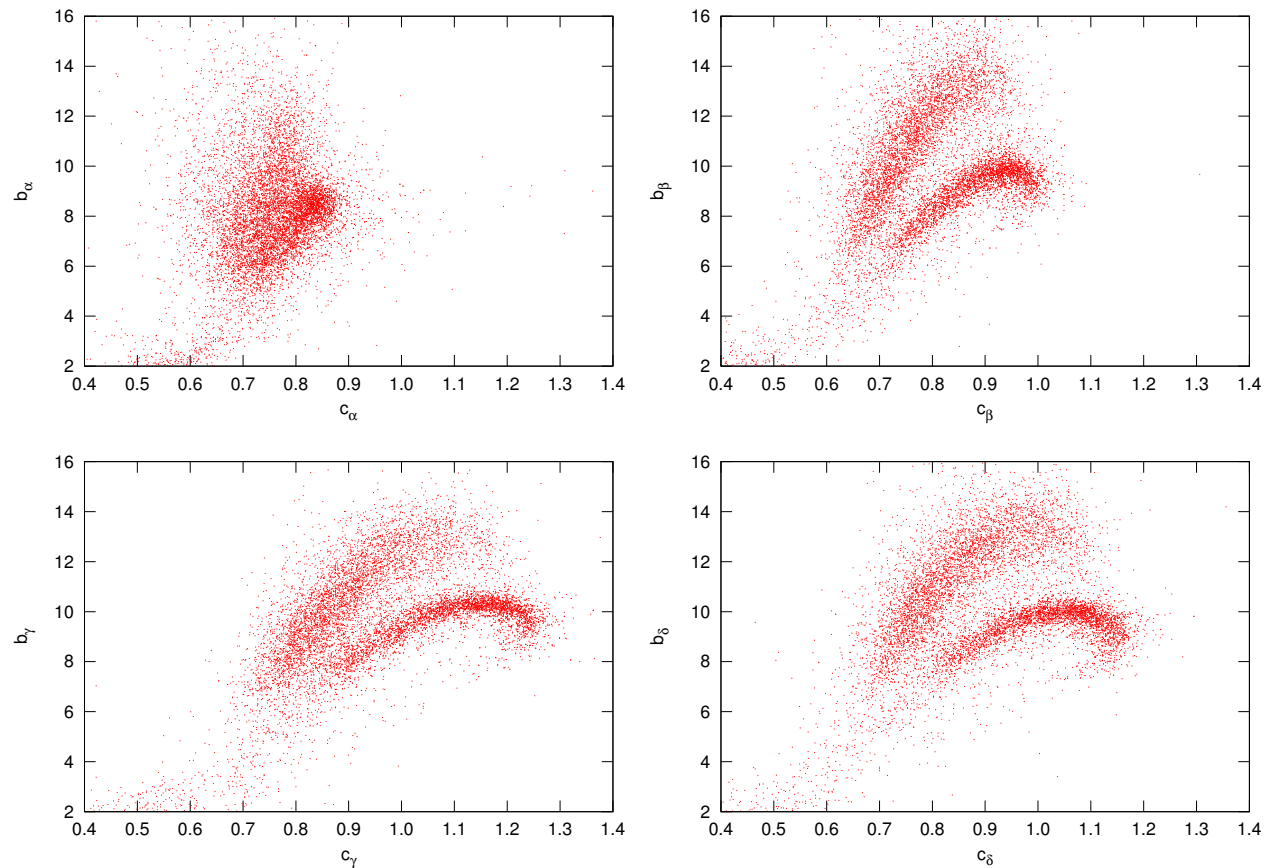

Figura 3.7: Parâmetros de forma e largura ( $c$ vs b) para as linhas $\mathrm{H}_{\alpha}, \mathrm{H}_{\beta}, \mathrm{H}_{\gamma}$ e $\mathrm{H}_{\delta}$. A linha $\mathrm{H}_{\alpha}$ é incapaz de mostra qualquer distinção entre estrelas anãs e gigantes.

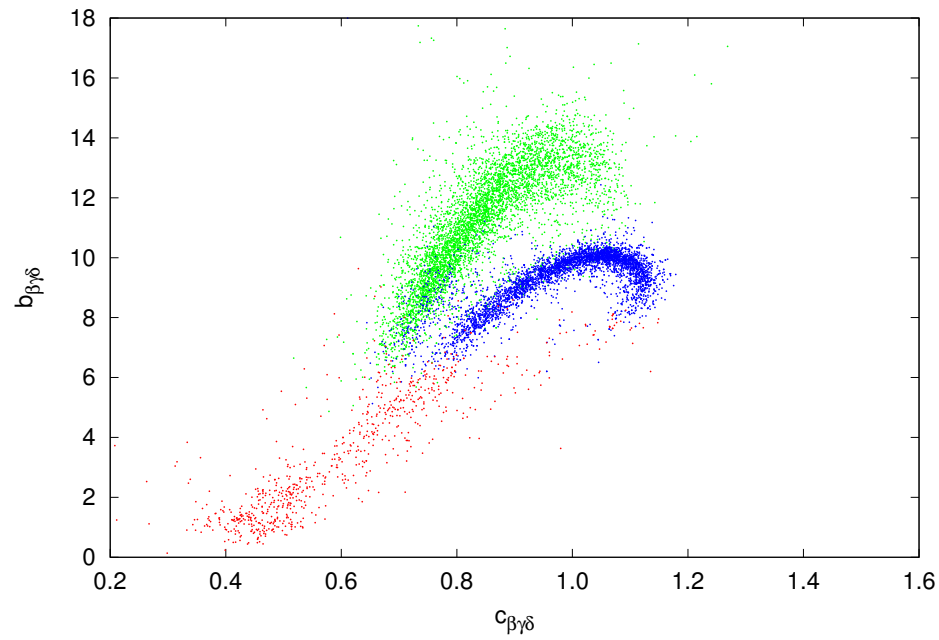

Figura 3.8: Média dos parâmetros forma e largura ( $c$ vs b) para as linhas $\mathrm{H}_{\beta}, \mathrm{H}_{\gamma}$ e $\mathrm{H}_{\delta}$. Os pontos são coloridos de acordo com as regiões ressaltadas na Figura 3.6. Pontos verdes representam as estrelas classificadas como azuis tardias (BS), pontos azuis as estrelas gigantes (BHB) e os pontos vermelhos as estrelas de tipo F, G e K.

(Adelman, 2004). Já para as estrelas gigantes e subgigantes, o $\log (\mathrm{g})$ típico varia entre 3.0 e 3.8 dex. 


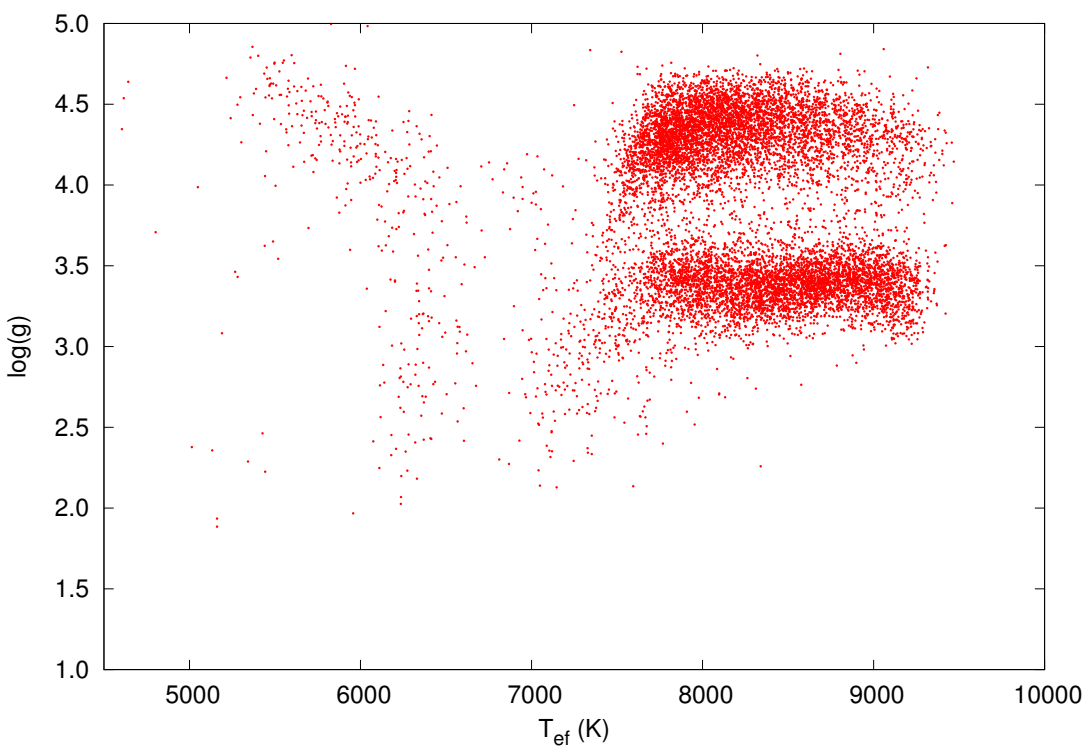

Figura 3.9: Relação entre os parâmetros atmosféricos estimados pelo SSPP do SDSS. Podese notar dois grupos de estrelas concentrados na região $\mathrm{T} \geq 7500 \mathrm{~K}$ : um com $\log (\mathrm{g})$ típico de gigantes $\sim 3.4$ dex e outro $\operatorname{com} \log (\mathrm{g})$ típico de estrelas na sequência principal $\sim 4.4$ dex.

A Figura 3.10 mostra a distribuição de $\log (\mathrm{g})$ do dados utilizados na Figura 3.9. A distribuição revela dois picos que foram ajustados por funções gaussianas. O primeiro deles, para $\log (\mathrm{g})=3.38$, é típico de estrelas gigantes, enquanto o outro, centrado em $\log (\mathrm{g})=$ 4.33, possui valores de gravidade superficial de estrelas anãs. As distribuições de $\log (\mathrm{g})$ representadas ao longo da análise, são ajustadas por funções gaussianas. No Apêndice A são encontrados os parâmetros dos ajustes e seus respectivos erros. O Apêndice B resume o critério de escolha da largura dos bins utilizado neste trabalho.

Para selecionar estrelas anãs (BS) a partir do $\log (\mathrm{g})$ do SSPP (uma vez que as estrelas do tipo A possuem temperaturas efetivas entre $7500 \mathrm{~K}<\mathrm{T}_{e f}<10000 \mathrm{~K}$ ), considera-se que a região $3 \sigma_{B H B}$ acima de $\log (\mathrm{g})_{B H B}=3.385$ marca o limite onde $99,7 \%$ das estrelas $\mathrm{BHB}$ estão contidas. Assim, para $\log (\mathrm{g}) \geq 3.8$, a contaminação de estrelas gigantes é menor que 0.3\%. Esses critérios estão resumidos na Figura 3.11, onde as regiões destacadas marcam os limites para cada grupo.

As Figuras 3.12 e 3.13 mostram, respectivamente, a classificação adotada pelo SSPP para os métodos $\mathrm{f}_{m \delta}$ vs $\mathrm{D}_{0.2 \delta}$ e $c_{\beta \gamma \delta}$ vs $b_{\beta \gamma \delta}$. Ambos têm concordância com as restrições definidas pelo SSPP. 


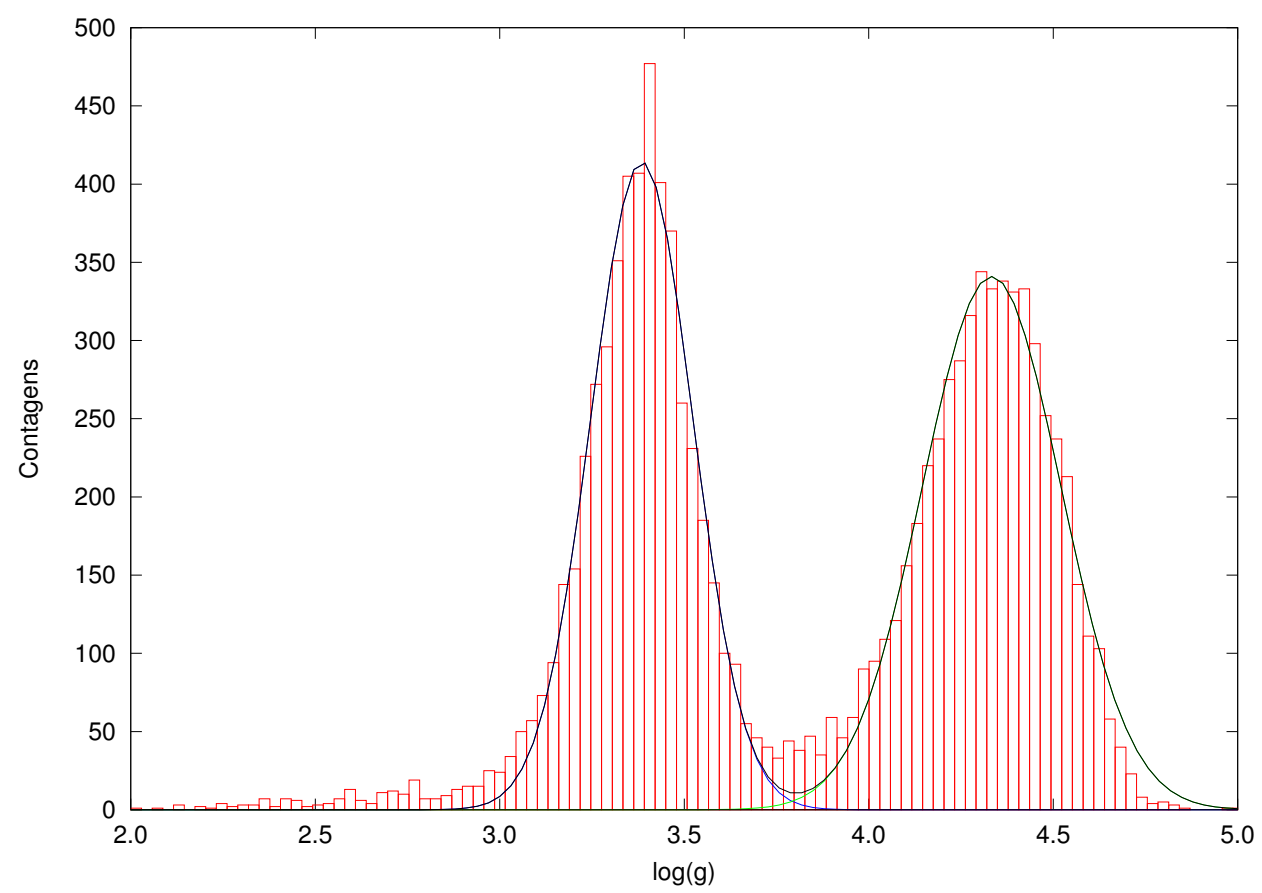

Figura 3.10: Distribuição de $\log (\mathrm{g})(g<18)$ de parâmetros fornecidos pelo SSPP. O pico centrado em $\log (\mathrm{g})=3.385(\sigma=0.138)$ representa a distribuição de estrelas gigantes e $\log (\mathrm{g})$ $=4.333(\sigma=0.188)$ as estrelas anãs.

O pipeline revela também que seus critérios são mais eficientes em separar as estrelas anãs de gigantes quando comparado com o método método de forma e largura, como pode ser visto na Figura 3.13, pois a contaminação de estrelas BHB na região das anãs (BS) é ainda mais reduzida. A distribuição das estrelas classificadas pelo SSPP no diagrama cor-cor é apresentada na Figura 3.14. Como descrito no capítulo anterior, o limite do índice $(g-r)$ foi estendido em relação aos valores tradicionais com a finalidade de incluir um número maior de estrelas anãs.

\subsection{O SSPP para estrelas quentes}

O SSPP é basicamente utilizado para extrair os principais parâmetros físicos de estrelas pobres em metais com temperaturas efetivas menores que $7500 \mathrm{~K}$ (Lee et al., 2008). Como os objetos selecionados neste estudo encontram-se acima desse limite de temperatura efetiva, foi necessário avaliar sua aplicabilidade à amostra.

Uma vez que o parâmetro $\mathrm{f}_{m \delta}$ fornece uma estimativa indireta da temperatura efetiva, 


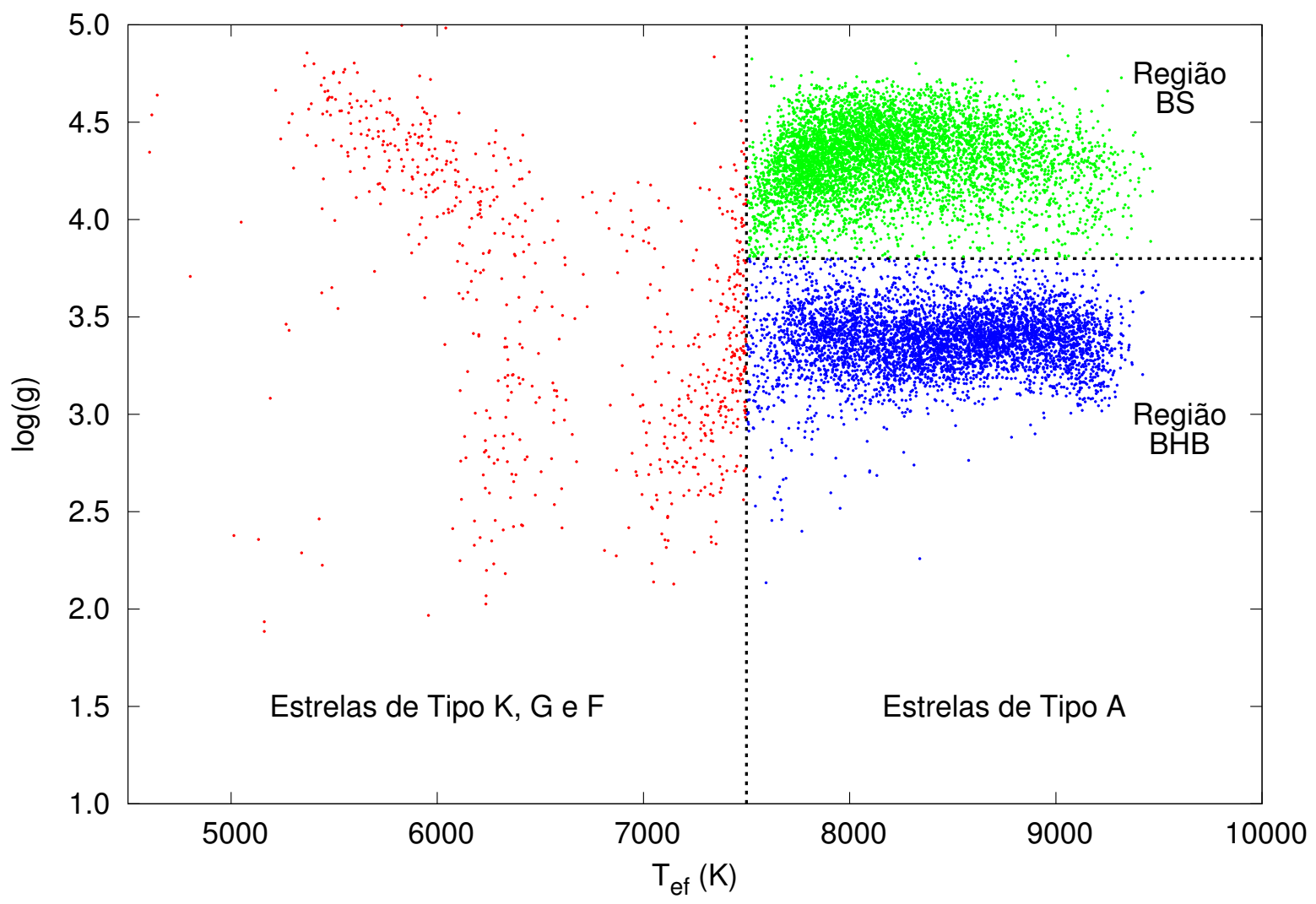

Figura 3.11: Relação entre os parâmetros $\mathrm{T}_{\text {ef }}$ e $\log (\mathrm{g})$ estimados pelo SSPP. A região do gráfico para $\log (\mathrm{g})>3.8$ e $\mathrm{T}_{e f}>7500 \mathrm{~K}$ limita a área comum de estrelas do tipo $\mathrm{A}$ anãs (BS). Os pontos verdes representam as estrelas classificadas como BS pelo método do SSPP, em azul estão representadas as estrelas gigantes (BHB) e em vermelho as estrelas mais frias que $7500 \mathrm{~K}$.

espera-se que exista uma relação entre ele e a temperatura efetiva estimada pelo SSPP. A Figura 3.15 mostra que tal associação é visível, sendo plausível desenvolver uma calibração para estimar a temperatura efetiva através do parâmetro $\mathrm{D}_{0.2 \delta}$. Com isso, considera-se válida a utilização da temperatura efetiva fornecida pelo SSPP para estrelas da amostra deste estudo, e seu erro médio avaliado para estrelas com diversas razões $\langle\mathrm{S} / \mathrm{R}\rangle$ é igual a $157 \mathrm{~K}$.

Os parâmetros $\mathrm{D}_{0.2 \delta}$ e $b_{\beta \gamma \delta}$ são indicadores indiretos da gravidade superficial da estrela. Como dito anteriormente, ao adotar a média dos parâmetros $c$ e $b$ para as linhas $\mathrm{H}_{\beta}$, $\mathrm{H}_{\gamma}$ e $\mathrm{H}_{\delta}$ da série de Balmer, o método de forma e largura não é mais independente do método $\mathrm{f}_{m \delta}$ vs $\mathrm{D}_{0.2 \delta}$. A comparação entre esses parâmetros é apresentada na Figura 3.16. 


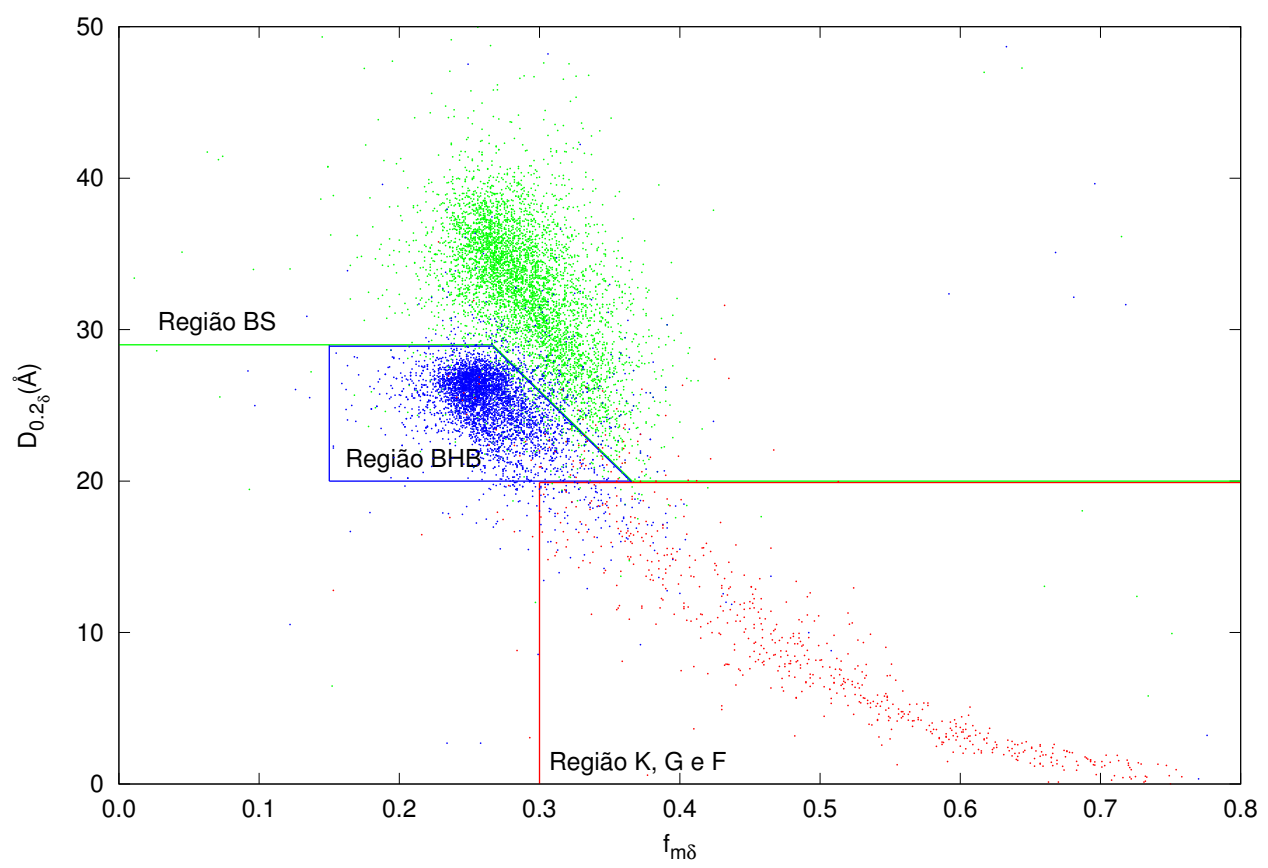

Figura 3.12: Gráfico dos parâmetros $\mathrm{f}_{m \delta}$ vs $\mathrm{D}_{0.2 \delta}$ colorido de acordo com as restrições adotadas pelo SSPP. Vemos que as dispersões encontradas para as estrelas gigantes e anãs se sobrepõe além dos limites adotados pelo método $\mathrm{f}_{m \delta}$ vs $\mathrm{D}_{0.2 \delta}$.

Embora exista, uma forte correlação entre os parâmetros, a separação entre os dois grupos de estrelas não ocorre.

Ao contrário da relação entre os métodos tradicionais, quando é feita a comparação com o SSPP, não há correlação entre os parâmetros $\mathrm{D}_{0.2 \delta}$ ou $b_{\beta \gamma \delta} \operatorname{com}$ o $\log (\mathrm{g})$. Entretanto, as diferenças entre as duas populações estelares são evidentes, como mostra a Figura 3.17. O SSPP fornece uma medida direta da gravidade da estrela e separa as estrelas de tipo A anãs de gigantes independente de outros parâmetros físicos, enquanto os métodos clássicos fazem a separação de maneira indireta e fortemente dependente da temperatura. Portanto, ao considerar as separações entre as populações estelares vistas na Figura 3.17, os métodos clássicos adotados e o método do SSPP se mostram independentes.

A gravidade superficial fornecida pelo SSPP é válida para a amostra e nota-se sua coerência quando se compara o resultado da classificação de estrelas anãs entre os dois métodos métodos classicos e o SSPP, como mostraram as Figuras 3.13 e 3.12, eles são absolutamente coincidentes. Além disso, os valores de $\log (\mathrm{g})$ estimadas pelo SSPP se enquadram perfeitamente nos intervalos teóricos vistos anteriormente (Adelman, 2004), e 


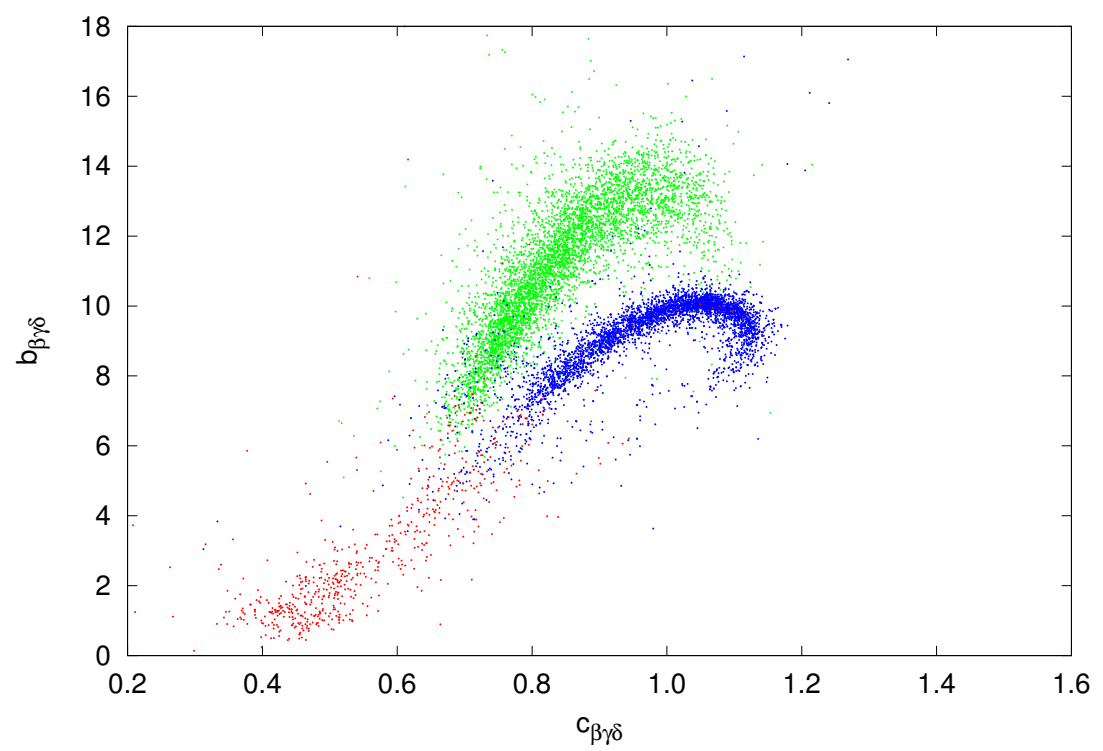

Figura 3.13: Relação entre os parâmetros $c_{\beta \gamma \delta}$ vs $b_{\beta \gamma \delta}$ coloridos de acordo com restrições adotadas pelo SSPP. O método que utiliza as restrições do SSPP se mostra mais eficiente para separar as estrelas anãs de gigantes no método de forma e largura, pois suas restrições mostram contaminações menores entre os ramos (anãs e gigantes) do que quando utilizamos as restrições do método $\mathrm{f}_{m \delta}$ vs $\mathrm{D}_{0.2 \delta}$.

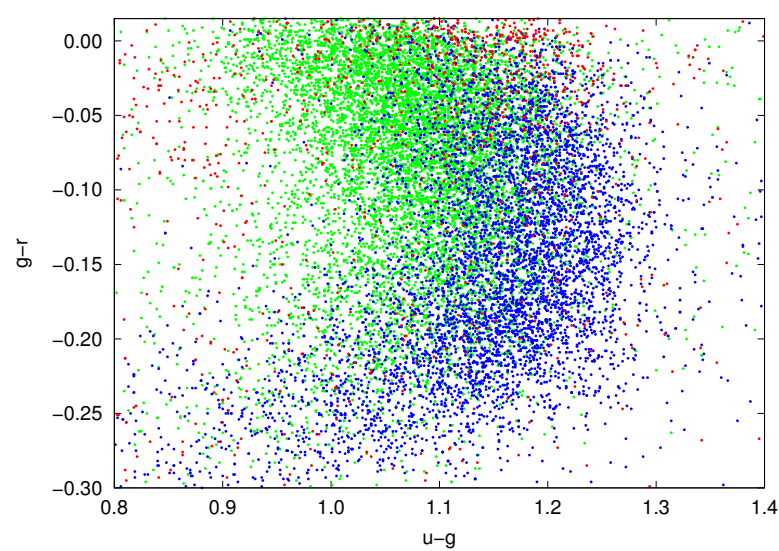

Figura 3.14: Região do diagrama cor-cor colorida de acordo com a classificação do SSPP: Os pontos em verde representam as estrelas anãs candidatas a estrelas BS, em azul estão representadas as estrelas $\mathrm{BHB}$ e em vermelho estrelas de tipo espectral F, G e K.

exemplificados na Figura 3.10.

Além dos parâmetros atmosféricos, a metalicidade fornecida pelo SSPP pode ser verificada qualitativamente utilizando-se espectros de estrelas quentes ( $~ 8600 \mathrm{~K})$, através da 


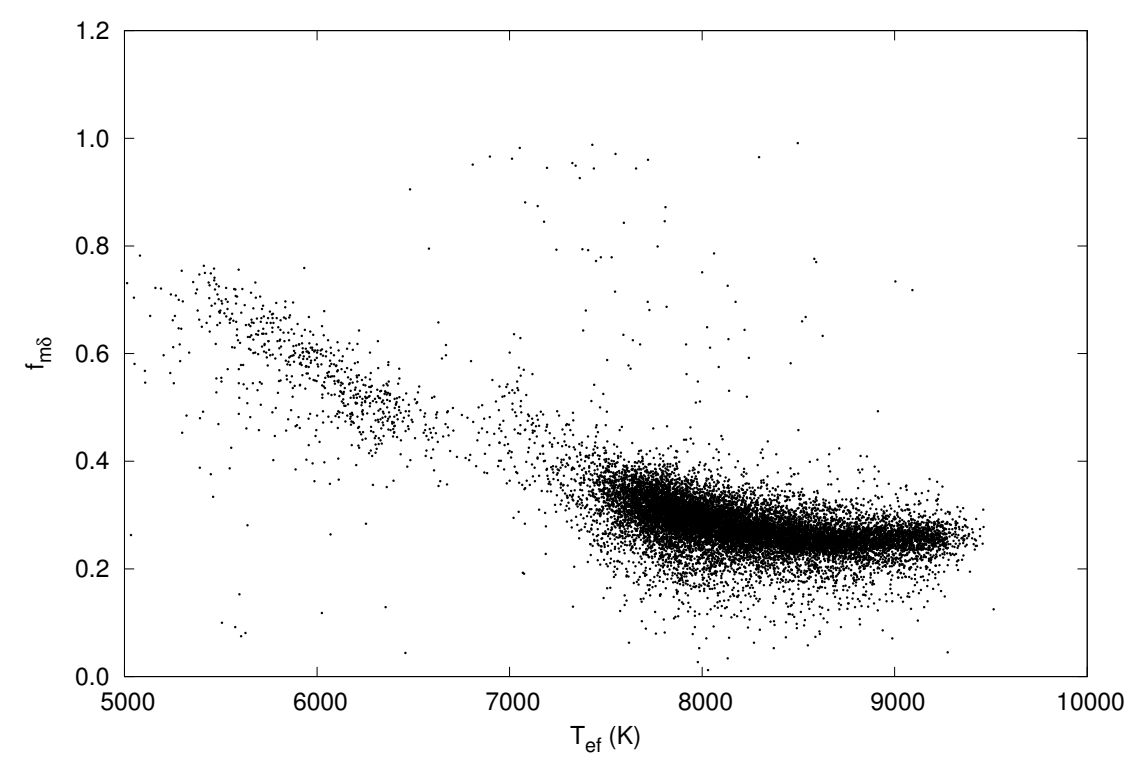

Figura 3.15: Relação entre os parâmetros $\mathrm{T}_{e f}$ vs $\mathrm{f}_{m \delta}$. Nota-se que há uma nítida correlação entre a temperatura efetiva estimada pelo SSPP e o parâmetro relacionado com a medida do fluxo no centro da linha em relação ao contínuo.

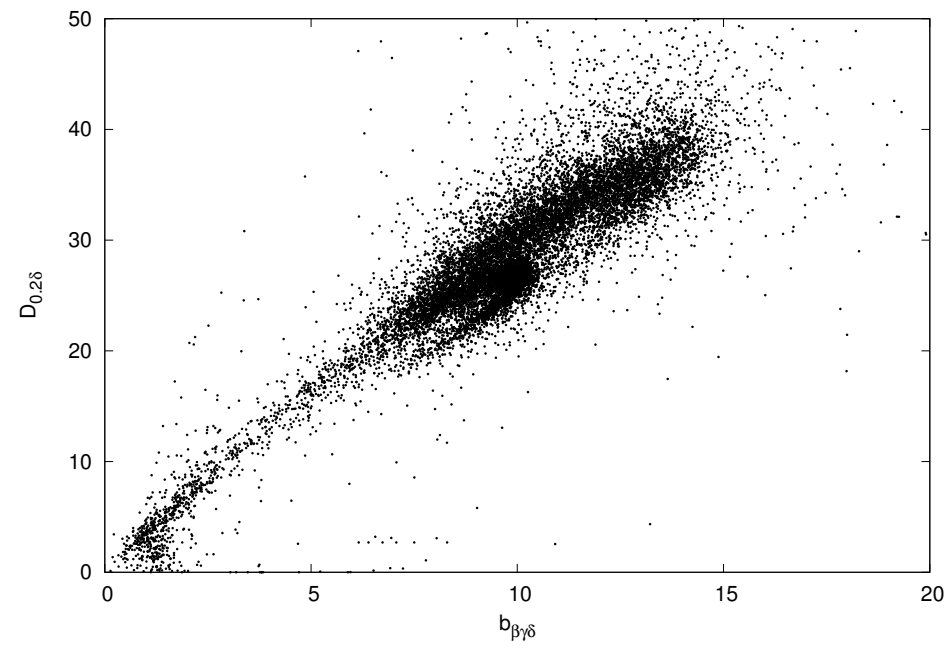

Figura 3.16: Relação entre os parâmetros $\mathrm{D}_{0.2 \delta}$ e $b_{\beta \gamma \delta}$. Há uma nítida correlação entre ambos. Entretanto, a comparação entre eles não revela diferença entre estrelas anãs e gigantes.

relação empírica entre a linha K do Ca II (3933.7 ^) e a metalicidade descrita inicialmente por Beers et al. (1990) e revisada por Beers et al. (1999). O método estima a metalicidade através do cálculo da pseudo-largura equivalente da linha $\mathrm{K}$ do CaII para os intervalos: $-4.0 \leq[\mathrm{Fe} / \mathrm{H}] \leq 0.5$ e $0.3 \leq(B-V) \leq 1.2$. No capítulo seguinte mostra-se que as 


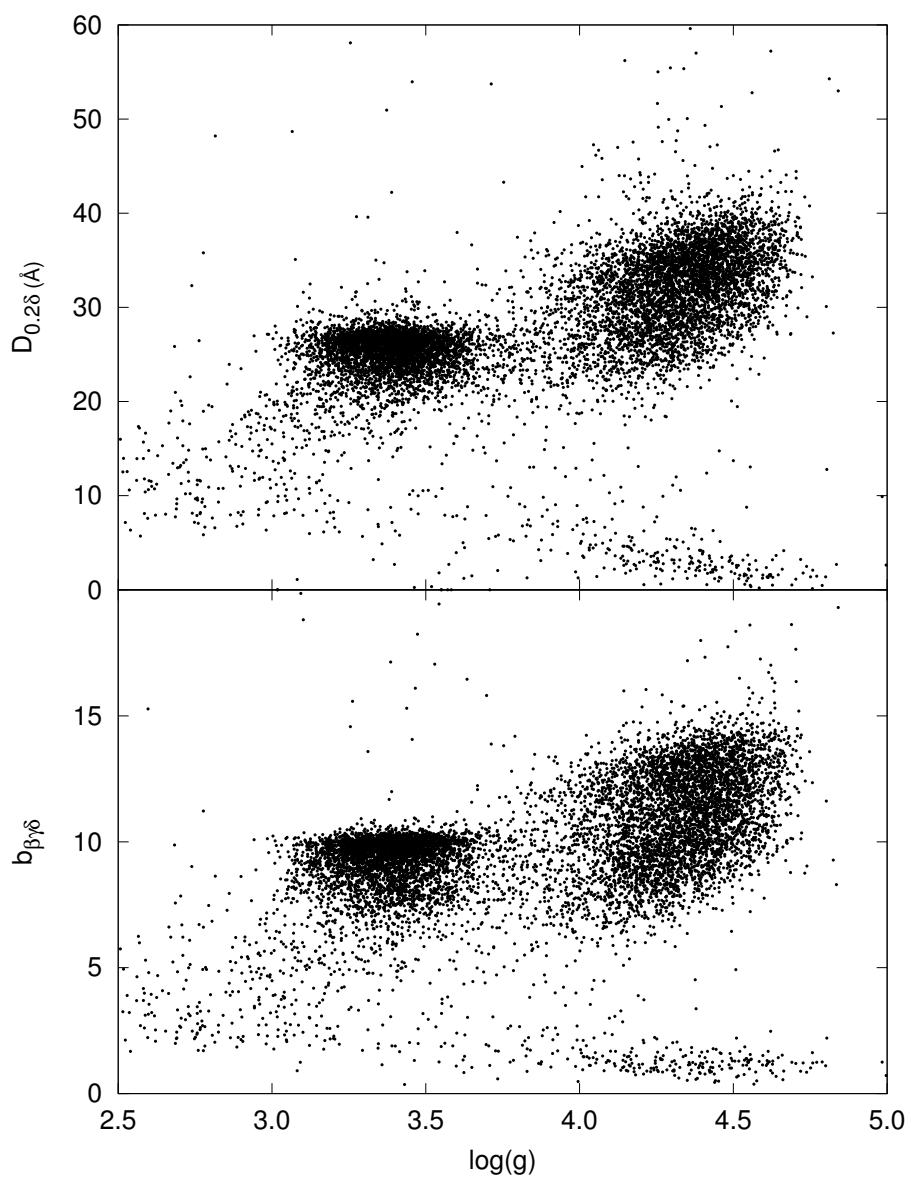

Figura 3.17: Relação entre os parâmetros $\log (\mathrm{g})$ vs $b_{\beta \gamma \delta}$ (painel inferior) e $\log (\mathrm{g})$ vs $\mathrm{D}_{0.2 \delta}$ (painel superior). Não há correlação entre os parâmetros comparados.

estrelas BS selecionadas possuem índice $(B-V)<0.3$. Através da Figura 3.18 verifica-se que, quanto menor a metalicidade estelar, menos intensa é a linha K do Ca II, de modo que, mesmo para estrelas com temperaturas efetivas acima de $7500 \mathrm{~K}$, as estimativas de $[\mathrm{Fe} / \mathrm{H}]$ dadas pelo SSPP se mostram coerentes e possuem erro médio de 0.2 dex para estrelas com $g<18$ (razão $\langle\mathrm{S} / \mathrm{R}\rangle$ maior).

\subsection{Estrelas com magnitude $g>18$}

Diante da limitação dos métodos tradicionais em avaliar estrelas com $g>18$ em vista das baixas razões $\langle$ S/R $\rangle$ (Clewley et al., 2002; Sirko et al., 2004), o SSPP é a uma ferramenta que está disponível e é capaz de avaliar a contaminação de estrelas gigantes para magnitudes elevadas. Como exemplificado na Figura 3.19, mesmo que o perfil de Sérsic estime os 


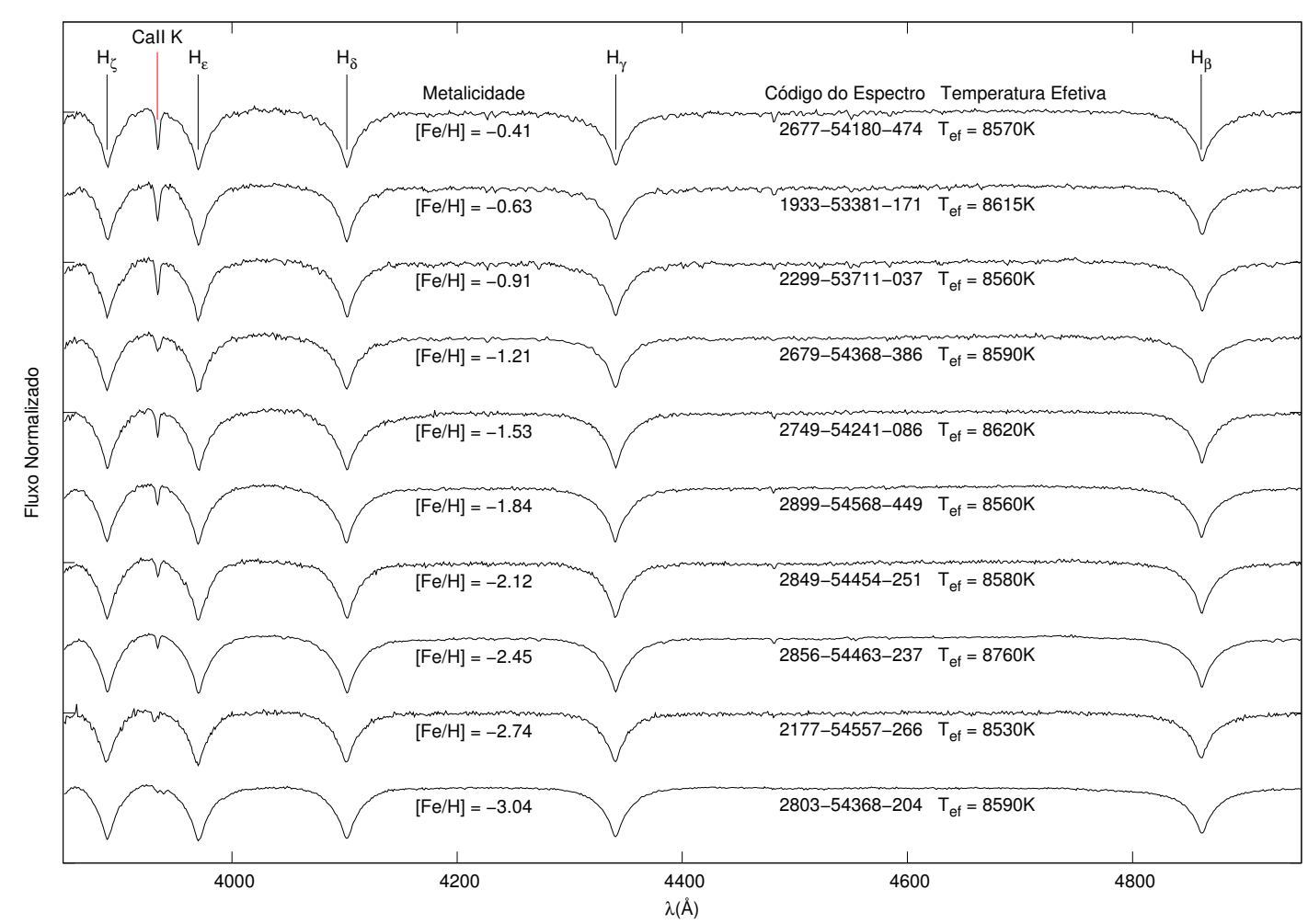

Figura 3.18: Relação entre a intensidade da linha K do Ca II (3933.7 ̊) e a metalicidade estimada pelo SSPP. A relação mostra uma tendência coerente mesmo para estrelas com temperatura efetiva acima de $7500 \mathrm{~K}$, pois quanto menor a metalicidade, menor será a pseudo largura equivalente da linha do Cálcio.

parâmetros dos métodos clássicos para $\langle\mathrm{S} / \mathrm{R}\rangle \sim 9$, a distinção entre as populações estelares começa a se dificultar para $g>18$.

A inclusão de objetos com magnitudes $g>18$ é fundamental, pois as estrelas anãs são frequentes nesse intervalo (Deason et al., 2011). Devido ao limite observacional de estrelas BHB na Galáxia (como mostrado na Figura 3.20), a proporção de estrelas gigantes diminui bruscamente com o aumento da magnitude. Ao atingir $g>17$, o número de estrelas de tipo A com $\log (\mathrm{g})$ típicos de gigantes é menor que o número de estrelas anãs. Para magnitudes $g>19$, a fração de estrelas gigantes é quase inexistente, o que torna a inclusão de estrelas de sequência principal nesse intervalo de magnitude ainda mais interessante, pois a contaminação de estrelas BHB é baixa.

A distribuição de $\log (\mathrm{g})$ para estrelas com magnitudes $g>18$ é apresentada na Figura 3.21. São 5663 estrelas que podem ser avaliadas pelo SSPP e seriam descartadas pelos métodos tradicionais. A Figura 3.21 mostra um pico centrado em valores de $\log (\mathrm{g})=$ 

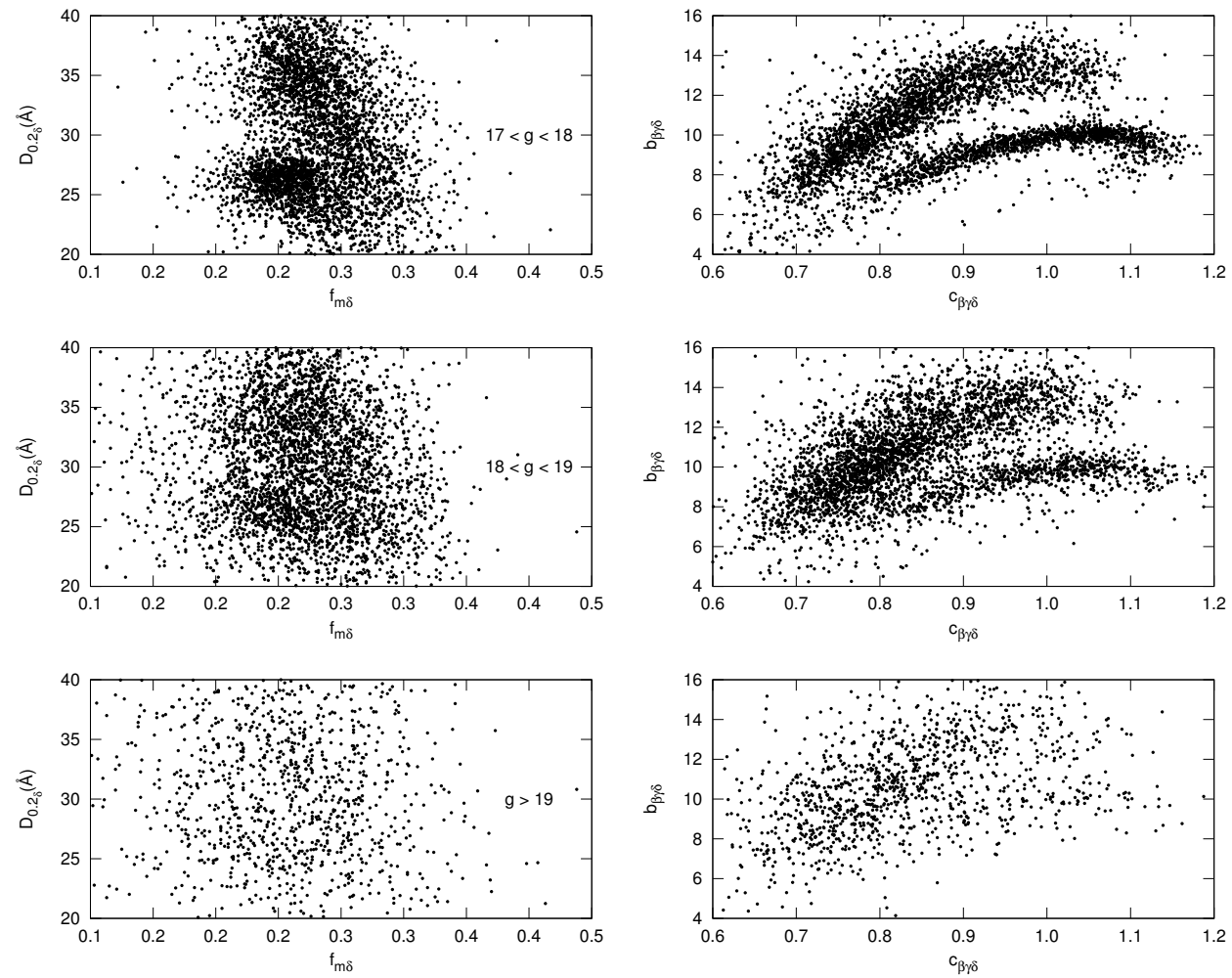

Figura 3.19: Limitação do método $\mathrm{f}_{m \delta}$ vs $\mathrm{D}_{0.2 \delta}$ para magnitude $\mathrm{g}>18$ (painéis esquerdos). A mesma dificuldade de separação entre estrelas anãs e gigantes ocorre para o método $c_{\beta \gamma \delta}$ vs $b_{\beta \gamma \delta}$ (painéis direitos), nos mesmos intervalos de magnitudes.

3.573, típico de estrelas gigantes e subgigantes com alta dispersão $(\sigma=0.328)$, a qual torna impossível considerar a região acima de $3 \sigma$ como limitante para estrelas BS. Nesse caso, as contaminações inevitavelmente são maiores e são avaliadas a cada $\sigma$, como pode ser visto na Figura 3.22, em relação a $\log (\mathrm{g})$ e à temperatura efetiva $\left(\sigma_{T_{\text {ef }}}=157 \mathrm{~K}\right)$.

\subsection{Combinação de métodos}

Para selecionar as estrelas BS com magnitudes $g<18$, combina-se os métodos $c_{\beta \gamma \delta}$ vs $b_{\beta \gamma \delta}$ com as restrições adotadas pelo SSPP. Inicialmente, uma região limite para as estrelas BHB no método de forma e largura deve ser definida. Para tanto, uma função polinomial de quarta ordem foi ajustada sobre o ramo da distribuição classificado como gigante (Figura 3.23). Dessa maneira, a margem de erro da região foi avaliada através dos resíduos do ajuste (Figura 3.24). A combinação dos métodos, o ajuste e sua margem de erro são mostrados na Figura 3.23. 


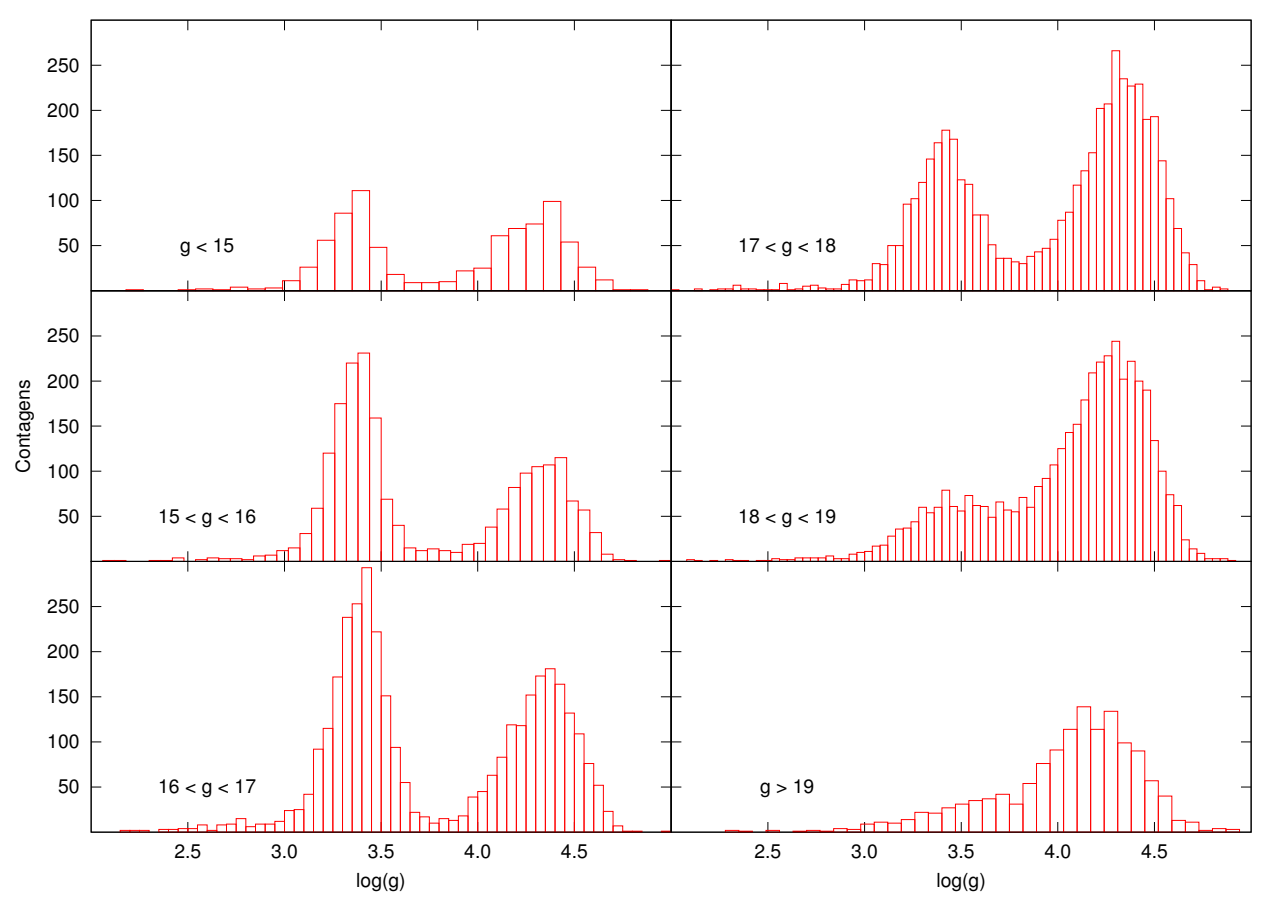

Figura 3.20: Limite de magnitude aparente para estrelas BHB da Galáxia. A proporção de estrelas no pico com $\log (\mathrm{g})$ típico de gigante começa a diminuir drasticamente para magnitude $g \sim 18$.

No caso de combinação de restrições para as estrelas com magnitudes $g<18$, os objetos remanescentes desses métodos possuem confiabilidade média maior que $99 \%$ de serem anãs na sequência principal, e aliadas a baixa metalicidade já definidas, são vistas como as melhores candidatas à azuis tardias (BS). O erro relacionado com a temperatura efetiva adotado foi o erro médio do SSPP $(157 \mathrm{~K})$. Na Figura 3.25, o retângulo destaca a região de maior confiabilidade de estrelas BS.

As estrelas com $g<18$ possuem erro fotométrico médio de $0.22 \%$ e $\sigma_{g_{<18}}=0.138$. Para $g>18$, os valores são maiores: erro fotométrico $=0.53 \%$ e $\sigma_{g>18}=0.328$. As tabelas 3.1 e 3.2 resumem os erros dos métodos em todas as etapas e mostram a confiabilidade e a quantidade de estrelas a cada $\sigma$, respectivamente.

\subsection{Discussão}

A seleção de estrelas BS através dos métodos combinados possibilitou identificar estrelas BS (para $g<18$ ) com mais de $97 \%$ de confiança. Além disso, o SSPP permitiu expandir 
Tabela 3.1 - Confiabilidade na seleção da amostra de estrelas BS (expressa em \%).

\begin{tabular}{c|c|c|c|c}
\hline BS: Confiança & $\log (\mathrm{g})>3 \sigma$ & $1 \sigma<\log (\mathrm{g})<2 \sigma$ & $\log (\mathrm{g})>2 \sigma$ & - \\
$\mathrm{T}_{e f}(\mathrm{~K})$ & $g<18$ & $g>18$ & $g>18$ & $\langle$ Confiança $\rangle$ \\
\hline $\mathrm{T}_{e f}<1 \sigma_{T_{e f}}$ & $65.90 \%$ & $63.30 \%$ & $65.80 \%$ & $64.76 \%$ \\
$1 \sigma_{T_{e f}}<\mathrm{T}_{e f}<2 \sigma_{T_{e f}}$ & $86.40 \%$ & $80.80 \%$ & $86.20 \%$ & $85.07 \%$ \\
$2 \sigma_{T_{e f}}<\mathrm{T}_{e f}<3 \sigma_{T_{e f}}$ & $97.90 \%$ & $86.20 \%$ & $97.00 \%$ & $95.41 \%$ \\
$\mathrm{~T}_{e f}>3 \sigma_{T_{e f}}$ & $99.70 \%$ & $86.40 \%$ & $97.80 \%$ & $97.29 \%$ \\
\hline Total & $96.59 \%$ & $82.85 \%$ & $94.68 \%$ & $93.65 \%$ \\
\hline
\end{tabular}

Tabela 3.2 - Número de estrelas presentes em cada intervalo de erro da Tabela 3.1.

\begin{tabular}{c|c|c|c|c}
\hline BS: Quantidade & $\log (\mathrm{g})>3 \sigma$ & $1 \sigma<\log (\mathrm{g})<2 \sigma$ & $\log (\mathrm{g})>2 \sigma$ & - \\
$\mathrm{T}_{e f}(\mathrm{~K})$ & $g<18$ & $g>18$ & $g>18$ & Soma \\
\hline $\mathrm{T}_{e f}<1 \sigma_{T_{e f}}$ & 250 & 180 & 93 & 523 \\
$1 \sigma_{T_{e f}}<\mathrm{T}_{e f}<2 \sigma_{T_{e f}}$ & 685 & 311 & 305 & 1301 \\
$2 \sigma_{T_{e f}}<\mathrm{T}_{e f}<3 \sigma_{T_{e f}}$ & 811 & 315 & 424 & 1550 \\
$\mathrm{~T}_{e f}>3 \sigma_{T_{e f}}$ & 3092 & 814 & 1244 & 5150 \\
\hline Total & 4838 & 1620 & 2066 & 8524 \\
\hline
\end{tabular}




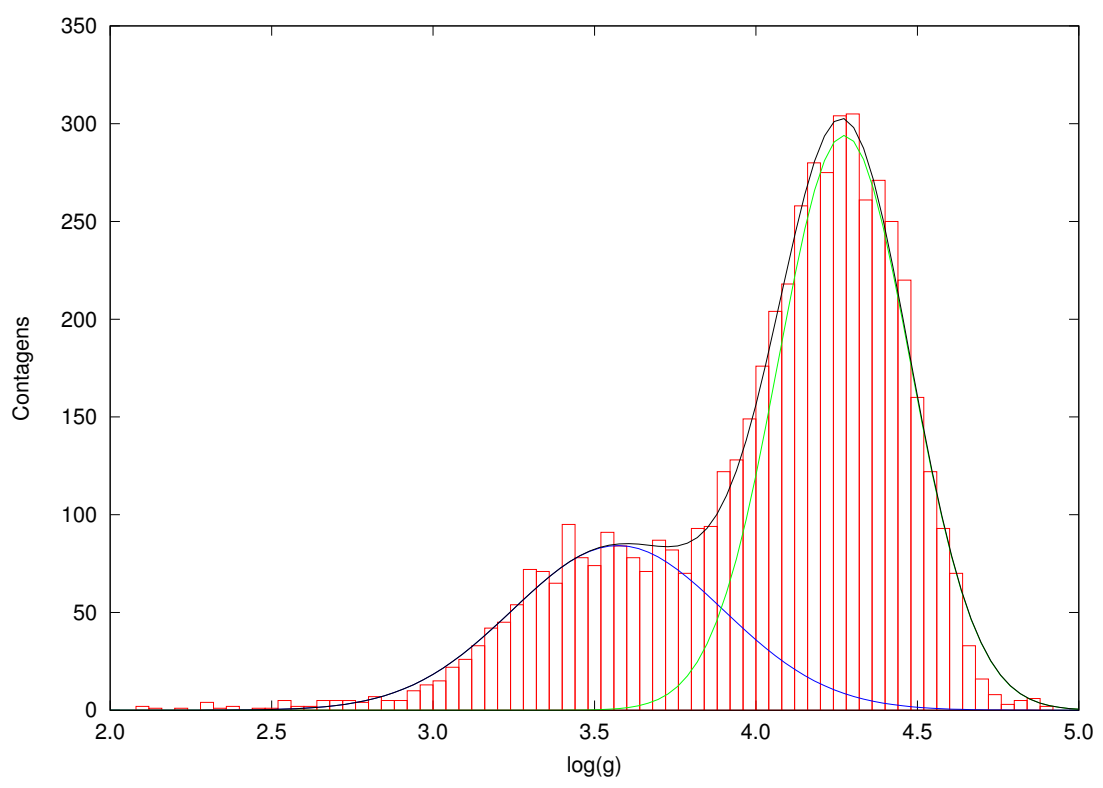

Figura 3.21: Distribuição de $\log (\mathrm{g})$ (para magnitudes $g>18$ ) de parâmetros gerados pelo SSPP. O pico centrado em $\log (\mathrm{g})=3.573(\sigma=0.328)$ representa a distribuição de estrelas gigantes e subgigantes e $\log (\mathrm{g})=4.274(\sigma=0.204)$ se aplica às estrelas anãs na sequência principal.

a amostra incluindo objetos com $g>18$. Entretanto, levando-se em conta maiores erros derivados das baixas razões $\langle\mathrm{S} / \mathrm{R}\rangle$, a confiança diminui para $\sim 85 \%$.

As relações entre os parâmetros do SSPP e dos métodos clássicos $\left(\mathrm{f}_{m \delta}\right.$ vs $\mathrm{D}_{0.2 \delta}$ e $c_{\beta \gamma \delta}$ vs $\left.b_{\beta \gamma \delta}\right)$ merecem ser revistas quantitativamente, principalmente a relação entre $\mathrm{f}_{m}$ e $\mathrm{T}_{e f}$, a qual fornece uma calibração útil dos parâmetros estelares. Além disso, elas também podem ser adotadas como novas alternativas às rotinas internas do SSPP. O mesmo vale para o método de escala de largura e forma, e para os critérios restritivos, descritos ao longo desse capítulo, que avaliam a separação entre anãs e gigantes pelo SSPP.

As estrelas presentes nas faixas de confiabilidade maiores que 97\% totalizam 5571 objetos, cuja confiabilidade média é de $98.81 \%$. As estrelas presentes nas faixas de confiabilidade menores que $97 \%$ e maiores que $80 \%$ somam 2430 objetos, cuja confiabilidade média é de $85.63 \%$. A amostra final conta com essas duas regiões, que totalizam 8001 estrelas BS, cuja confiabilidade média é de $94.80 \%$. No próximo capítulo são apresentadas as estimativas de distâncias e distribuições de velocidades para esses objetos. 


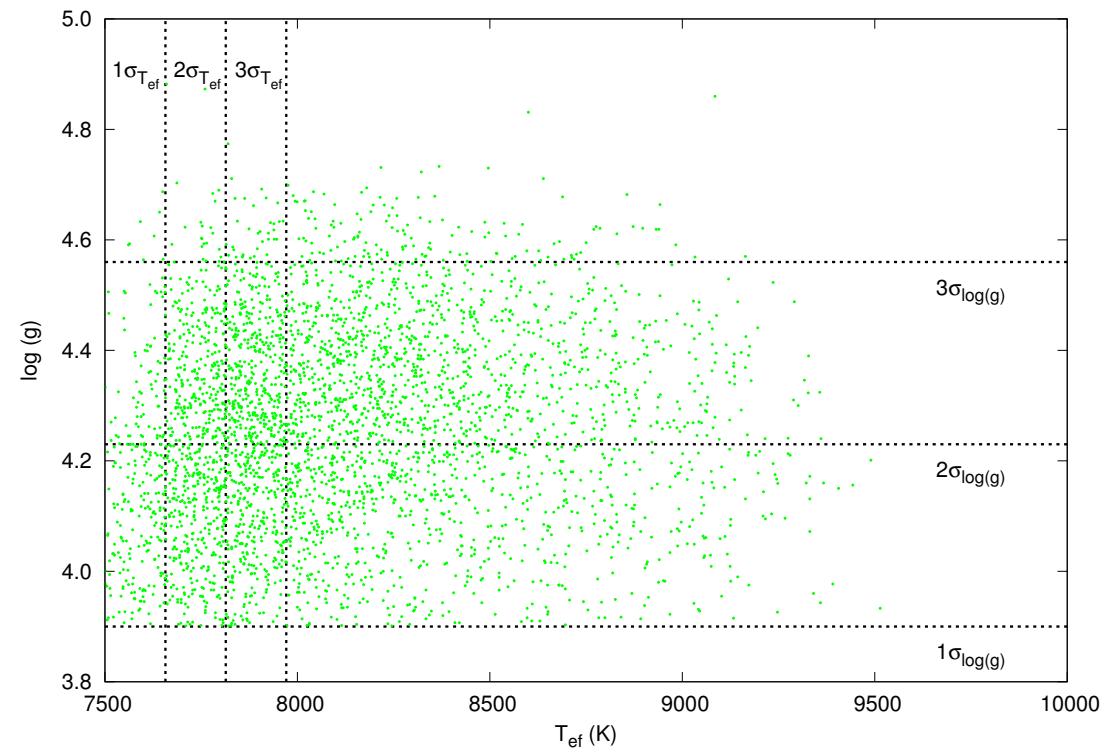

Figura 3.22: Parâmetros atmosféricos estelares gerados pelo SSPP para magnitude $g>18$. A incerteza na temperatura efetiva dada pelo SSPP é $\sigma_{T_{e f}}=157 \mathrm{~K}$ e a distribuição de $\log (\mathrm{g})$ tem $\sigma_{\log (g)}=0.328$. Ao contrário do critério adotado para estrelas com magnitude $g<18$, poucos objetos se encotram na região acima de $3 \sigma_{\log (g)}$. Nesse caso, a confiança na seleção é avaliada a cada $\sigma_{\log (g)}$, como mostram as Tabelas 3.2 e 3.1 . 


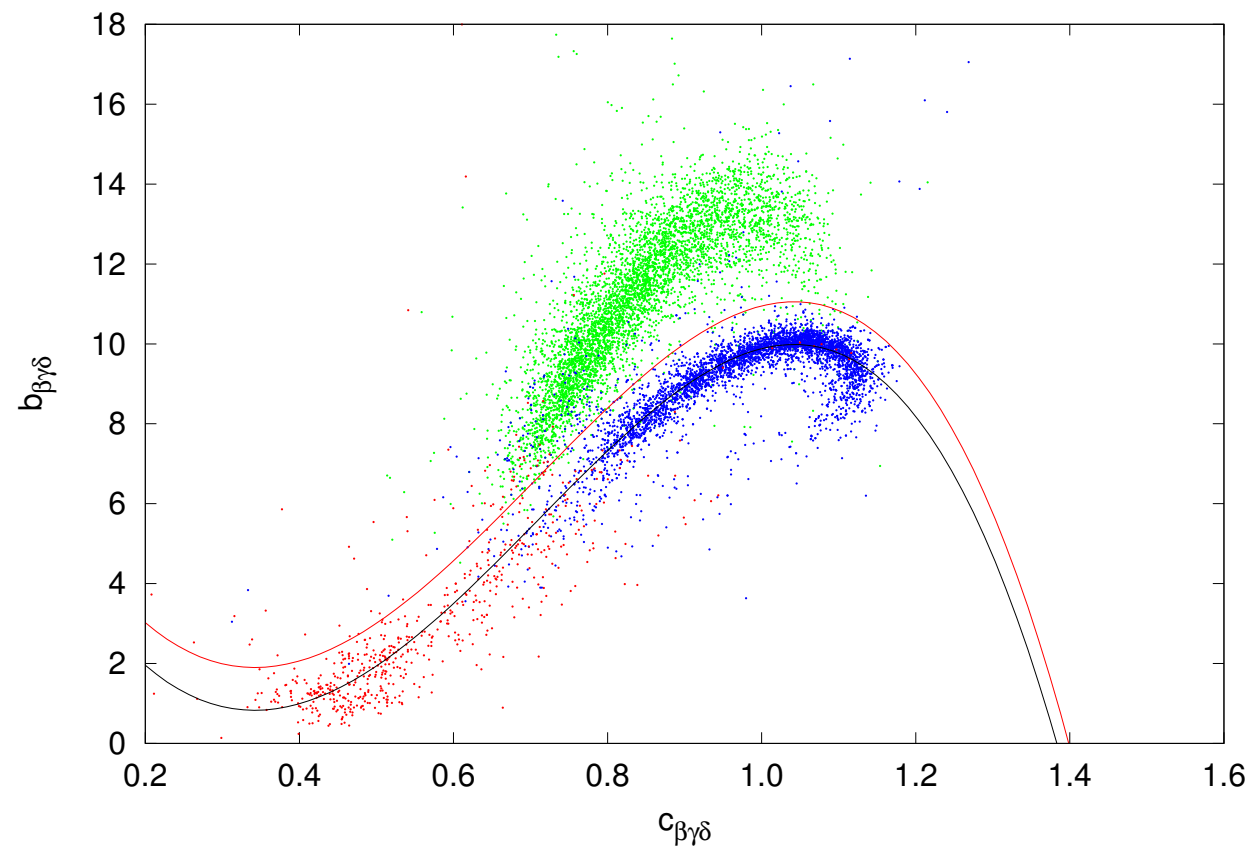

Figura 3.23: Parâmetros $c_{\beta \gamma \delta}$ vs $b_{\beta \gamma \delta}$ para estrelas com magnitude $g<18$. A figura foi colorida de acordo com os limites de separação de estrelas anãs adotados para o método do SSPP: estrelas BHB em azul, BS em verde e estrelas de sequência principal mais frias (F, G e K) em vermelho. A linha preta representa o ajuste polinomial de quarta ordem e a linha vermelha sua margem de erro $(3 \sigma=1.07)$.

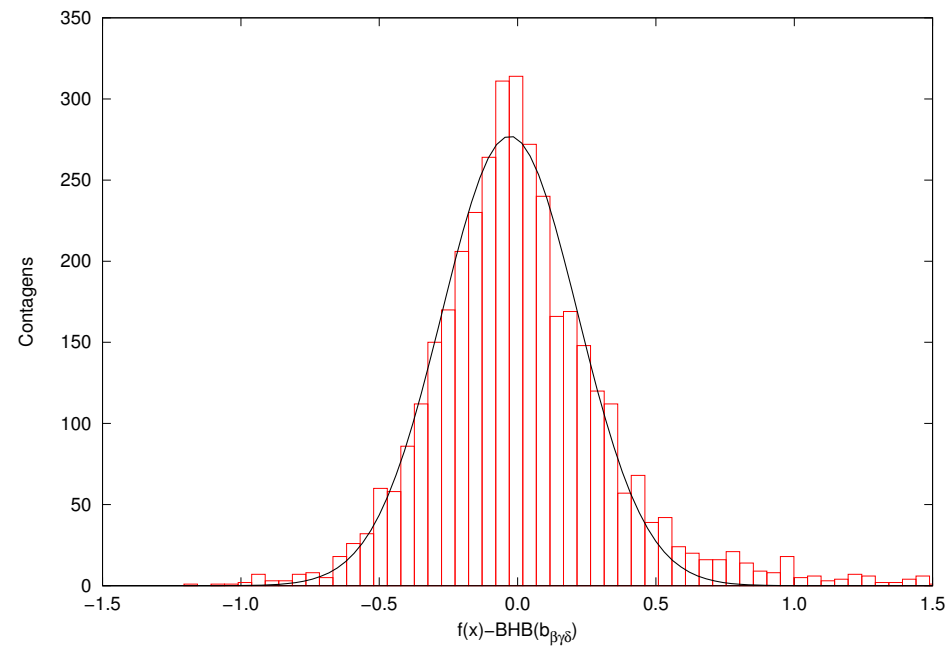

Figura 3.24: Resíduos do ajuste polinomial de ordem 4 sobre a região BHB $(g<18)$. $\langle\mathrm{x}\rangle=$ -0.028 e $\sigma=0.345$. 


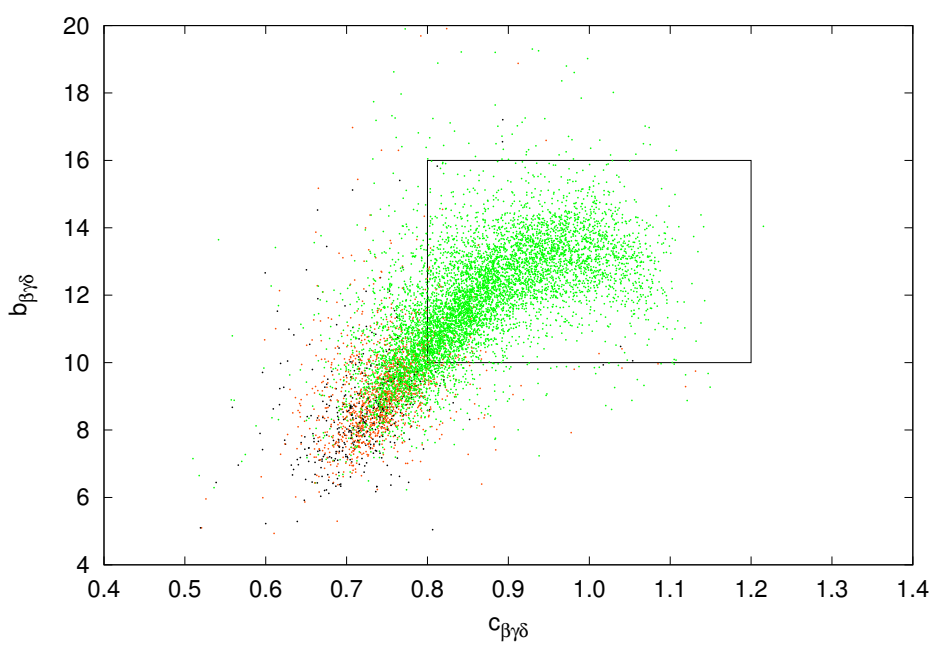

Figura 3.25: Estrelas que foram classificadas como anãs por todos os métodos. Os pontos estão coloridos segundo a margem de erro de temperatura efetiva $\left(\sigma_{T_{e f}}=157 \mathrm{~K}\right)$. As estrelas com $\mathrm{T}_{e f}<7657 \mathrm{~K}$ são identificadas por pontos pretos, em laranja estão os objetos entre 7657 $\mathrm{K}<\mathrm{T}_{e f}<7814 \mathrm{~K}$ e em verde as estrelas com $\mathrm{T}_{e f}>7814 \mathrm{~K}$, que são as melhores candidatas a estrelas BS, destacadas dentro do retângulo. 
Capítulo 4

\section{Cinemática das Estrelas BS}

Neste capítulo são apresentadas as relações utilizadas para estimar as magnitudes absolutas das estrelas BS, assim como as distâncias até elas. Em seguida, são avaliadas as distribuições de posições, metalicidades e velocidades, com a finalidade de identificar grupos distintos desses objetos.

\subsection{Estimativas de magnitude absoluta e distâncias}

As 8001 estrelas BS selecionadas pelos métodos descritos no capítulo anterior possuem magnitudes aparentes entre $12<g<20$. Entretanto, trabalhos tradicionais (Kinman et al., 1994; Preston et al., 1994; Preston e Sneden, 2000; Carney et al., 2001, 2005) geralmente utilizam calibrações de magnitudes absolutas adotando sistemas fotométricos diferentes do ugriz do SDSS. Assim, a compatibilidade entre os diferentes sistemas fotométricos é importante para comparação entre dados de estrelas BS encontrados na literatura disponível. Zhao e Newberg (2006) derivaram uma transformação de magnitudes absolutas relacionando estrelas do SDSS encontradas no survey HK (Beers et al., 1985, 1992), com magnitudes no sistema Johnson $(U B V)$, obtendo:

$$
\begin{gathered}
V=g-0.561 \cdot(g-r)-0.004, \\
(B-V)=0.916 \cdot(g-r)+0.187 .
\end{gathered}
$$

O intervalo de transformações é válido para $-0.5<(g-r)<1.0$ (Beers et al., 2012), sendo coerente com os índices de cor adotados no ínicio da seleção de objetos neste trabalho. 
A partir da magnitude aparente na banda $V$, a magnitude absoluta pode ser estimada pela relação proposta por Kinman et al. (1994), através da Equação 4.3 e, na banda $g$, pela Equação 4.4 descrita por Deason et al. (2011).

\subsubsection{Magnitude absoluta $M_{V}$}

Kinman et al. (1994) adotaram uma estimativa de magnitude absoluta baseada em estrelas BS de aglomerados globulares (Sarajedini, 1993, 1994). Admitindo $(B-V)=0.1$ e $[\mathrm{Fe} / \mathrm{H}]=-1.7$, típicos das estrelas A pobres em metais selecionadas neste trabalho, os autores verificaram que as estrelas BS de campo possuem magnitude absoluta na banda $V$ distribuídas em torno de 2.49, utilizando a relação:

$$
M_{V}=1.32+4.05 \cdot(B-V)-0.45 \cdot[F e / H] .
$$

A Figura 4.1 mostra o diagrama cor-magnitude da amostra utilizada neste trabalho. Estrelas com $[\mathrm{Fe} / \mathrm{H}]>-2.0$ estão representadas em azul e as estrelas com $[\mathrm{Fe} / \mathrm{H}]<-2.0$ em vermelho.

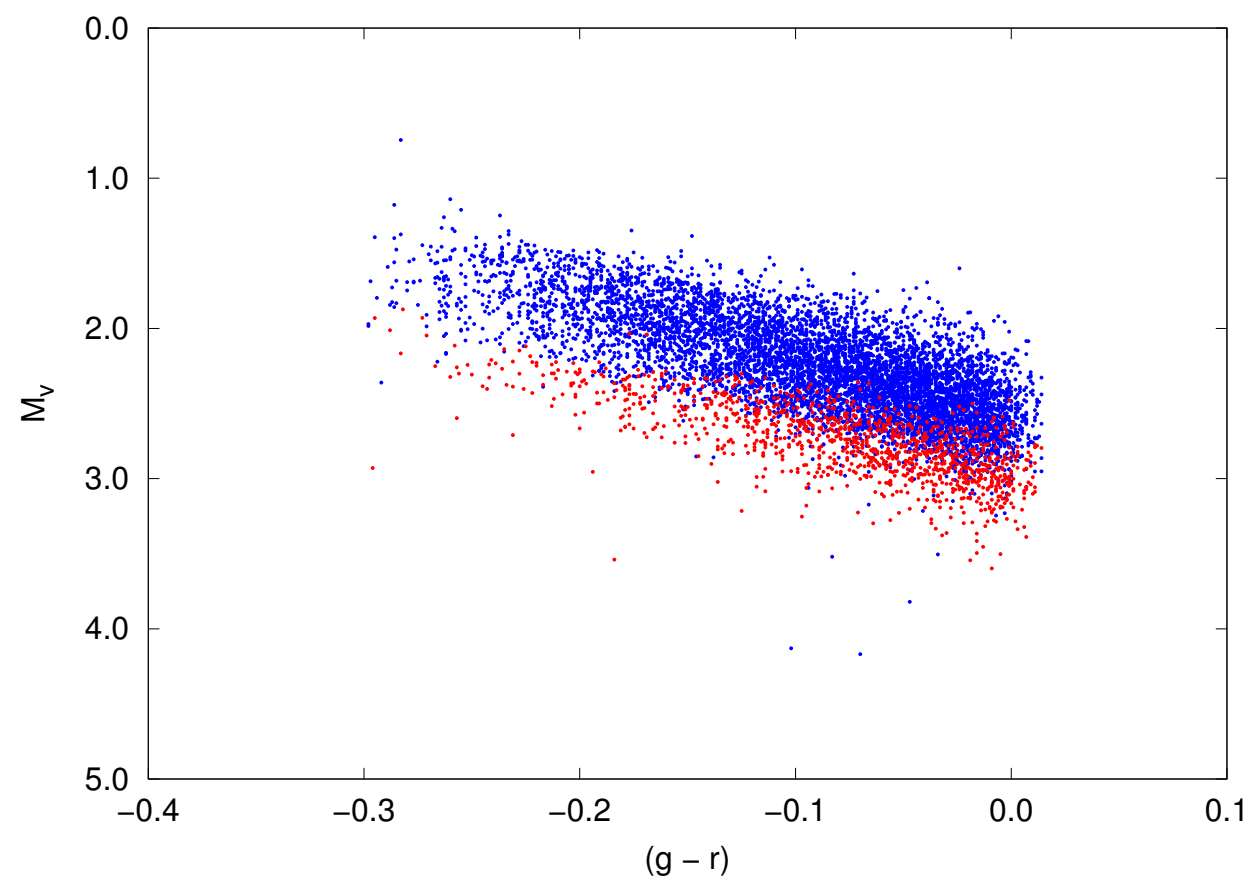

Figura 4.1: Diagrama cor-magnitude construído com o índice de cor do SDSS e a estimativa de magnitude de Kinman et al. (1994). Os pontos em vermelhos representam as estrelas com $[\mathrm{Fe} / \mathrm{H}]<-2.0$ e em azul com $[\mathrm{Fe} / \mathrm{H}]>-2.0$. 


\subsubsection{Magnitude absoluta $M_{g}$}

As estrelas gigantes são intrinsicamente mais brilhantes que as estrelas de sequência principal e suas magnitudes absolutas variam pouco com a metalicidade (Deason et al., 2011). Entretanto, as estrelas BS são menos luminosas e se espalham por um intervalo grande de magnitudes absolutas (Figura 4.2). Além disso, elas são menos frequentes em aglomerados globulares que as estrelas BHB. Baseados nesses fatos, Deason et al. (2011) adotaram uma calibração para magnitude absoluta na banda $g$ utilizando estrelas BS da corrente de Sagitário, na região entre $25^{\circ}<\alpha<40^{\circ}$. Watkins et al. (2009) usaram RR Lyræ para estimar a distância até a corrente como sendo $\mathrm{D}_{S g r}=26.1 \pm 5.6 \mathrm{kpc}$. A calibração de magnitude absoluta $\left(M_{g}\right)$ é similar à Kinman et al. (1994) para $[\mathrm{Fe} / \mathrm{H}]=-1.5$, e também concorda com a metalicidade da corrente encontrada por Watkins et al. (2009) $([\mathrm{Fe} / \mathrm{H}]=-1.43)$. Entretanto, Deason et al. (2011) não encontraram forte dependência com a metalicidade para sua calibração de magnitude absoluta e, seu erro, $\sigma_{g(B S)}=0.5$, é baseado principalmente no erro da distância até a corrente de Sagitário. A relação de calibração proposta por Deason et al. (2011) é:

$$
M_{g(B S)}=3.108+5.495 \cdot(g-r) .
$$

As magnitudes absolutas encontradas em ambas as bandas se distribuem em torno de 2.5 (Figuras 4.1 e 4.2). As distâncias até os objetos foram calculadas segundo a expressão:

$$
D=10\left[\left(\frac{m-M+5}{5}\right)-3\right](\mathrm{kpc})
$$

e são sistematicamente menores para o método que utiliza como referência as estrelas da corrente de Sagitário (Figura 4.3) devido à independência da metalicidade. Assim, ao longo do trabalho, a estimativa de magnitude absoluta utilizada para avaliar a distribuição das estrelas BS na Galáxia é a encontrada para a banda $V$.

O erro na estimativa de magnitude absoluta na banda $V$ leva em consideração os erros propagados na metalicidade e no índice de cor. Como consequência direta, os erros são menores para estrelas com magnitudes aparentes menores (cuja fotometria é melhor) e são maiores para estrelas distantes, sendo mais razoáveis que o erro adotado pelo método na banda $g$ (constante em $0.5 \mathrm{mag}$ ). 


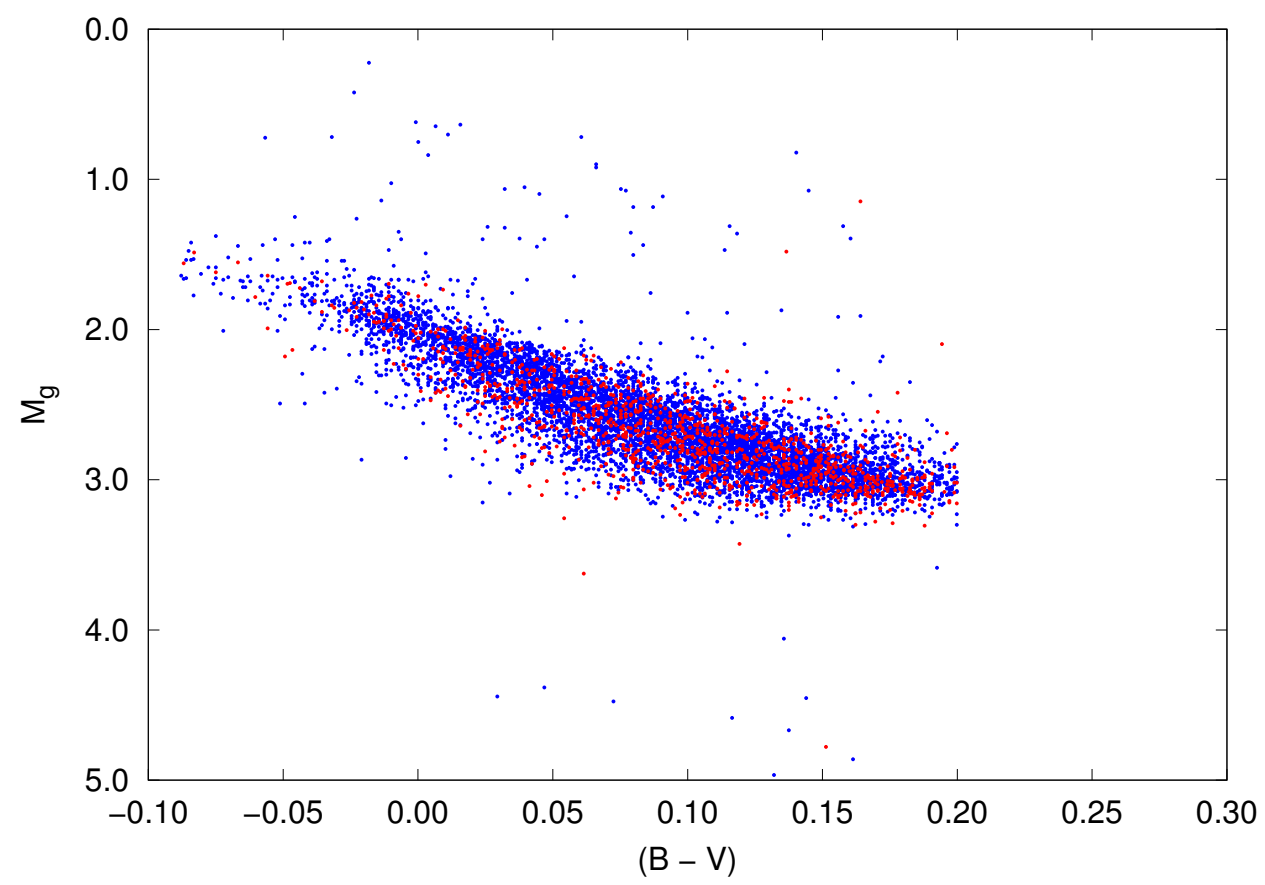

Figura 4.2: Diagrama cor-magnitude construído com o índice de cor $(B-V)$ e a estimativa de magnitude absoluta de Deason et al. (2011). Os pontos vermelhos representam estrelas com $[\mathrm{Fe} / \mathrm{H}]<-2.0$ e em azul com $[\mathrm{Fe} / \mathrm{H}]>-2.0$.

Como forma de avaliar os índices de cor $(B-V)$ determinados anteriormente, a Figura 4.4 apresenta as estrelas BS selecionadas neste trabalho no limite descrito por Carney et al. (2005) na Figura 1.4. Elas se encontram, como esperado, na região acima do limite de cor definido pela média dos pontos de turnoff de aglomerados globulares.

As Figuras 4.5 e 4.6 mostram, respectivamente, as distâncias encontradas por Deason et al. (2011) e as distâncias estimadas para a amostra selecionada. Vê-se que elas concordam com as distâncias de estrelas BS de campo estimadas por Yanny et al. (2000); Clewley et al. (2004) e que a maioria encontra-se a distâncias de até $30 \mathrm{kpc}$.

Pode-se calcular as posições das estrelas nas coordenas X, Y e Z (Murray, 1983) utilizando-se as distâncias calculadas e as coordenadas de longitude $(l)$ e latitude $(b)$ galácticas:

$$
\begin{gathered}
X=R_{\odot}-R \cos b \cos l, \\
Y=R \cos b \sin l, \\
Z=R \sin b,
\end{gathered}
$$




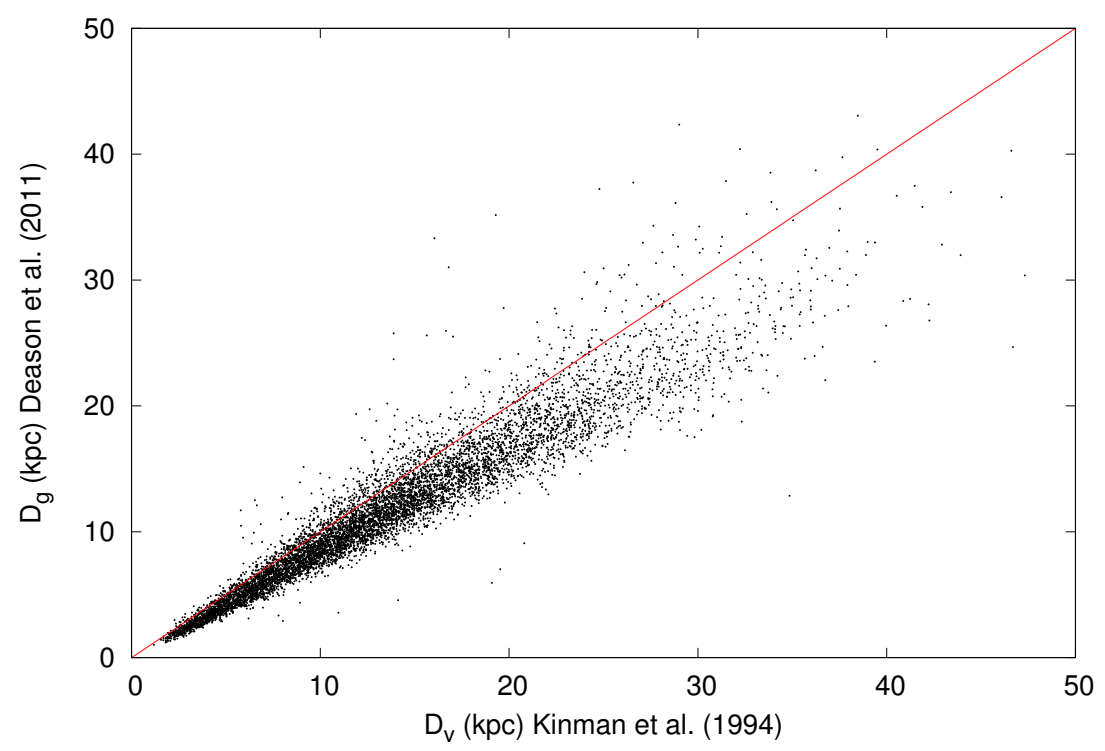

Figura 4.3: Comparação entre as distâncias estimadas pelo método de Kinman et al. (1994) e Deason et al. (2011). Eles adotam como referência, respectivamente, estrelas BS em aglomerados globulares e na corrente de Sagitário. As distâncias estimadas através da magnitude $g$ são sistematicamente menores pois desconsideram a dependência de $[\mathrm{Fe} / \mathrm{H}]$.

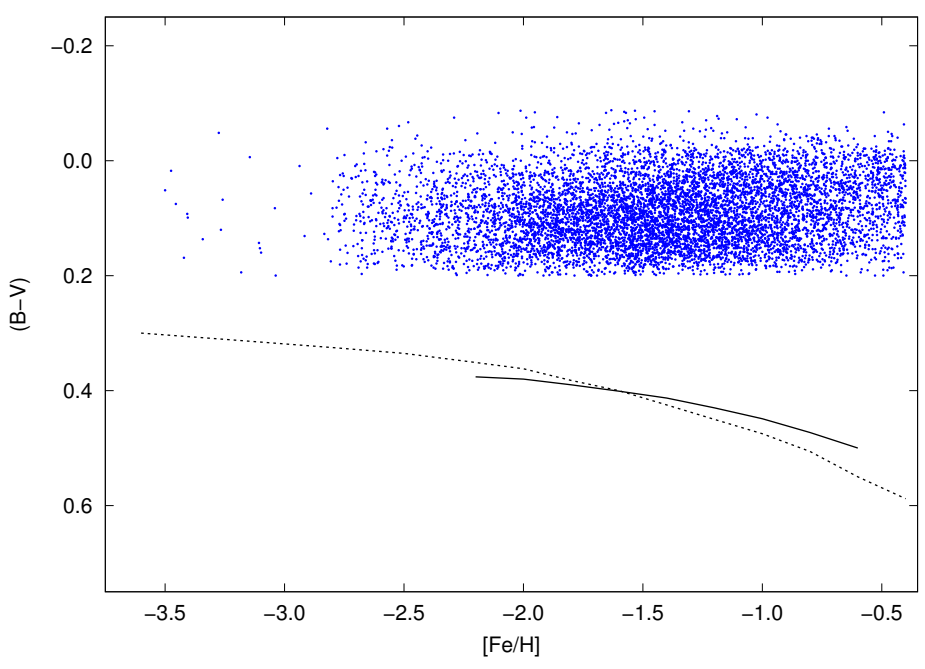

Figura 4.4: Magnitudes absolutas na banda $V$ vs $[\mathrm{Fe} / \mathrm{H}]$ para as estrelas BS selecionadas. Assim como mostrou a Figura 1.4, a linha tracejada caracteriza o limite teórico para as estrelas BS em aglomerados globulares (através do ponto de turnoff da sequência principal), enquanto a linha contínua representa o mesmo limite considerando-se dados observacionais.

onde a distância do Sol ao centro galáctico $\left(R_{\odot}\right)$ é adotada como sendo 8 kpc (Reid, 1993). A distribuição das estrelas BS na Galáxia é mostrada na Figura 4.7. O plano XY contém 


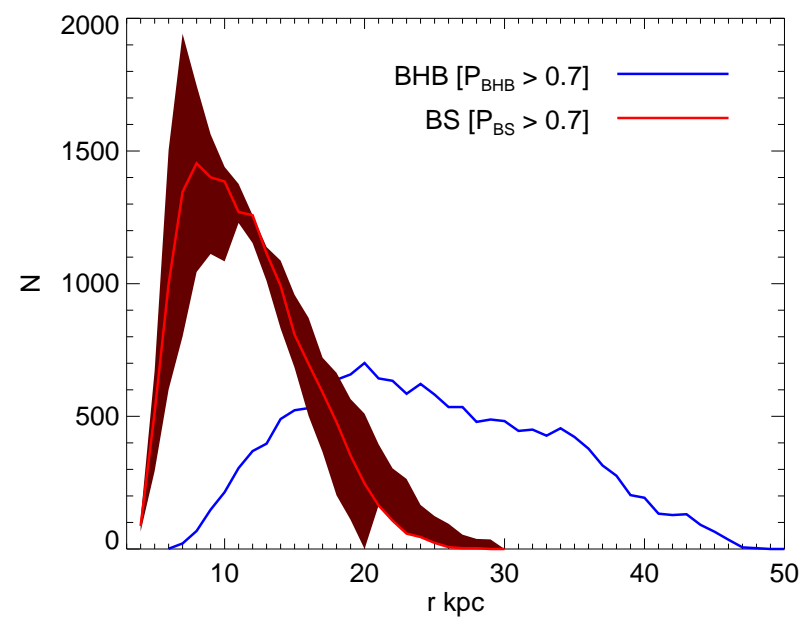

Figura 4.5: Distribuição de distâncias para estrelas BS e BHB. A linha em azul mostra a região onde a probabilidade de encontrar uma estrela BHB é maior que $70 \%$, a mesma probabilidade é vista para a linha vermelha sombreada, com estrelas BS. Figura retirada de Deason et al. (2011).

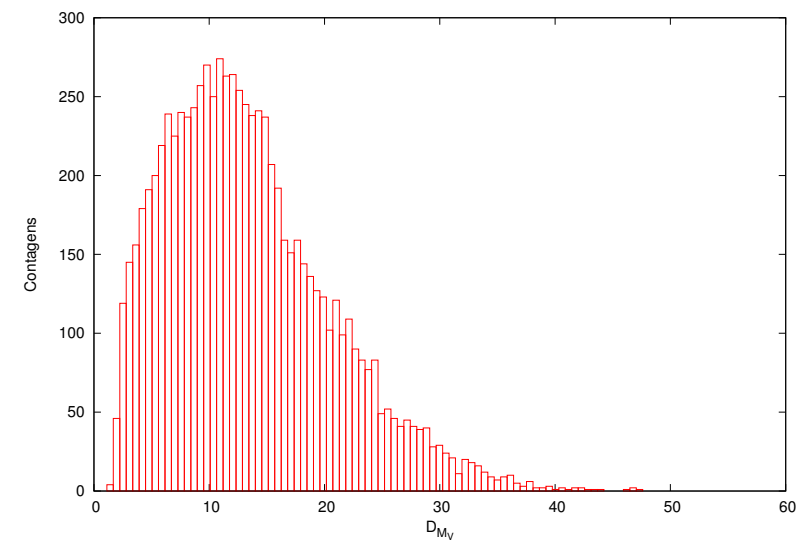

Figura 4.6: Distribuição de distâncias para as estrelas BS estimadas através das Equações 4.3 e 4.5. Nota-se a coerência entre esta distribuição e a distribuições encontrada por Deason et al. (2011), apresentada na Figura 4.5.

o disco galáctico e Z é a escala de altura. Partindo do centro galáctico, a coordenada $\mathrm{X}$ cresce no sentido do anticentro galáctico em direção ao Sol, enquanto Y aumenta no sentido de rotação da Galáxia. 

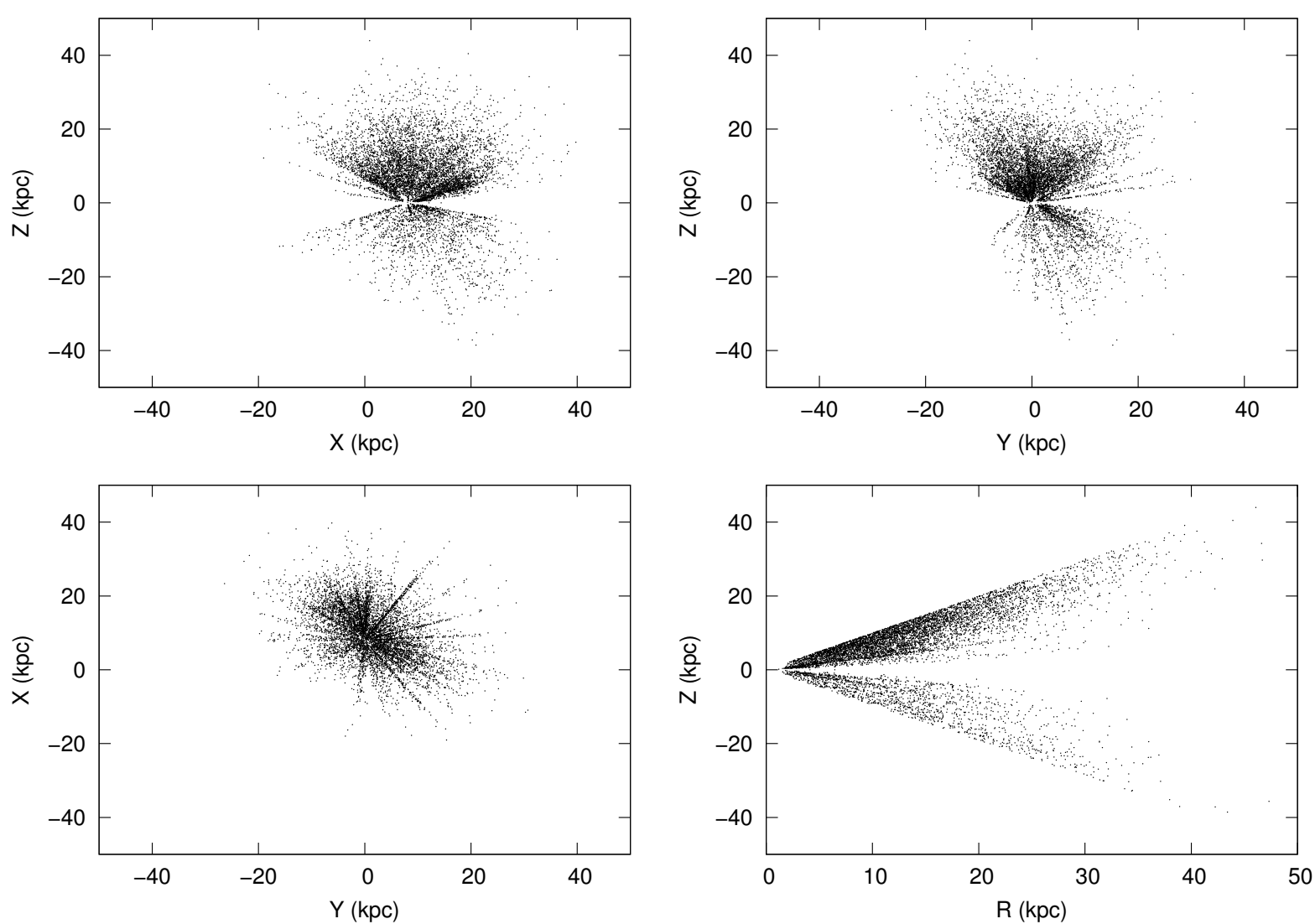

Figura 4.7: Distribuição das estrelas BS observadas na Galáxia em coordenadas galácticas XYZ. O plano XY contém o disco galáctico e Z define a distância até ele. Partindo do centro galáctico, a coordenada $\mathrm{X}$ aumenta no sentido do anticentro galáctico e $\mathrm{Y}$ aumenta no sentido de rotação da Galáxia.

\subsubsection{Tempo de vida na sequência principal}

No intervalo de temperaturas efetivas ao qual a amostra foi restrita, as estrelas BS selecionadas (tipo espectral A) possuem, teoricamente, massas entre $1.6 \mathrm{M}_{\odot}$ e $2.4 \mathrm{M}_{\odot}$ (Adelman, 2004). Com esses dados, pode-se estimar seu tempo de vida na sequência principal $\left(\tau_{S P}\right)$ em relação ao Sol, considerando-se que (Salaris e Cassisi, 2005):

$$
\tau_{S P}(\text { anos }) \approx 10^{10}\left(\frac{M}{M_{\odot}} \frac{L_{\odot}}{L}\right)
$$


e que

$$
\begin{aligned}
\frac{L}{L_{\odot}} & \approx 1.0\left(\frac{M}{M_{\odot}}\right)^{4.0} & \text { para } & 0.4 M_{\odot}<M<2.0 M_{\odot}, \mathrm{e} \\
\frac{L}{L_{\odot}} & \approx 1.5\left(\frac{M}{M_{\odot}}\right)^{3.5} & \text { para } & 2.0 M_{\odot}<M<20 M_{\odot},
\end{aligned}
$$

então

$$
\begin{aligned}
& \tau_{S P} \approx 10^{10}\left(\frac{M_{\odot}}{M}\right)^{-3.0} \quad \text { para } \quad 0.4 M_{\odot}<M<2.0 M_{\odot}, \mathrm{e} \\
& \tau_{S P} \approx 10^{10}\left(\frac{M_{\odot}}{M}\right)^{-2.5} \quad \text { para } \quad 2.0 M_{\odot}<M<20 M_{\odot},
\end{aligned}
$$

onde $L$ e $M$ representam a luminosidade e massa da estrela, respectivamente.

As estimativas de tempo de vida na sequência principal para as estrelas BS selecionadas são graficadas na Figura 4.8. De acordo com a idade estimada para as estrelas BS, duas possibilidades podem ser aventadas: ou devem existir correntes de estrelas de tipo A recém capturadas pela Galáxia (ao menos nos últimos 2.4Ganos), ou então, as estrelas BS devem ser oriundas da própria Galáxia. Pistas sobre essas possibilidades podem ser obtidas através das distribuições das velocidades desses objetos: caso existam estrelas BS extragalácticas, elas provavelmente possuem uma distribuição de velocidades diferente dos componentes galácticos.

\subsection{Distribuições de velocidades e a busca por correntes estelares}

As correntes estelares são primeiramente identificadas em diagramas HR construídos para regiões limitadas do céu, que mostram diferentes populações estelares (Belokurov et al., 2006, 2007). Entretanto, essa identificação e separação dentro de uma amostra homogênea de estrelas BS é difícil, como pode ser visto na Figura 4.9, pois elas se distribuem em torno de suas magnitudes aparentes típicas $(15<g<20)$. Além disso, elas são estão distribuídas homogeneamente pela Galáxia, com frequência naturalmente favorecida na região próxima ao Sol, em função do ponto de observação (painéis da Figura 4.7).

Assim, a análise das velocidades espaciais passa a ser a principal ferramenta de identificação de diferentes populações na amostra de estrelas BS. 


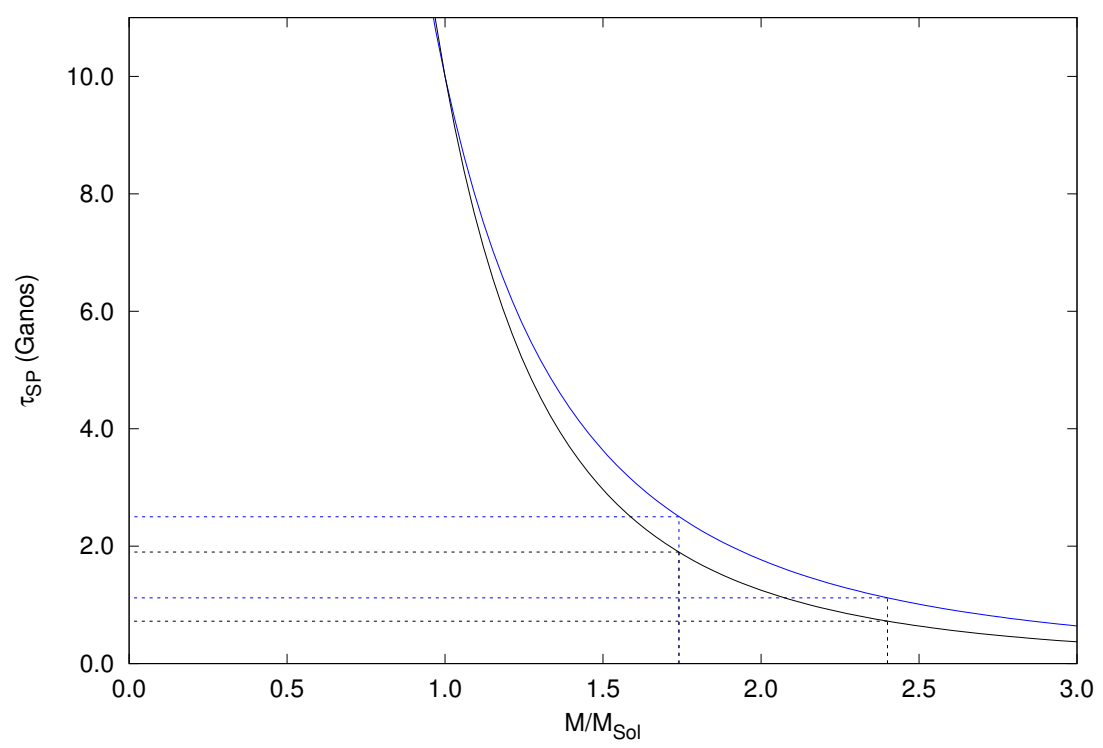

Figura 4.8: Relação teórica entre massa e tempo de vida na sequência principal em comparação ao Sol. As linhas verticais tracejadas mostram o intervalo de massa típico para estrelas A (com temperaturas efetivas equivalentes às estrelas BS selecionadas) e seu tempo de vida, identificados com linhas horizontais, para os casos onde $\mathrm{M}>2 \mathrm{M}_{\odot}$ (em azul) e $\mathrm{M}<$ $2 \mathrm{M}_{\odot}($ em preto). Elas se distribuem entre 1.1 e 2.4 bilhões de anos.

\subsubsection{Componentes de velocidades $U, V$ e $W$}

O sistema de velocidades $\mathrm{U}, \mathrm{V}$ e $\mathrm{W}$ corresponde aos movimentos no eixos $\mathrm{X}, \mathrm{Y}$ e Z, respectivamente, e com as mesmas orientações. Para definir um vetor de velocidade nesse sistema, são necessárias seis medidas para cada estrela (Mihalas e Binney, 1981): distância, duas coordenadas de localização no céu, dois movimentos próprios (denotados por $\mu$ ) e velocidade radial. Os movimentos próprios adotados são fornecidos pelo SDSS (Munn et al., 2004), extraídos do catálogo USNO-B (Monet et al., 2003). Neste caso especificamente, os valores do movimento próprio foram extraídos do DR9 (Ross et al., 2012). Eles possuem, quando diferentes de zero, erros muito grandes (Figura 4.10). Além disso, a completeza do catálogo é menor que $90 \%$ para $g<19.7$. Outros catálogos de movimentos próprios ou não possuem validade para $V>13$, ou não atendem a região do céu mapeada pelo SDSS. Mesmo assim, 6411 objetos da amostra possuem movimentos próprios medidos. Para converter as medidas angulares de movimento próprio em velocidades transversais, utiliza-se as seguintes relações: 


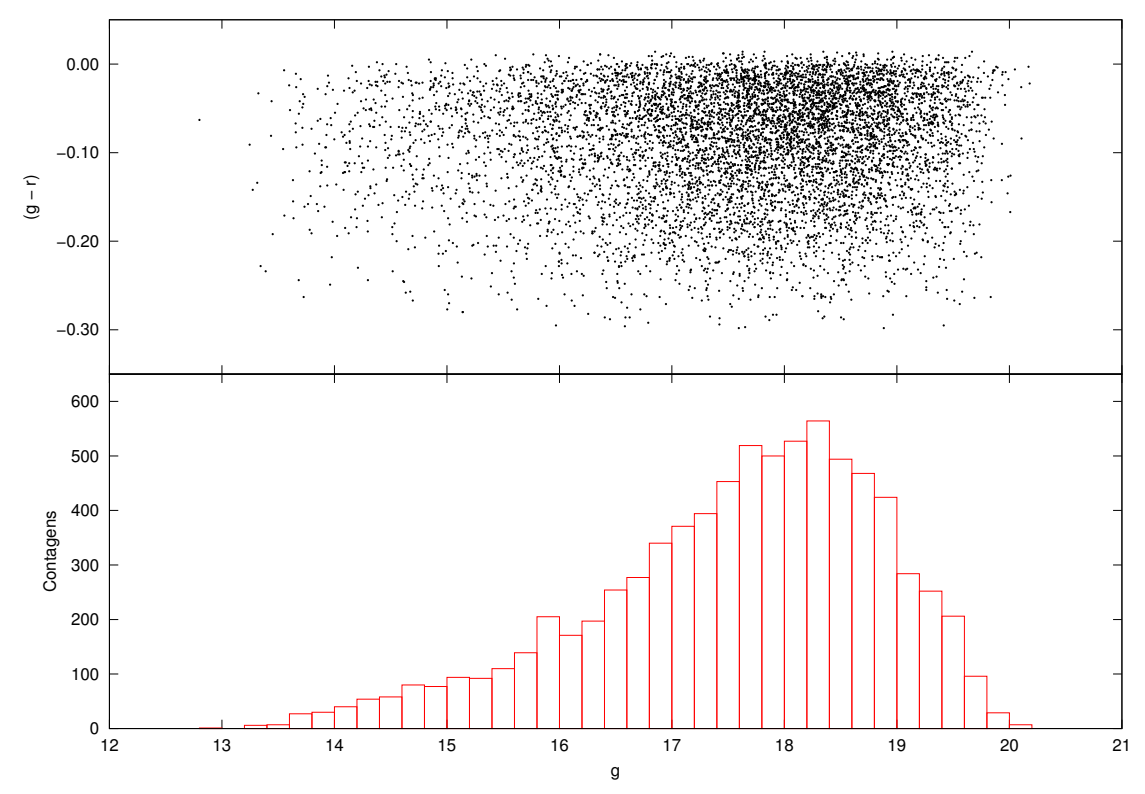

Figura 4.9: Na parte superior encontra-se o diagrama magnitude-cor das estrelas BS. É difícil distringuir, a partir de suas magnitudes, populações estelares diferentes ou superpostas, como mostra o painel inferior.

$$
\begin{gathered}
U=V_{R_{\odot}} \cos (l) \cos (b)+V_{l} \sin (l)+V_{b} \cos (l) \sin (b), \\
V=V_{R_{\odot}} \sin (l) \cos (b)+V_{l} \cos (l)+V_{b} \sin (l) \sin (b), \\
W=V_{R_{\odot}} \sin (b)+V_{b} \cos (b), \\
V_{l}=4.74 \cdot D \cdot \mu_{l}, V_{b}=4.74 \cdot D \cdot \mu_{b},
\end{gathered}
$$

onde $l$ e $b$ são, respectivamente, as coordenadas de longitude e latitude galácticas, $V_{l}$ e $V_{b}$ são as velocidades tangenciais a linha de visada associadas à elas, assim como os movimentos próprios $\mu_{l}$ e $\mu_{b}$. $V_{R_{\odot}}$ é a velocidade radial heliocêntrica e $D$ é a distância até o objeto.

A Figura 4.11 relaciona as velocidades U, V e W com a metalicidade buscando revelar grupos distintos através destes parâmetros. Entretanto, muitos objetos apresentam velocidades maiores que a velocidade de escape local da galáxia ( $500 \mathrm{~km} / \mathrm{s}$ Carney e Latham, 1987), ou seja, as velocidades U, V e W resultantes dos movimentos próprios fornecidos pelo SDSS parecem estar superestimadas. Sendo assim, elas não foram consideradas. Logo, a abordagem cinemática adotada na análise das estrelas BS passa a ser restrita apenas às velocidades radiais. Dentre os parâmetros calculados pelo SSPP, a velocidade radial é a 


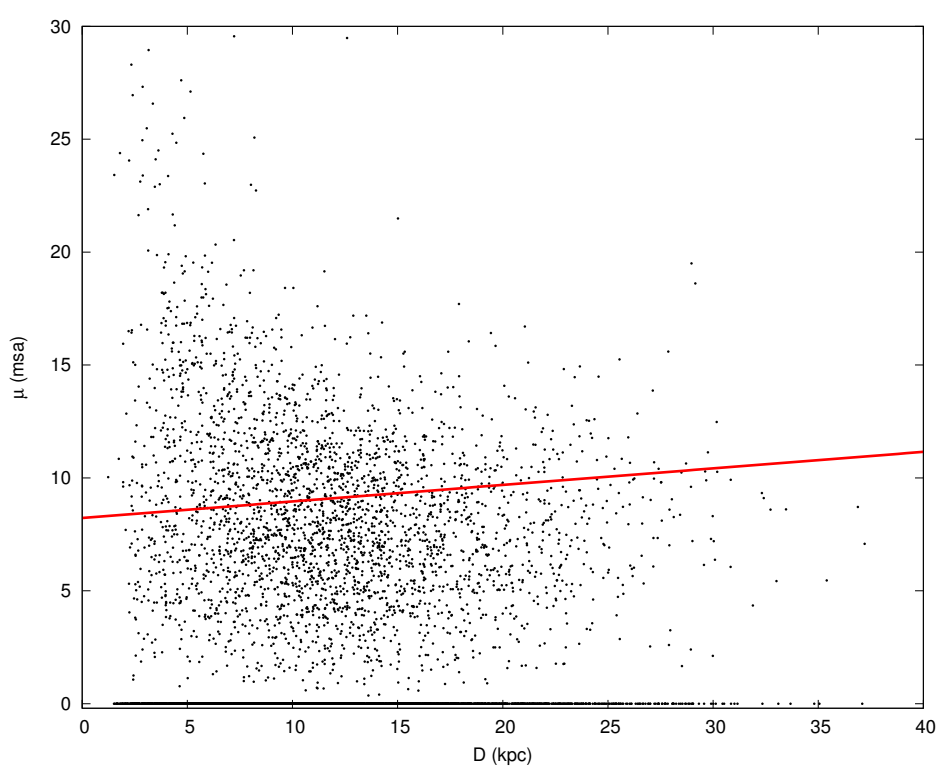

Figura 4.10: Movimento próprio total em função da distância D. Nota-se que os movimentos próprios são grandes demais para as distâncias estimadas, e também, na maior parte dos dados, os erros médios (linha vermelha) são maiores que o valores do movimento próprio.

que sofre menos influência da razão $\langle\mathrm{S} / \mathrm{R}\rangle$, tanto que, mesmo para estrelas com $\langle\mathrm{S} / \mathrm{R}\rangle$ $\sim 9.0$, os erros individuais na velocidade radial são sempre menores que $25 \mathrm{~km} / \mathrm{s}$ (Figura $4.15)$.

\subsubsection{Velocidades radiais heliocêntricas}

Através da velocidade radial heliocêntrica $\left(V_{R_{\odot}}\right)$ fornecida pelo SSPP e da distância ao plano galáctico Z, é possível analisar as estrelas BS distribuídas no disco e no halo galácticos. Essa análise exploratória procura por grupos de estrelas BS que se apresentem com diferentes padrões de velocidades, e que sejam identificadas claramente por distribuições de velocidades com mais de um pico. Para avaliar os diferentes ambientes galácticos, adota-se a separação entre o disco espesso e o halo interno como estando em $|\mathrm{Z}|$ $=5 \mathrm{kpc}$ (Figura 4.12), definido em trabalhos recentes que caracterizam os componentes da Galáxia (Carollo et al., 2008, 2010; Beers et al., 2012).

A Figura 4.13 mostra as distribuições de $V_{\mathrm{R}_{\odot}}$ à esquerda e $[\mathrm{Fe} / \mathrm{H}]$ à direita. De uma forma geral, não se nota a presença de multipicos (painéis superiores, todos os $|Z|$ ) e, aparentemente, nem para disco espesso (painéis centrais) e nem para o halo (painéis inferi- 

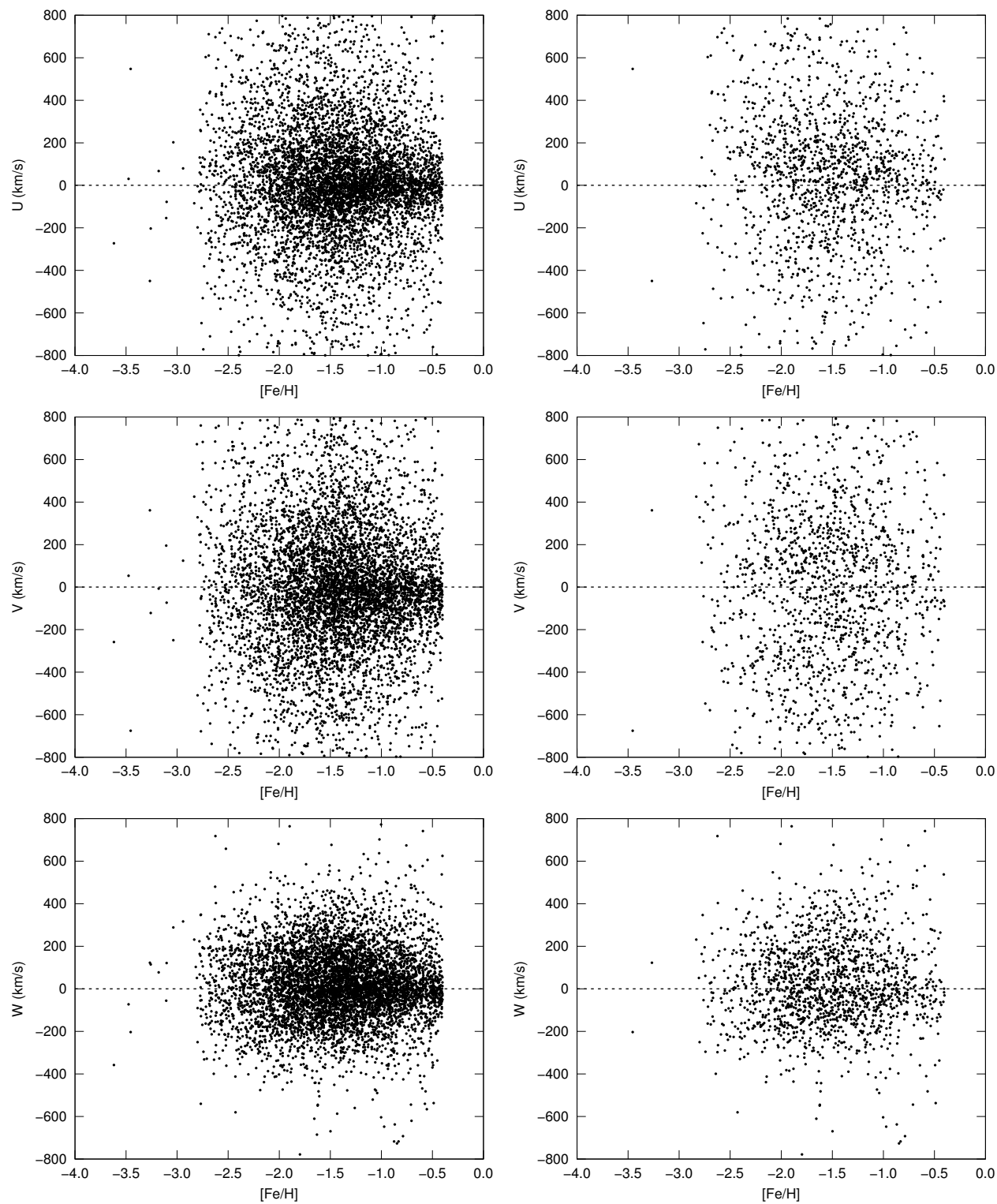

Figura 4.11: Velocidades U, V e W calculadas a partir dos movimentos próprios em função da metalicidade. Os painéis esquerdos mostram os valores obtidos para a toda a amostra e a direita vemos somente os objetos que possuem movimento próprio maior que seu erro. Em ambos os casos existem muitas estrelas que possuem velocidades superestimadas.

ores), quando vistos separadamente. Entretanto, em vista da alta dispersão de velocidades das estrelas presentes no halo, é interessante avaliá-lo em detalhes para verificar a existência de grupos superpostos, talvez com metalicidades diferentes.

Para tanto, prossegue-se com cortes transversais na direção Z da Galáxia, onde é possível examinar tanto a distribuição de metalicidades das estrelas BS (Figura 4.14), como a contribuição de cada componente estrutural da Galáxia: disco espesso - $[\mathrm{Fe} / \mathrm{H}]=$ 


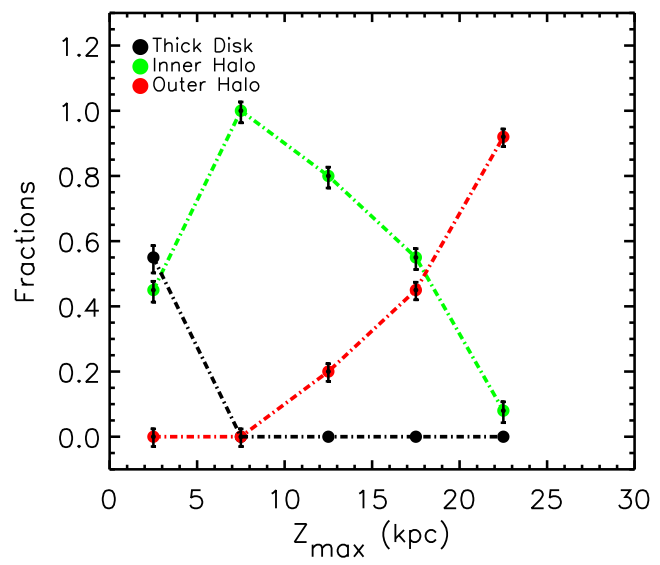

Figura 4.12: Fração estelar em função de $|\mathrm{Z}|$. O disco espesso é representado pela linha com pontos pretos, o halo interno em verde e o halo externo em vermelho. As frações não dependem da metalicidade. Figura retirada de Carollo et al. (2010).
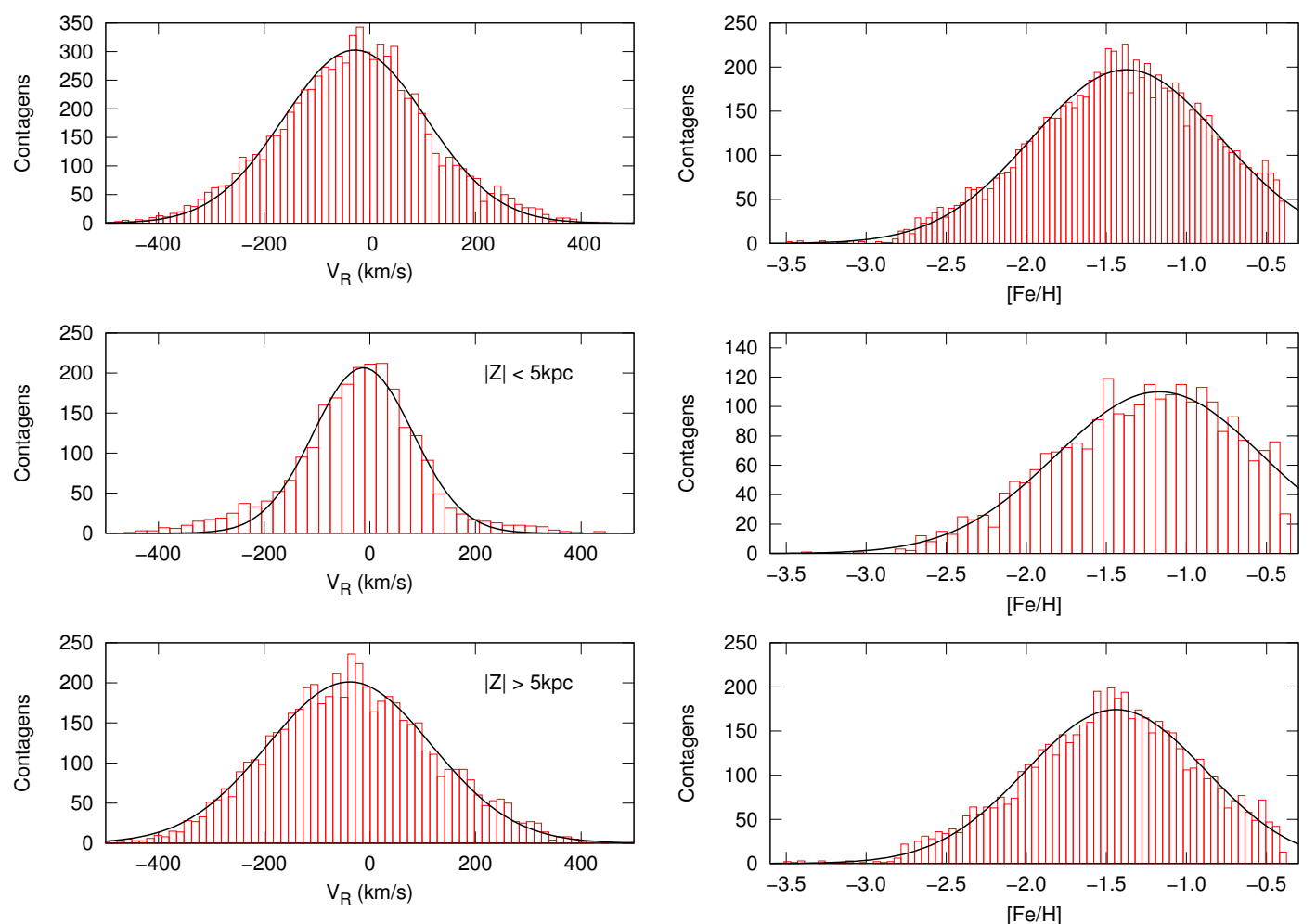

Figura 4.13: Distribuição das velocidades radiais heliocêntricas fornecidas pelo SSPP.

-0.6 ; disco espesso pobre em metal $-[\mathrm{Fe} / \mathrm{H}]=-1.3$; halo interno $-[\mathrm{Fe} / \mathrm{H}]=-1.6$; halo externo: $[\mathrm{Fe} / \mathrm{H}]=-2.2$, definidas por Carollo et al. (2008). A distribuição observada de metalicidade para as estrelas BS obedece o empobrecimento observado por Carollo et al. 
(2008, 2010) até atingir o halo externo. A partir dele, as estrelas aparentam enriquecer rapidamente (ver gráficos à direita na Figura 4.14) até atingir uma distribuição em torno de $[\mathrm{Fe} / \mathrm{H}] \sim-1.3$, mesma abundância encontrada para o disco espesso.

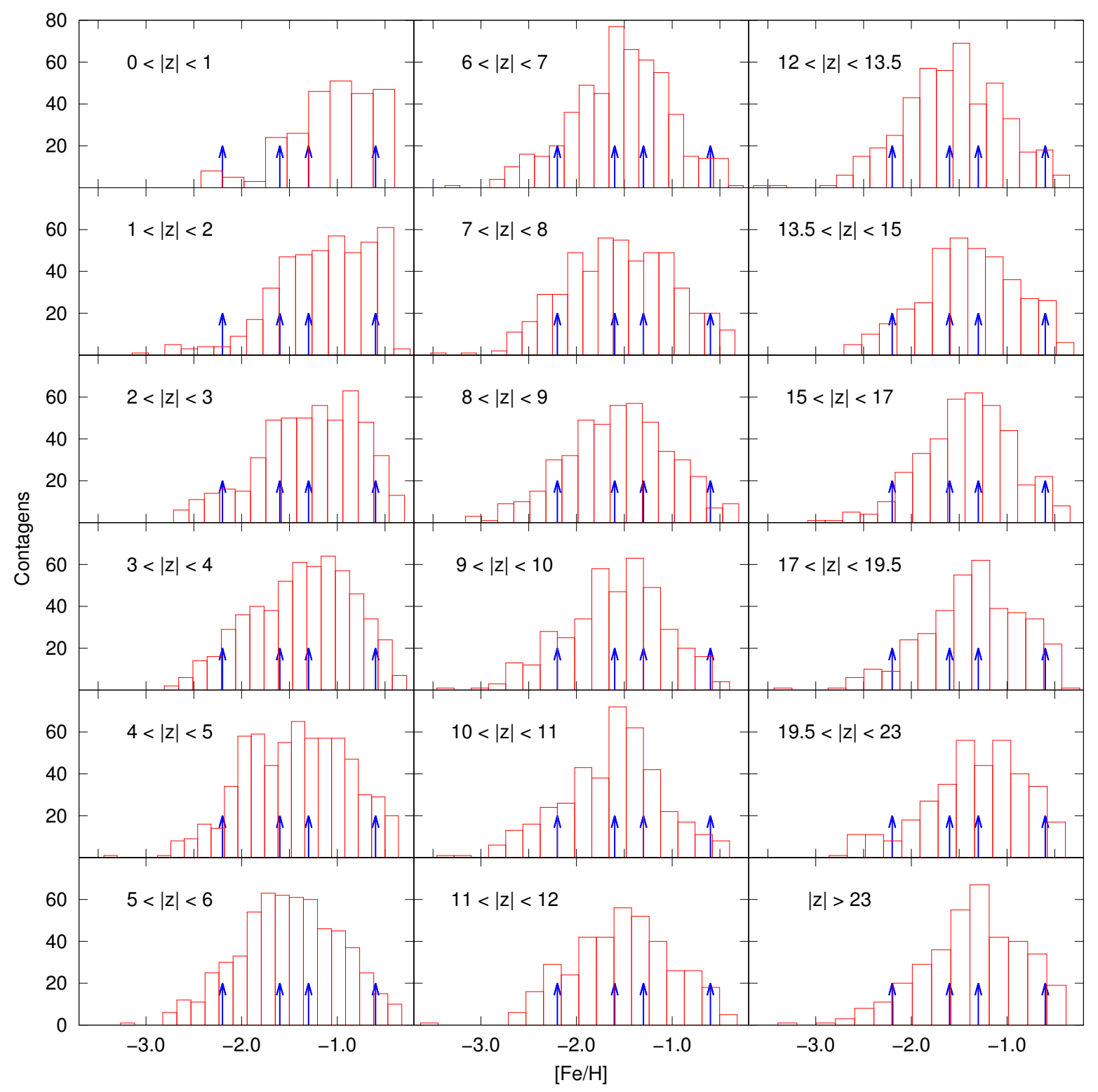

Figura 4.14: Distribuições de [Fe/H] em função da distância ao plano galáctico. As setas em azul identificam a metalicidade típica de cada componente galáctico: disco espesso $[\mathrm{Fe} / \mathrm{H}]=-0.6$; disco espesso pobre em metais $-[\mathrm{Fe} / \mathrm{H}]=-1.3$; halo interno $-[\mathrm{Fe} / \mathrm{H}]=$ -1.6; halo externo: $[\mathrm{Fe} / \mathrm{H}]=-2.2$. As distribuições de metalicidade concordam com Carollo et al. (2010) até o halo interno.

As estrelas encontradas no halo externo são as principais responsáveis por aumentar 
os erros nas distribuições da amostra. Suas incertezas maiores se devem às relações $\langle\mathrm{S} / \mathrm{R}\rangle$ baixas (Figura 4.15). No caso específico da metalicidade, as principais linhas metálicas que são utilizadas para as estimativas de abundância, dentre elas a linha K do Ca II, não são intensas para estrelas do tipo A, ocasionando erros maiores derivados de $[\mathrm{Fe} / \mathrm{H}]$ (Figura 4.15). Assim, qualquer consideração sobre a distribuição de metalicidades do halo externo utilizando as estrelas BS só pode ser feita com observações mais apuradas desses objetos. Além disso, deve-se ter em mente que a maioria das estrelas BS podem ter enriquecimento químico irregular devido à transferência de massa. Consequentemente, as avaliações das distribuições de velocidades radiais para os componentes do halo (interno ou externo) não levam em conta restrições na metalicidade.

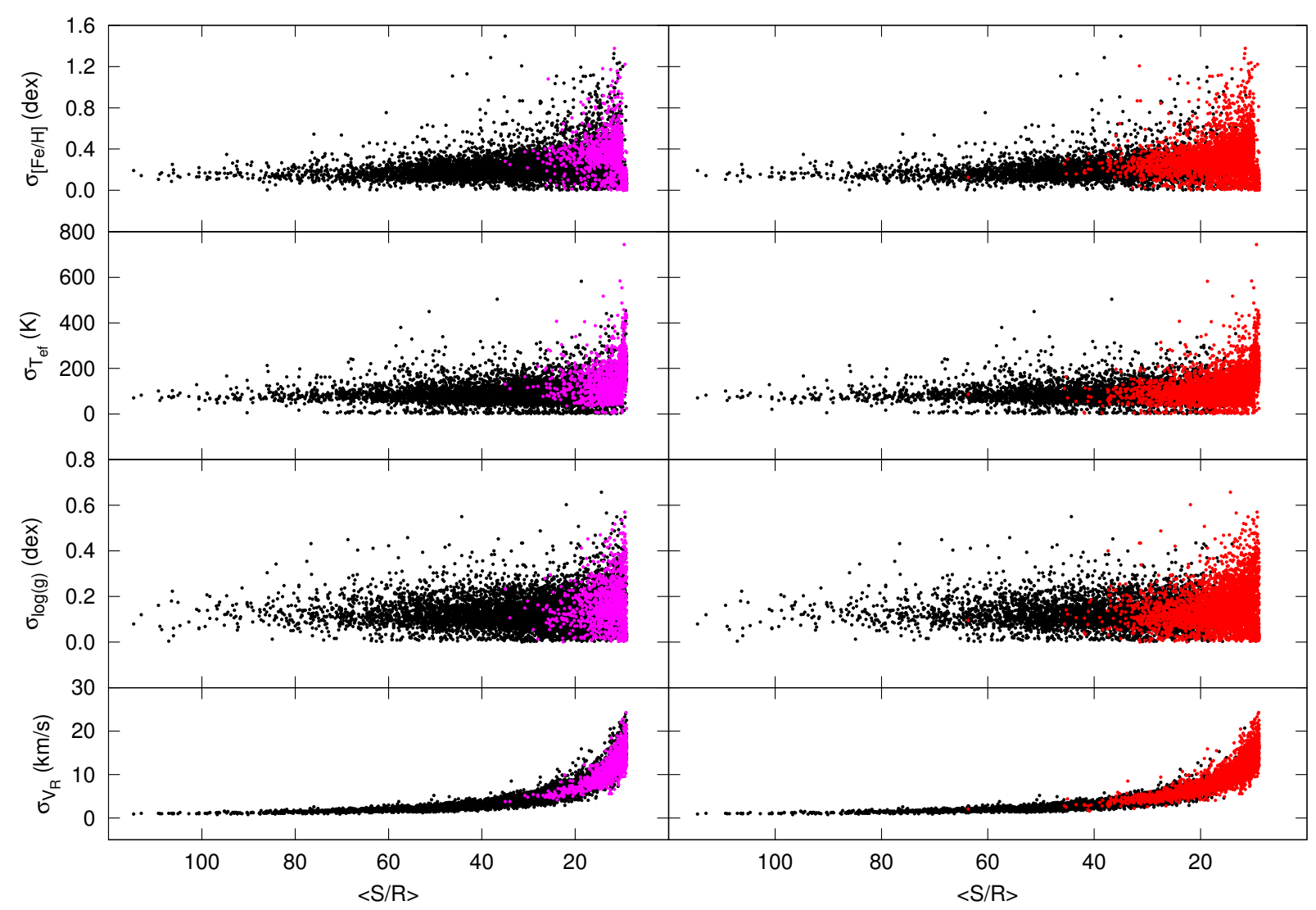

Figura 4.15: Erros dos parâmetros estelares ([Fe/H], $\mathrm{T}_{e f}, \log (\mathrm{g})$ e $\left.V_{\mathrm{R}_{\odot}}\right)$ em função da relação $\langle\mathrm{S} / \mathrm{R}\rangle$. O erro aumenta com a magnitude (pontos vermelhos: $g>18$ ) e consequentemente, para a maioria das estrelas do halo externo (pontos roxos: $|\mathrm{Z}|>17 \mathrm{kpc}$ ). A presença de pequenas populações com objetos de velocidades radiais destoantes não foi avaliada. Para tanto, é necessário reduzir a amostra de acordo com a precisão de interesse. 
Considerando o limite entre o halo interno e o halo externo em $|\mathrm{Z}|=17 \mathrm{kpc}$ (Figura 4.12), na Figura 4.16 apresenta-se a distribuição de velocidades radiais heliocêntricas para cada componente. Pode-se notar que o halo interno (painéis superiores) apresenta distribuição normal, enquanto o halo externo (painéis inferiores) possui duas populações superpostas.

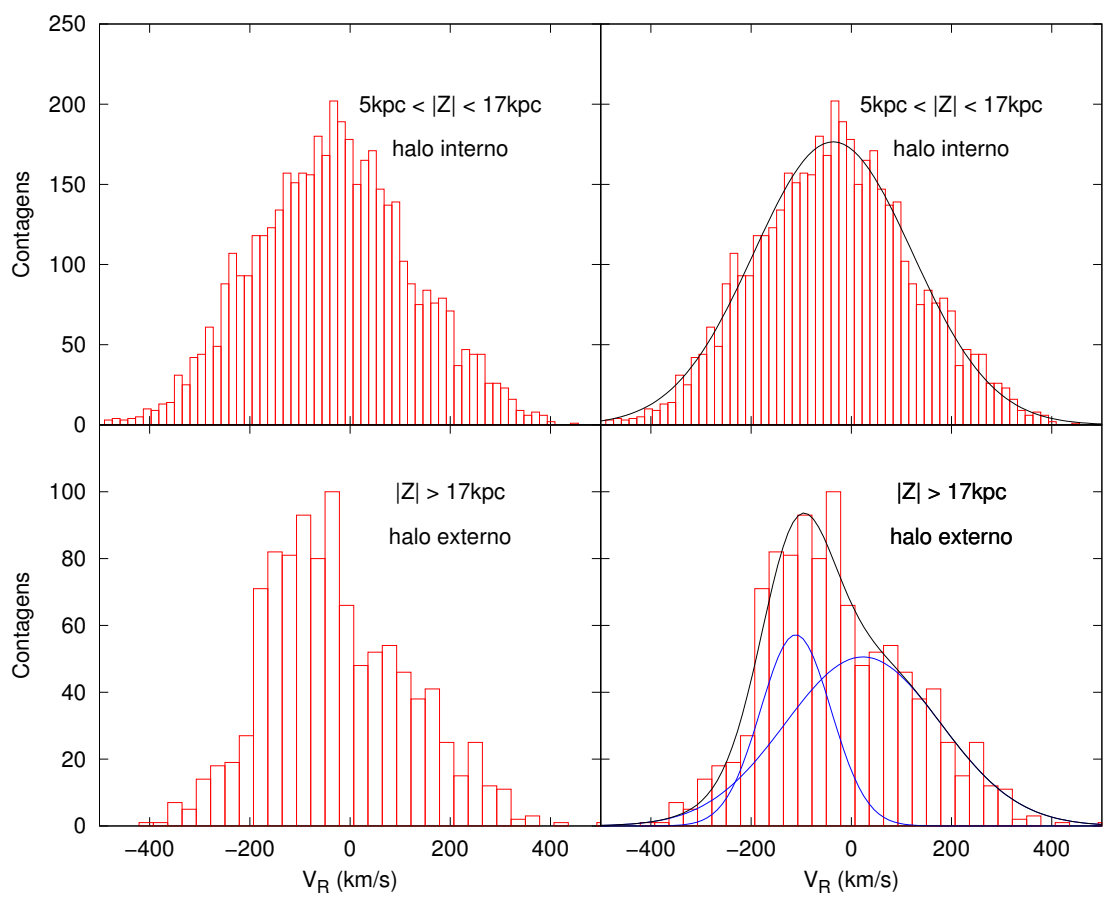

Figura 4.16: Distribuição das velocidades radiais heliocêntricas para fornecidas pelo SSPP. Os painéis superiores mostram as distribuições para o halo interno e painéis inferiores as distribuições do halo externo. Há superposição de grupos com diferentes velocidades radiais no halo externo.

As populações encontradas no halo externo são ajustadas por uma função gaussiana multipico que caracteriza duas componentes: um grupo com velocidade radial heliocêntrica negativa centralizado em $\left[V_{R_{\odot}}, \sigma_{V_{\odot}}\right] \approx[-111 \mathrm{~km} / \mathrm{s},+70 \mathrm{~km} / \mathrm{s}]$ e outro com velocidade radial posivita em $\left[V_{R_{\odot}}, \sigma_{V_{\odot}}\right]=[+17 \mathrm{~km} / \mathrm{s},+155 \mathrm{~km} / \mathrm{s}]$.

Porém, deve-se ter um cuidado adicional ao avaliar as velocidades radiais heliocêntricas, pois elas são referidas ao baricentro do Sistema Solar. As velocidades radiais obervadas na região $l>0^{\circ}$ são preferencialmente negativas e, para $l<0^{\circ}$, positivas. Além disso, o SDSS não faz um mapeamento uniforme do céu devido ao seu horizonte observável e, com isso, estrelas com coordenadas galácticas $l>0^{\circ}$ são mais frequentes que as estrelas para $l<$ 
$0^{\circ}$, fator responsável por deslocar as distribuições para valores de $V_{R_{\odot}}$ negativos, o mesmo efeito ocorre em relação à latitude galáctica, já que os objetos no hemisfério norte são melhor representados. Para verificar se as populações são resultado desse efeito de seleção, o halo externo é dividido com respeito aos hemisférios norte e sul galácticos (Figura 4.17). Com essa análise, nota-se que o pico negativo de distribuição de velocidades é explicado pela frequência predominante de estrelas na parte onde o SDSS tem maior visibilidade.
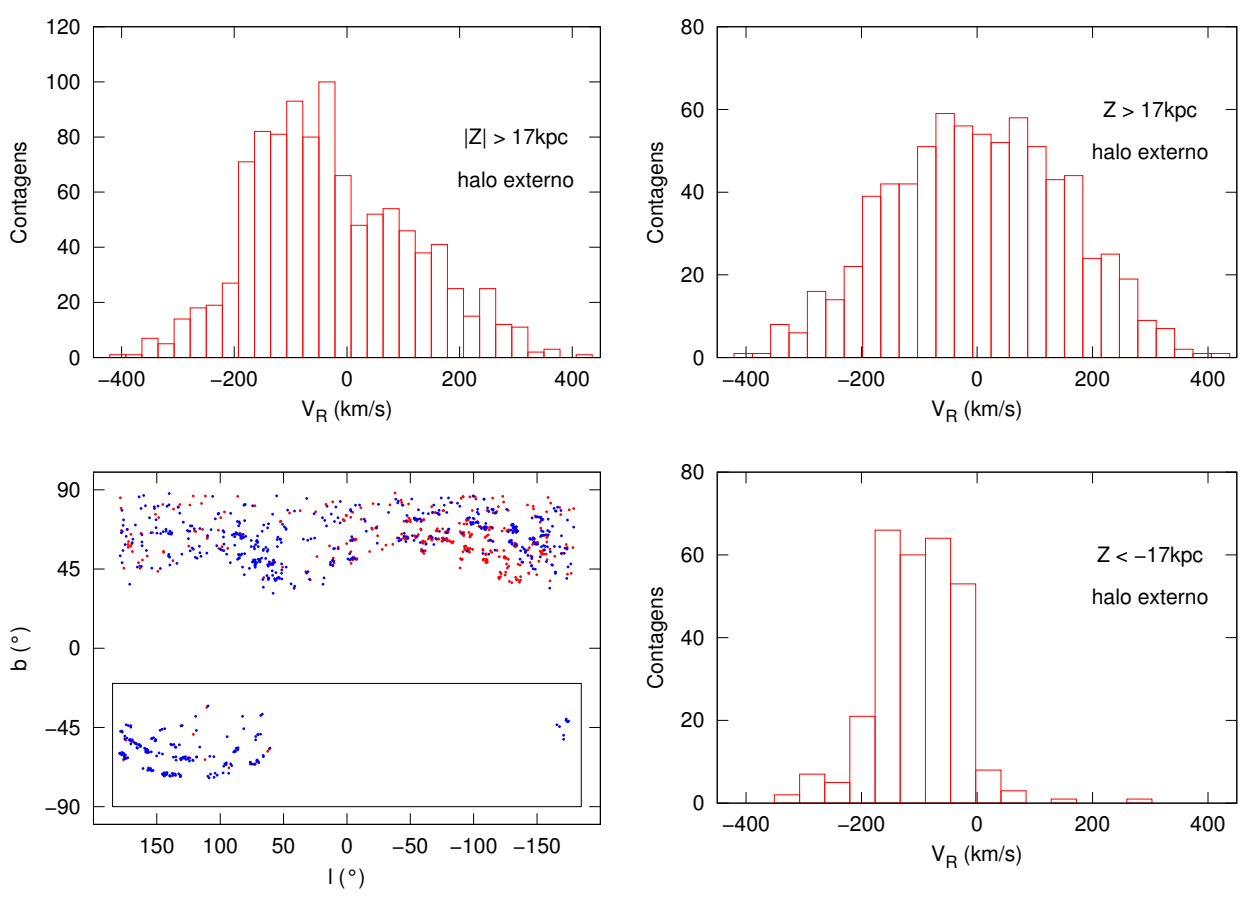

Figura 4.17: Distribuição das velocidades radiais heliocêntricas no halo externo. O painel superior esquerdo mostra a distribuição de $V_{R_{\odot}}$ para $|\mathrm{Z}|>17 \mathrm{kpc}$. Na parte inferior esquerda encontra-se a distribuição das estrelas BS em coordenadas galácticas. O retângulo destaca as estrelas com $\mathrm{Z}<-17 \mathrm{kpc}$, cuja distribuição de $V_{R_{\odot}}$ é vista no painel inferior direito. A distribuição de $V_{R_{\odot}}$ (painel superior direito) não apresenta duas populações superpostas para $\mathrm{Z}>17 \mathrm{kpc}$.

Assim, ao desconsiderar os efeitos da seleção nas regiões populosas do survey, as estrelas BS não apresentam uma população destoante da média de velocidades radiais relativas ao baricentro do Sistema Solar. Entretanto, devido aos efeitos de seleção aos quais o referencial heliocêntrico está submetido, deve-se adotar um ponto de vista diferente para analisar os movimentos. 


\subsubsection{Velocidades radiais galactocêntricas}

Para a análise subsequente, a velocidade radial heliocêntrica é convertida para o referêncial do Padrão Galactocêntrico de Repouso (PGR), denominada velocidade radial galactocêntrica $\left(V_{\mathrm{PGR}}\right)$. A transformada admite que o Sol está à 8kpc do centro galáctico e que se move com velocidade $\left(V_{\mathrm{PGR}}, V_{\phi}, V_{\mathrm{Z}}\right)=(-9 \mathrm{~km} / \mathrm{s}, 232 \mathrm{~km} / \mathrm{s}, 7 \mathrm{~km} / \mathrm{s})$ em relação a ele, e pode ser obtida via:

$$
V_{P G R}=V_{R_{\odot}}+232 \sin l \cos b+9 \cos l \cos b+7 \sin b
$$

A expressão anterior representa a combinação dos movimentos do Padrão Local de Repouso $\left(\mathrm{PLR}, V_{\phi}=220 \mathrm{~km} / \mathrm{s}\right.$ com $\left.V_{P G R}=V_{Z}=0\right)$ e dos movimentos do Sol (Mihalas e Binney, 1981). Neste referencial, também é interessante estimar a distância radial dos objetos até o centro galáctico. Isso pode ser obtido de acordo com a relação:

$$
R^{2}=\left(R_{\odot}-D \cos b \cos l\right)^{2}+(D \sin b)^{2}+(D \cos b \sin l)^{2}
$$

A distribuição das velocidades radiais galactocêntricas das estrelas BS, considerando a separação entre o halo e o disco espesso, pode ser visualizada na Figura 4.18. A distribuição do halo não é simples e é avaliada separadamente na Figura 4.19. De acordo com essa Figura, as velocidades radiais galactocêntricas das estrelas do halo apresentam um pico em torno de $V_{P G R} \sim-150 \mathrm{~km} / \mathrm{s}$, que se torna cada vez mais evidente com o aumento de R e |Z|. Pode-se notar a presença de uma população que possui velocidade radial coincidente com os valores encontrados recentemente por Koposov et al. (2012) para estrelas BHB, como mostra a Figura 4.20. Segundo esses autores, essa componente radial negativa se deve à corrente de Sagitário. Neste trabalho, ela está associada às estrelas BS com elevadas velocidades radiais galactocêntricas negativas, presentes nos dois hemisférios galácticos (Figura 4.21).

A Figura 4.21 mostra, em coordenadas celestes galácticas, a distribuição da amostra para $|\mathrm{Z}|>15 \mathrm{kpc}$ e a localização da corrente de Sagitário (retângulos pretos). Os objetos destacados em azul possuem velocidades radiais galactocêntricas entre $-180<V_{P G R}(\mathrm{~km} / \mathrm{s})$ $<-50$. Nota-se que, na análise utilizando as velocidades heliocêntricas, as estrelas presentes no hemisfério Sul galáctico foram excluídas devido à aparente observação preferencial 


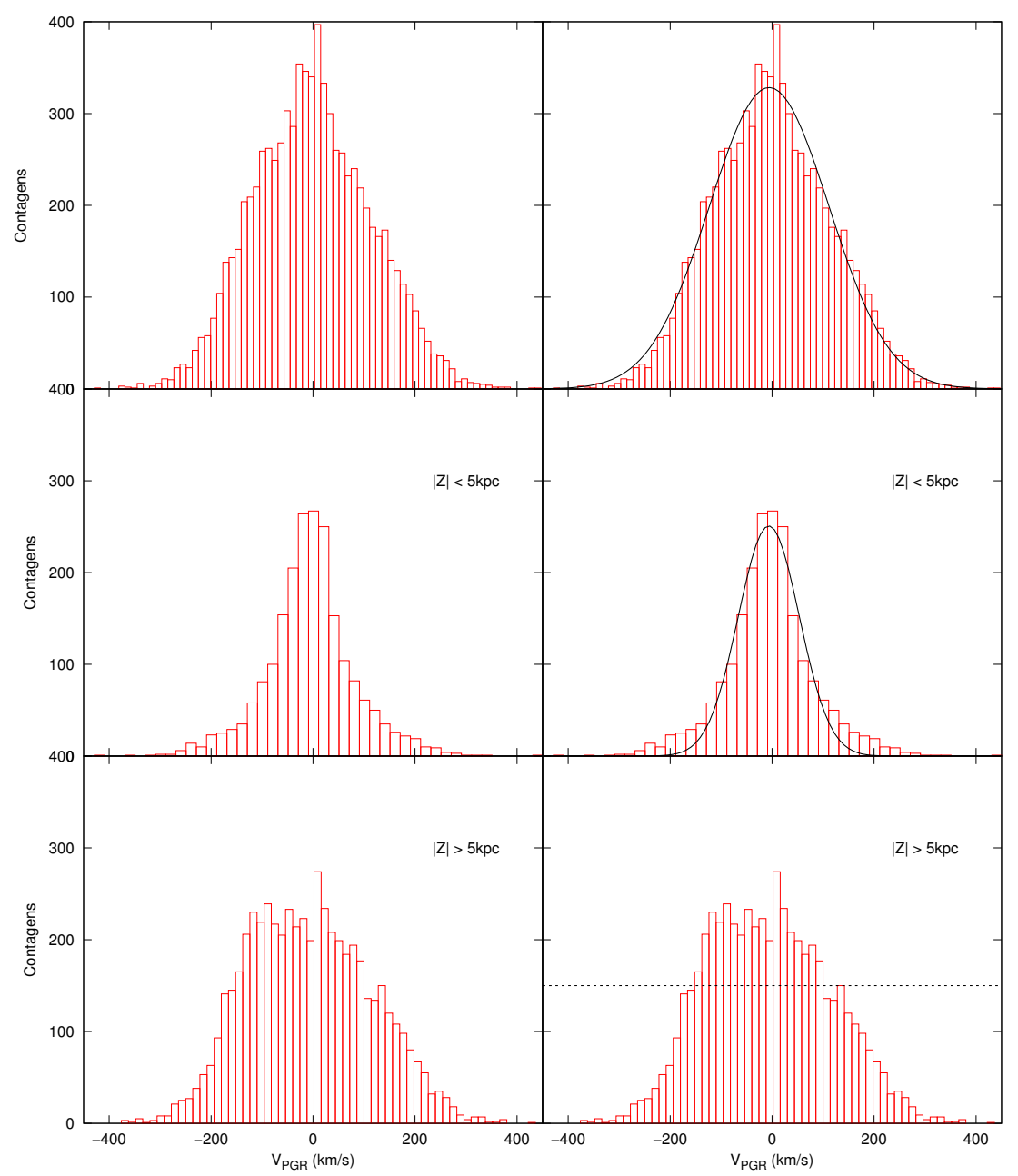

Figura 4.18: Distribuição das velocidades radiais galactocêntricas. Os painéis superiores mostram a distribuição de $V_{\mathrm{PGR}}$ para a amostra, os painéis centrais para $|\mathrm{Z}|<5 \mathrm{kpc}$ (disco espesso) e os inferiores, as distribuição para $|\mathrm{Z}|>5 \mathrm{kpc}$ (halo), a qual aparenta ser composta por ao menos duas populações. Os ajustes (à direita) mostram que as estrelas BS encontradas no disco espesso possuem distribuição simples em torno de zero, entretanto, a distribuição do halo não é simples, e portanto não pode ser representada por uma função gaussiana simples. A linha tracejada representa que nenhuma função foi ajustada nesse caso.

do SDSS. Na verdade, mesmo que o efeito ocorra, a identificação da corrente de Sagitário, poderia ter sido feita naquela ocasião.

Além da concordância entre as posições e velocidades radiais galactocêntricas, as estrelas destacadas com altas velocidades radiais negativas possuem distâncias coincidentes com a corrente de Sagitário (Figura 4.23). Elas se distribuem tem torno de $\mathrm{D}_{B S}=24.7 \pm 5.4$ kpc, e concordam com a distância até a corrente de Sagitário medida através de estrelas 

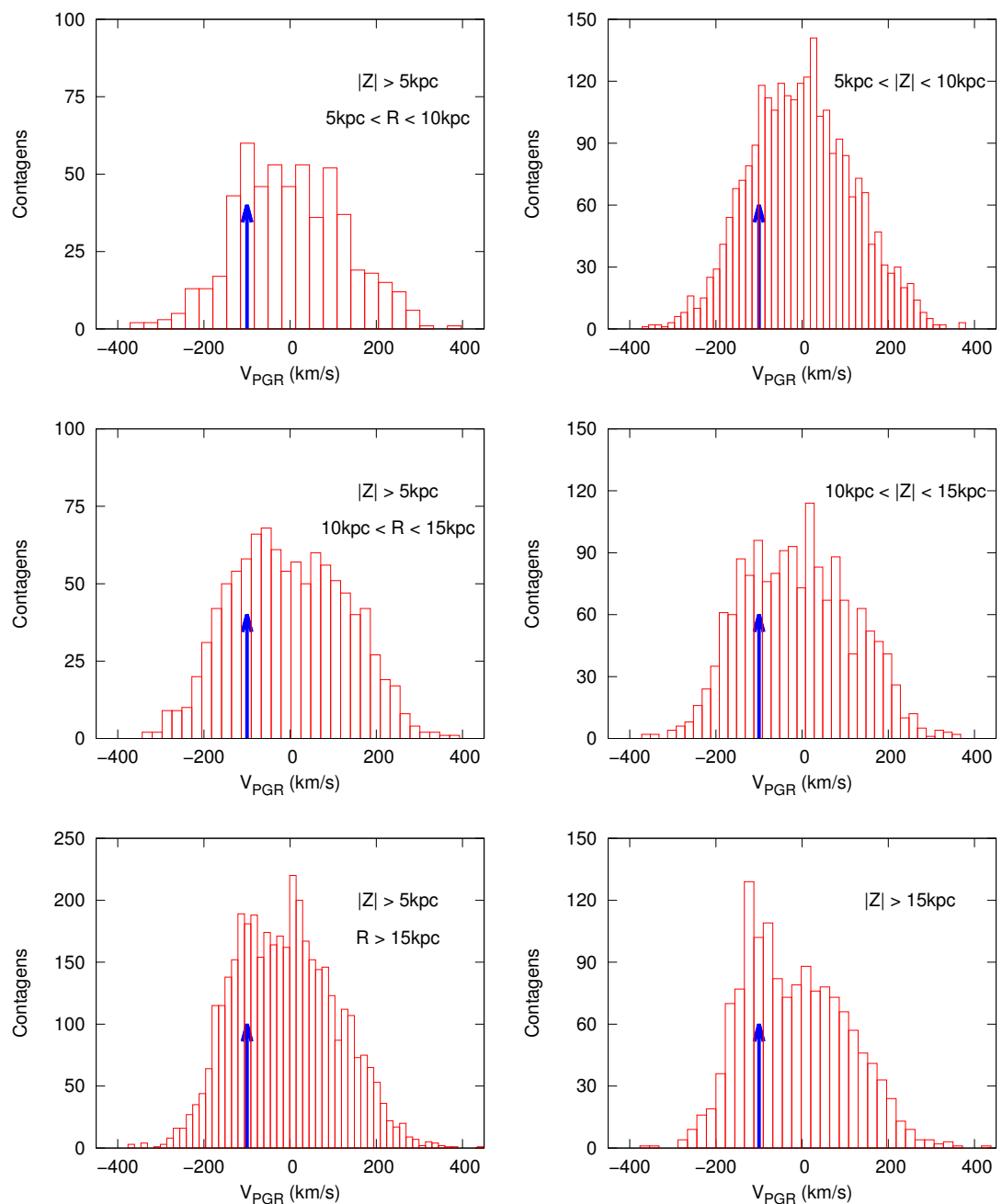

Figura 4.19: Os painéis à esquerda mostram como varia a distribuição de $V_{\mathrm{PGR}}$ para as estrelas do halo $(|Z|>5 \mathrm{kpc})$ em função da distância galactocêntrica $(\mathrm{R})$. Os painéis à direita mostram a variação da distribuição em função do módulo da distância ao plano galáctico $(|\mathrm{Z}|)$. Em ambos os casos, existe uma população de estrelas BS em torno de $V_{\mathrm{PGR}}=-150$ $\mathrm{km} / \mathrm{s}$ (identificada com uma seta azul) que se torna cada vez mais evidente para $\mathrm{R}$ e $|\mathrm{Z}|$ maiores.

RR Lyræ ( $\mathrm{D}_{S g}=26.1 \pm 5.6 \mathrm{kpc}$, Watkins et al., 2009).

Admitindo que as distribuições de velocidades radiais galactocêntricas são representadas por funções gaussianas e, que o excesso de estrelas encontrado em torno de $V_{\mathrm{PGR}}=$ $-150 \mathrm{~km} / \mathrm{s}$ se deve exclusivamente à corrente de Sagitário, a fração de estrelas BS encontrada com possível origem extragaláctica pode ser estimada entre 1000 a 1500 objetos, que representando $13-19 \%$ da amostra. 


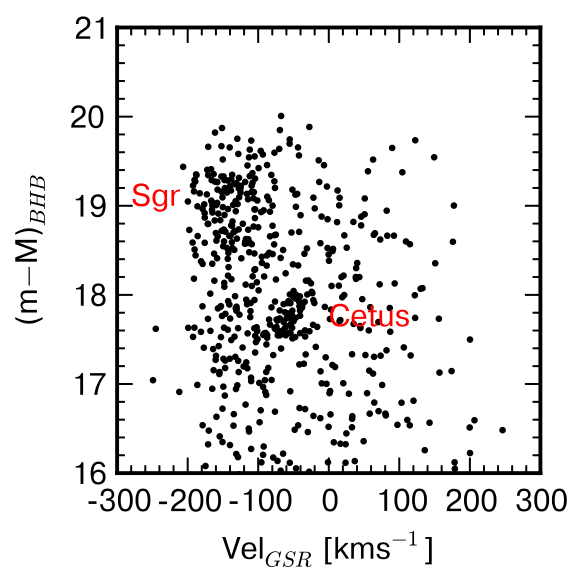

Figura 4.20: Velocidades galactocêntricas de estrelas BS e BHB da corrente de Sagitário no hemisfério Sul galáctico. Figura retirada de Koposov et al. (2012).

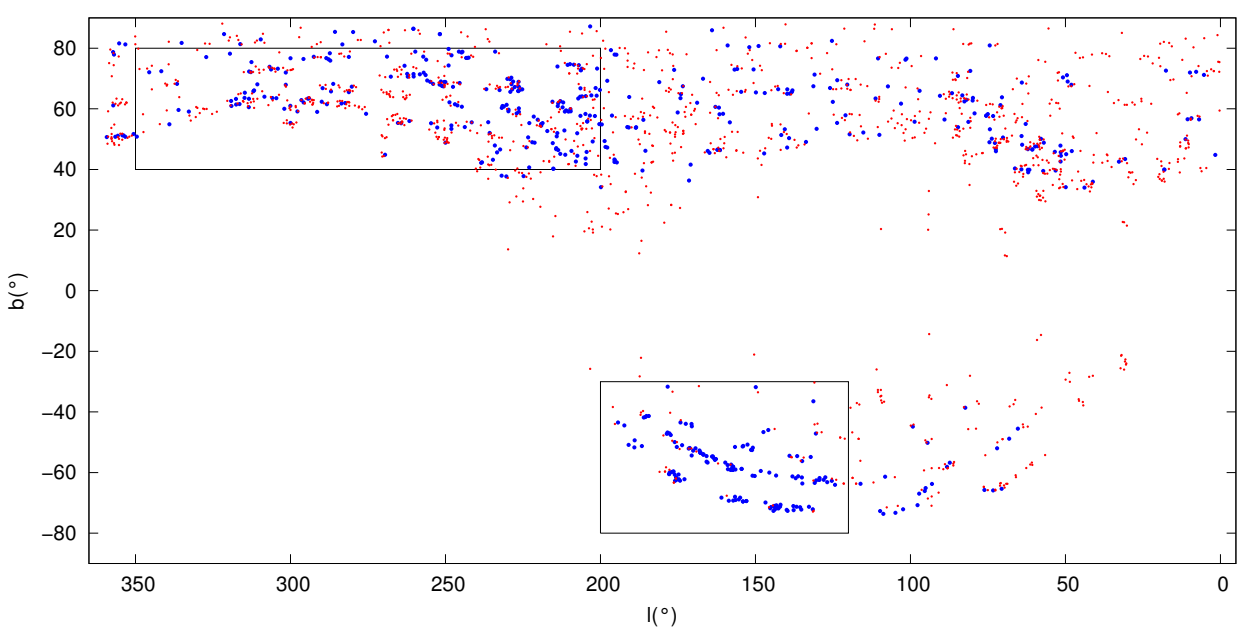

Figura 4.21: Destaque da corrente de Sagitário vista através de estrelas BS com $|\mathrm{Z}|>15$ kpc. As estrelas destacadas em azul possuem velocidades radiais galactocêntricas entre -180 $<V_{\mathrm{PGR}}(\mathrm{km} / \mathrm{s})<-50$. Muitas delas estão localizadas na região da corrente de Sagitário, evidenciada com retângulos pretos.

\subsection{Discussão}

Diante da presente análise, pôde-se verificar a predição de que $\sim 20 \%$ das estrelas BS observadas no campo podem ser originárias de outras galáxias resultado de interações passadas com a Galáxia (Preston et al., 1994; Preston e Sneden, 2000). Entretanto, a hipótese da origem extragaláctica foi proposta para explicar a presença de estrelas BS identificadas nas proximidades do Sol que não aparentavam pertencer ao disco. Quando se 


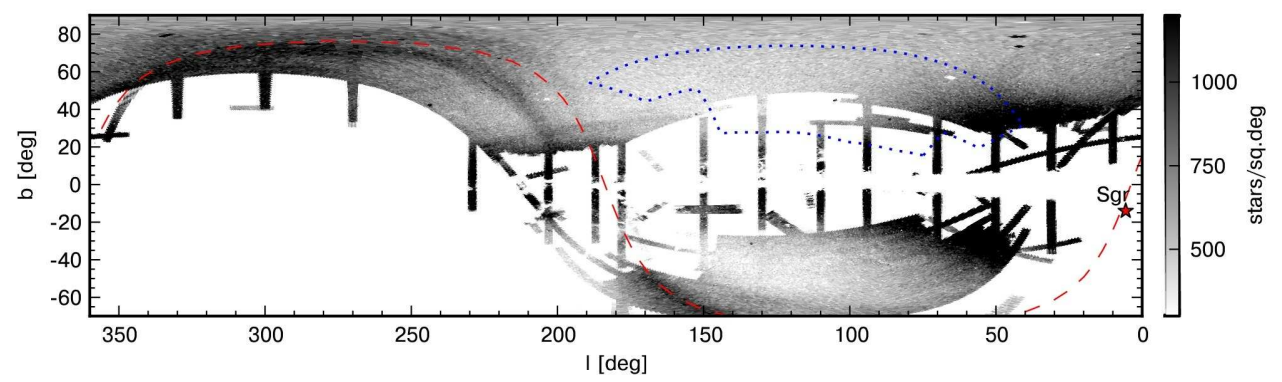

Figura 4.22: Corrente de Sagitário identificada por contraste de estrelas de sequência principal no ponto de turnoff com $0<(g-i)<0.7$ e $19.5<i<22.0$ (SDSS-DR8). A localização da galáxia anã remanescente da corrente de Sagitário é identicada pela estrela vermelha. A linha tracejada em vermelho marca a projeção do plano orbital da galáxia anã (consequentemente a posição da corrente). Figura retirada de Koposov et al. (2012).

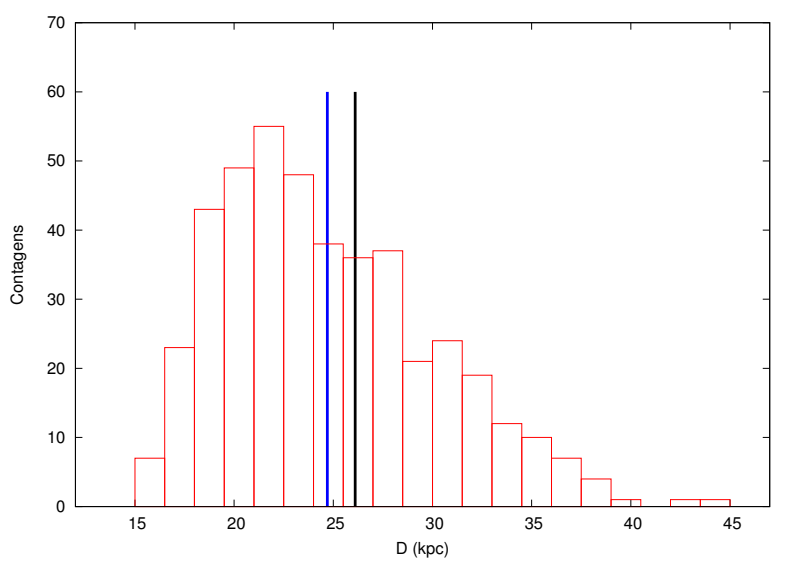

Figura 4.23: Distâncias das estrelas BS identificadas com altas velocidades radiais galactocêntricas negativas presentes nas regiões da corrente de Sagitário (vistas na Figura 4.21). A linha preta indica a distância até a corrente $\left(\mathrm{D}_{S g}=26.1 \pm 5.6 \mathrm{kpc}\right)$ calculada por Watkins et al. (2009) e a linha azul a distância estimada por este trabalho $\left(\mathrm{D}_{B S}=24.74 \pm 5.37 \mathrm{kpc}\right)$.

considera somente a a região dentro de $2 \mathrm{kpc}$ do Sol, não são observadas correntes massivas de estrelas BS no halo ou no disco. Curiosamente, ao mesmo tempo em que o resultado verifica a afirmação de Preston et al. (1994) que diz que 20\% das estrelas BS observadas no campo são extragalácticas, ele não condiz com a amostra adotada em seu trabalho, a qual avalia somente a vizinhança solar.

É importante ressaltar que o resultado encontrado não define se as estrelas BS identicadas na corrente de Sagitário são sistemas simples ou binários. Para o caso específico da corrente de Sagitário, que possui predominantemente estrelas com idades intermediárias 
(entre 6 e 9 bilhões de anos, segundo Bellazzini et al., 2006), as estrelas BS identicadas neste trabalho (idades menores que 3 bilhões de anos) são candidatas a estrelas BS formadas via transferência de massa por sistemas binários. Infelizmente, ainda não existem estudos que verifiquem a binariedade das estrelas BS extragalácticas, mesmo nas diferentes correntes da Galáxia.

A identificação de um número significativo de estrelas BS na corrente de Sagitário se deve, principalmente, à utilização dos critérios de seleção criados a partir dos parâmetros do SSPP. Graças à ele, estrelas com $g>18$ foram inseridas na amostra e avaliadas cinematicamente.

Portanto, dentro dos intervalos de distância nos quais as velocidades radiais foram avaliadas, conclui-se que as estrelas selecionadas no halo e no disco devem ter se originado dentro da Galáxia. A identificação de estrelas BS em todos os componentes da Galáxia, aponta para uma importante questão: quão raro deve ser esse fenômeno? A inclusão da transferência de massa (como fenômeno capaz de formar estrelas BS) modifica os modelos de síntese de populações estelares? Recentemente, alguns trabalhos dedicados à essas questões foram publicados (Chen e Han, 2009; Conroy e Gunn, 2010; Xin et al., 2011; Zhang et al., 2012) mostrando que a inclusão do fenômeno BS nesses modelos teóricos fornece estimativas mais precisas da idade de populações em aglomerados, e também, melhores ajustes da sequência principal e dos pontos de turnoff dos mesmos. 
Capítulo 5

\section{Conclusões e perspectivas}

De uma maneira concisa, é possível sumarizar os principais resultados encontrados neste trabalho, e também apresentar perspectivas para pesquisas futuras baseadas nas contribuições deixadas neste trabalho:

\subsection{Principais resultados}

- Busca por estrelas BS: Este trabalho demonstrou, através da combinação dos métodos clássicos de separação de estrelas anãs e gigantes com os parâmetros obtidos pelo SSPP, que é possível identificar estrelas BS com $g>18$. Como resultado, foram selecionados objetos a grandes distâncias do Sol, que até então eram descartados.

- Confiabilidade do SSPP para estrelas quentes: Foi verificada a validade, ao menos qualitativa, dos parâmetros estimados pelo SSPP, para estrelas com $\mathrm{T}_{\text {ef }}>7500 \mathrm{~K}$. Além disso, foi proposta uma automação no processo de identificação de estrelas BS internamente ao SSPP. O aumento da confiabilidade na separação entre estrelas de tipo A anãs e gigantes pode ajudar também na seleção de estrelas BHB, melhorando resultados já publicados que utilizam essas estrelas gigantes para calcular a forma da galáxia a longas distâncias (Deason et al., 2011) e até mesmo trabalhos que avaliam a matéria escura presente nela (Xue et al., 2008).

-Identificação de correntes através das velocidades radiais: A partir da capacidade do SSPP em distinguir estrelas BS de estrelas BHB para $g>18$, objetos mais distantes puderam ser avaliados. Dentre eles, vários objetos podem ser candidatos à estrelas BS na corrente de Sagitário. A quantidade de objetos pertencentes a corrente não foi avaliada em detalhes, mas a estimativa preliminar sugere que mais de 1000 objetos presentes na 
amostra sejam oriundos dela.

- Correntes dentro da Galáxia: Através da análise das velocidades radiais de estrelas BS, não foram observadas correntes estelares na parte interna da Galáxia, considerando-se os intervalos de distâncias avaliados.

\subsection{Perspectivas}

- Continuação da análise cinemática: A próxima geração de surveys certamente serão de grande ajuda para compreender os movimentos (GAIA) e propriedades físicas (LSST) das estrelas BS em um cenário maior e mais completo do que o atual.

- Identificação e quantificação de correntes de estrelas BS na Galáxia (em andamento): Os resultados obtidos nesse trabalho permitiram concluir que a identificação de correntes utilizando estrelas BS é possível. Através das distâncias e velocidades radiais, mais correntes podem ser estudadas. Entretanto, essa separação demanda tempo devido à quantidade de setores do céu que devem ser avaliados em detalhes.

-Dualidade do halo galáctico (em andamento): As estrelas BS galácticas podem ser identificadas mesmo à longas distâncias galácticas. Elas são mais numerosas que as estrelas BHB constituem uma nova classe de objetos que possibilita o estudo da dualidade do halo galáctico (Carollo et al., 2008, 2010).

- Milhares de estrelas BS na Galáxia: A amostra gerada por este trabalho reúne cerca de 7000 mil estrelas BS galácticas. Estudos estatísticos desses objetos, como por exemplo, sua frequência volumétrica (Preston e Sneden, 2000), podem revelar quão comum é o fenômeno BS na Galáxia e suas contribuições na observação de populações estelares em outras galáxias ou em modelos teóricos.

- Limites observacionais da frequência de estrelas BS em aglomerados globulares: As estrelas BS são menos frequentes em aglomerados globulares do que no campo galáctico (Preston e Sneden, 2000). A discrepância foi explicada através da destruição de sistemas binários (não muito cerrados) em ambientes estelares muito densos, devido ao excessivo número de interações gravitacionais. As estrelas BS de campo podem ser referência para o estudo da frequência dos sistemas binários em ambientes menos populosos, uma vez que é possível que exista relação entre a binaridade e a densidade dos aglomerados globulares. - Simulações de sistemas binários: Entre as 62 estrelas azuis pobres em metais obser- 
vadas por Preston e Sneden (2000), ao menos 45, se mostraram sistemas binários, sendo a maioria de longo período. Esses valores podem ser verificados teoricamente através de simulações estatísticas que comparem a capacidade de detecção de sistemas binários gerados aleatoriamente pela Galáxia com a fração observada por Preston e Sneden (2000). É possível que, dentro da resolução e dos métodos utilizados por Preston et al. (1994), a fração máxima identificável de sistemas binários tenha sido alcançada.

Como pode ser verificado ao longo do trabalho, o método de seleção de estrelas azuis tardias utilizando espectros em baixa resolução foi aprimorado, contribuindo com uma lista de $\sim 7000$ estrelas BS de campo e, além delas, $\sim 1000$ candidatas a estrelas BS identificadas na corrente de Sagitário. Esta contribuição, juntamente com os esforços teóricos e observacionais na área de evolução de sistemas binários, possuem papel importante na busca por descrições cada vez mais completas das origens das populações estelares e da estrutura da Galáxia. 


\section{Referências Bibliográficas}

Abt H. A., The spectra and ages of blue stragglers, ApJ, 1985, vol. 294, p. L103

Adelman S. J., The physical properties of normal A stars. In The A-Star Puzzle, vol. 224 of IAU Symposium, 2004, p. 1

Aihara H., Allende Prieto C., An D., Anderson S. F., Aubourg É., Balbinot E., Beers T. C., Berlind A. A., Bickerton S. J., Bizyaev D., Blanton M. R. et. al., The Eighth Data Release of the Sloan Digital Sky Survey: First Data from SDSS-III, ApJS, 2011, vol. 193, p. 29

Allende Prieto C., Sivarani T., Beers T. C., Lee Y. S., Koesterke L., Shetrone M., Sneden C., Lambert D. L., Wilhelm R. et. al., The SEGUE Stellar Parameter Pipeline. III. Comparison with High-Resolution Spectroscopy of SDSS/SEGUE Field Stars, AJ, 2008, vol. 136 , p. 2070

Arnold R., Gilmore G., Halo blue horizontal branch stars - Spectroscopy in two fields, MNRAS, 1992, vol. 257, p. 225

Beers T. C., Carollo D., Ivezić Ž., An D., Chiba M., Norris J. E., Freeman K. C., Lee Y. S. et. al., The Case for the Dual Halo of the Milky Way, ApJ, 2012, vol. 746, p. 34

Beers T. C., Christlieb N., The Discovery and Analysis of Very Metal-Poor Stars in the Galaxy, ARA\&A, 2005, vol. 43, p. 531

Beers T. C., Doinidis S. P., Griffin K. E., Preston G. W., Shectman S. A., Spectroscopy of hot stars in the Galactic halo, AJ, 1992, vol. 103, p. 267 
Beers T. C., Kage J. A., Preston G. W., Shectman S. A., Estimation of stellar metal abundance. I - Calibration of the CA II K index, AJ, 1990, vol. 100, p. 849

Beers T. C., Preston G. W., Shectman S. A., A search for stars of very low metal abundance. I, AJ, 1985, vol. 90, p. 2089

Beers T. C., Rossi S., Norris J. E., Ryan S. G., Shefler T., Estimation of Stellar Metal Abundance. II. A Recalibration of the Ca II K Technique, and the Autocorrelation Function Method, AJ, 1999, vol. 117, p. 981

Bellazzini M., Newberg H. J., Correnti M., Ferraro F. R., Monaco L., Detection of a population gradient in the Sagittarius stream, A\&A, 2006, vol. 457, p. L21

Belokurov V., Evans N. W., Irwin M. J., Lynden-Bell D., Yanny B., Vidrih S. et. al., An Orphan in the "Field of Streams", ApJ, 2007, vol. 658, p. 337

Belokurov V., Zucker D. B., Evans N. W., Gilmore G., Vidrih S., Bramich D. M. et. al., The Field of Streams: Sagittarius and Its Siblings, ApJ, 2006, vol. 642, p. L137

Benz W., Hills J. G., Three-dimensional hydrodynamical simulations of stellar collisions. I - Equal-mass main-sequence stars, ApJ, 1987, vol. 323, p. 614

Carney B. W., Latham D. W., The local galactic escape velocity. In IAU Symposium , vol. 117 of IAU Symposium, 1987, p. 39

Carney B. W., Latham D. W., Laird J. B., Metal-poor Field Blue Stragglers: More Evidence for Mass Transfer, AJ, 2005, vol. 129, p. 466

Carney B. W., Latham D. W., Laird J. B., Grant C. E., Morse J. A., A Survey of ProperMotion Stars. XIV. Spectroscopic Binaries among Metal-poor Field Blue Stragglers, AJ, 2001, vol. 122 , p. 3419

Carollo D., Beers T. C., Chiba M., Norris J. E., Freeman K. C., Lee Y. S., Ivezić Ž., Rockosi C. M., Yanny B., Structure and Kinematics of the Stellar Halos and Thick Disks of the Milky Way Based on Calibration Stars from Sloan Digital Sky Survey DR7, ApJ, 2010, vol. 712 , p. 692 
Carollo D., Beers T. C., Lee Y. S., Chiba M., Norris J. E., Wilhelm R., Sivarani T., Marsteller B., Munn J. A., Bailer-Jones C. A. L., Fiorentin P. R., York D. G., Two stellar components in the halo of the Milky Way, Nature, 2008, vol. 451, p. 216

Chen X., Han Z., Primordial binary evolution and blue stragglers, MNRAS, 2009, vol. 395, p. 1822

Clewley L., Warren S. J., Hewett P., Wilkinson M., Evans N. W., Mapping the Remote Milky Way Halo using BHB stars at 70 r 130 kpc. In Dark Matter in Galaxies, vol. 220 of IAU Symposium, 2004, p. 209

Clewley L., Warren S. J., Hewett P. C., Norris J. E., Peterson R. C., Evans N. W., Distant field blue horizontal branch stars and the mass of the Galaxy - I. Classification of halo A-type stars, MNRAS, 2002, vol. 337, p. 87

Conroy C., Gunn J. E., FSPS: Flexible Stellar Population Synthesis, Astrophysics Source Code Library, 2010, p. 10043

Deason A. J., Belokurov V., Evans N. W., The Milky Way stellar halo out to 40 kpc: squashed, broken but smooth, MNRAS, 2011, vol. 416, p. 2903

Ferraro F. R., Beccari G., Rood R. T., Bellazzini M., Sills A., Sabbi E., Discovery of Another Peculiar Radial Distribution of Blue Stragglers in Globular Clusters: The Case of 47 Tucanae, ApJ, 2004, vol. 603, p. 127

Ferraro F. R., Paltrinieri B., Fusi Pecci F., Cacciari C., Dorman B., Rood R. T., Buonanno R., Corsi C. E., Burgarella D., Laget M., HST observations of blue Straggler stars in the core of the globular cluster M 3., A\&A, 1997, vol. 324, p. 915

Ferraro F. R., Sabbi E., Gratton R., Piotto G., Lanzoni B., Carretta E., Rood R. T., Sills A., Fusi Pecci F., Moehler S., Beccari G., Lucatello S., Compagni N., Discovery of Carbon/Oxygen-depleted Blue Straggler Stars in 47 Tucanae: The Chemical Signature of a Mass Transfer Formation Process, ApJ, 2006, vol. 647, p. L53

Flynn C., Sommer-Larsen J., Christensen P. R., A method for identifying blue horizontal branch stars on the basis of spectra and photoelectric colours, MNRAS, 1994, vol. 267, p. 77 
Fukugita M., Ichikawa T., Gunn J. E., Doi M., Shimasaku K., Schneider D. P., The Sloan Digital Sky Survey Photometric System, AJ, 1996, vol. 111, p. 1748

Fusi Pecci F., Ferraro F. R., Corsi C. E., Cacciari C., Buonanno R., On the blue stragglers and horizontal branch morphology in Galactic globular clusters - Some speculations and a new working scenario, AJ, 1992, vol. 104, p. 1831

Gunn J. E., Carr M., Rockosi C., Sekiguchi M., Berry K., Elms B., de Haas E., Ivezić Ž., Knapp G., Lupton R., Pauls G. et. al., The Sloan Digital Sky Survey Photometric Camera, AJ, 1998, vol. 116, p. 3040

Gunn J. E., Siegmund W. A., Mannery E. J., Owen R. E., Hull C. L., Leger R. F., Carey L. N., Knapp G. R., York D. G., Boroski W. N., Kent S. M. et. al., The 2.5 m Telescope of the Sloan Digital Sky Survey, AJ, 2006, vol. 131, p. 2332

Habets G. M. H. J., Heintze J. R. W., Empirical bolometric corrections for the mainsequence, A\&AS, 1981, vol. 46, p. 193

Hills J. G., Day C. A., Stellar Collisions in Globular Clusters, Astrophys. Lett., 1976, vol. 17, p. 87

Iben Jr. I., On the binary star origin of blue stragglers, Mem. Soc. Astron. Italiana, 1986, vol. 57, p. 453

Ivezić Ž., Lupton R. H., Schlegel D., Boroski B., Adelman-McCarthy J., Yanny B., Kent S., Stoughton C., Finkbeiner D., Padmanabhan N. et. al., SDSS data management and photometric quality assessment, Astronomische Nachrichten, 2004, vol. 325, p. 583

Jofré P., Weiss A., The age of the Milky Way halo stars from the Sloan Digital Sky Survey, A\&A, 2011, vol. 533, p. A59

Kinman T. D., Suntzeff N. B., Kraft R. P., The structure of the galactic halo outside the solar circle as traced by the blue horizontal branch stars, AJ, 1994, vol. 108, p. 1722

Koposov S. E., Belokurov V., Evans N. W., Gilmore G., Gieles M., Irwin M. J., Lewis G. F., Niederste-Ostholt M., Peñarrubia J., Smith M. C., Bizyaev D., Malanushenko 
E., Malanushenko V., Schneider D. P., Wyse R. F. G., The Sagittarius Streams in the Southern Galactic Hemisphere, ApJ, 2012, vol. 750, p. 80

Lee Y. S., Beers T. C., Sivarani T., Allende Prieto C., Koesterke L., Wilhelm R., Re Fiorentin P. et. al., The SEGUE Stellar Parameter Pipeline. I. Description and Comparison of Individual Methods, AJ, 2008

Lee Y. S., Beers T. C., Sivarani T., Johnson J. A., An D., Wilhelm R., Allende Prieto C. et. al., The SEGUE Stellar Parameter Pipeline. II. Validation with Galactic Globular and Open Clusters, AJ, 2008

Leonard P. J. T., The Capture of Field Stars by Open Clusters: Can it Explain the Blue Stragglers?. In Bulletin of the American Astronomical Society, vol. 17 of Bulletin of the American Astronomical Society, 1985, p. 882

Leonard P. J. T., Stellar collisions in globular clusters and the blue straggler problem, AJ, 1989, vol. 98, p. 217

Liebert J., Young P. A., Arnett D., Holberg J. B., Williams K. A., The Age and Progenitor Mass of Sirius B, ApJ, 2005, vol. 630, p. L69

Lombardi C. J. J., Rasio F. A., Shapiro S. L., On blue straggler formation by direct collisions of main sequence stars, ApJ, 1995, vol. 445, p. L117

Lu P., Deng L.-C., Zhang X.-B., Modeling blue stragglers in young clusters, Research in Astronomy and Astrophysics, 2011, vol. 11, p. 1336

Lupton R., Gunn J. E., Ivezić Z., Knapp G. R., Kent S., Yasuda N., The SDSS Imaging Pipelines. In Astronomical Data Analysis Software and Systems X, vol. 238 of Astronomical Society of the Pacific Conference Series, 2001, p. 269

Maeder A., Evidences for a bifurcation in massive star evolution. The ON-blue stragglers, A\&A, 1987, vol. 178, p. 159

Mapelli M., Sigurdsson S., Colpi M., Ferraro F. R., Possenti A., Rood R. T., Sills A., Beccari G., The Contribution of Primordial Binaries to the Blue Straggler Population in 47 Tucanae, ApJ, 2004, vol. 605, p. L29 
Mapelli M., Sigurdsson S., Ferraro F. R., Colpi M., Possenti A., Lanzoni B., The radial distribution of blue straggler stars and the nature of their progenitors, MNRAS, 2006, vol. 373 , p. 361

Mathieu R. D., Latham D. W., The spatial distribution of spectroscopic binaries and blue stragglers in the open cluster M67, AJ, 1986, vol. 92, p. 1364

Mathys G., Properties of blue stragglers in young OB associations, A\&AS, 1987, vol. 71, p. 201

McCrea W. H., The interpretation of the Schwarzschild metric and the release of gravitational energy, Astrophysica Norvegica, 1964, vol. 9, p. 89

Mermilliod J.-C., Stellar content of young open clusters. I - Blue stragglers. II - Be stars, A\&A, 1982, vol. 109, p. 37

Mihalas D., Binney J., Galactic astronomy: Structure and kinematics /2nd edition/, 1981

Mihalas D. M., Stellar atmospheres., 1982

Momany Y., Held E. V., Saviane I., Zaggia S., Rizzi L., Gullieuszik M., The blue plume population in dwarf spheroidal galaxies. Genuine blue stragglers or young stellar population?, A\&A, 2007, vol. 468, p. 973

Monet D. G., Levine S. E., Canzian B., Ables H. D., Bird A. R., Dahn C. C. et. al., The USNO-B Catalog, AJ, 2003, vol. 125, p. 984

Munn J. A., Monet D. G., Levine S. E., Canzian B., Pier J. R. et. al., An Improved Proper-Motion Catalog Combining USNO-B and the Sloan Digital Sky Survey, AJ, 2004, vol. 127, p. 3034

Murray C. A., Vectorial astrometry, 1983

Nemec J. M., Cohen J. G., Blue straggler stars in the globular cluster NGC 5053, ApJ, 1989, vol. 336, p. 780

Nemec J. M., Harris H. C., Blue straggler stars in the globular cluster NGC 5466, ApJ, 1987, vol. 316 , p. 172 
Pier J. R., AB stars in the southern galactic halo. II - Spectroscopy and radial velocities, ApJS, 1983, vol. 53, p. 791

Pier J. R., Munn J. A., Hindsley R. B., Hennessy G. S., Kent S. M., Lupton R. H., Ivezić Ž., Astrometric Calibration of the Sloan Digital Sky Survey, AJ, 2003, vol. 125, p. 1559

Preston G. W., Beers T. C., Shectman S. A., The space density and kinematics of blue metal-poor main sequence stars near the solar circle, AJ, 1994, vol. 108, p. 538

Preston G. W., Sneden C., What Are These Blue Metal-Poor Stars?, AJ, 2000, vol. 120, p. 1014

Reid M. J., The distance to the center of the Galaxy, ARA\&A, 1993, vol. 31, p. 345

Roberts M. S., Dust and gas in globular clusters, AJ, 1960, vol. 65, p. 457

Ross A. J., Percival W. J., Carnero A., Zhao G.-b., Manera M., Raccanelli A. et. al., The Clustering of Galaxies in SDSS-III DR9 Baryon Oscillation Spectroscopic Survey: Constraints on Primordial Non-Gaussianity, ArXiv e-prints, 2012

Salaris M., Cassisi S., Evolution of Stars and Stellar Populations, 2005

Sandage A. R., The color-magnitude diagram for the globular cluster M 3., AJ, 1953, vol. 58 , p. 61

Sarajedini A., A Survey of Galactic Globular Clusters for Blue Straggler Stars. In Blue Stragglers, vol. 53 of Astronomical Society of the Pacific Conference Series, 1993, p. 14

Sarajedini A., Globular Cluster Photometry near the Turnoff: Blue Stragglers, Relative Ages, and the Horizontal Branch, PASP, 1994, vol. 106, p. 205

Sargent W. L. W., The Spectra and Masses of the Blue Horizontal-Branch Stars in M67, ApJ, 1968, vol. 152, p. 885

Sarna M. J., De Greve J.-P., Chemical Evolution of Algols, QJRAS, 1996, vol. 37, p. 11

Schild H., Berthet S., Blue stragglers and the OBN phenomenon, A\&A, 1986, vol. 162, p. 369 
Schlegel D. J., Finkbeiner D. P., Davis M., Maps of Dust Infrared Emission for Use in Estimation of Reddening and Cosmic Microwave Background Radiation Foregrounds, ApJ, 1998, vol. 500, p. 525

Sersic J. L., Atlas de galaxias australes, 1968

Sirko E., Goodman J., Knapp G. R., Brinkmann J., Ivezić Ž., Knerr E. J., Schlegel D., Schneider D. P., York D. G., Blue Horizontal-Branch Stars in the Sloan Digital Sky Survey. I. Sample Selection and Structure in the Galactic Halo, AJ, 2004, vol. 127, p. 899

Smecker-Hane T. A., Stetson P. B., Hesser J. E., Bursts of star formation in the Carina dwarf spheroidal galaxy. In American Astronomical Society Meeting Abstracts , vol. 26 of Bulletin of the American Astronomical Society, 1994, p. 1396

Sollima A., Lanzoni B., Beccari G., Ferraro F. R., Fusi Pecci F., The correlation between blue straggler and binary fractions in the core of Galactic globular clusters, A\&A, 2008, vol. 481 , p. 701

Sommer-Larsen J., Christensen P. R., Radial velocities of blue horizontal branch field stars in the outer Galactic halo, MNRAS, 1986, vol. 219, p. 537

Stoughton C., Lupton R. H., Bernardi M., Blanton M. R., Burles S., Castander F. J., Connolly A. J., Eisenstein D. J., Frieman J. A., Hennessy G. S. et. al.., Sloan Digital Sky Survey: Early Data Release, AJ, 2002, vol. 123, p. 485

Stryker L. L., Blue stragglers, PASP, 1993, vol. 105, p. 1081

Tucker D. L., Kent S., Richmond M. W., Annis J., Smith J. A., Allam S. S., Rodgers C. T., Stute J. L., Adelman-McCarthy J. K., Brinkmann J. et. al., The Sloan Digital Sky Survey monitor telescope pipeline, Astronomische Nachrichten, 2006, vol. 327, p. 821

Vilhu O., Detached to contact scenario for the origin of W UMa stars, A\&A, 1982, vol. 109, p. 17 
Watkins L. L., Evans N. W., Belokurov V., Smith M. C., Hewett P. C., Bramich D. M., Gilmore G. F., Irwin M. J., Vidrih S., Wyrzykowski Ł., Zucker D. B., Substructure revealed by RRLyraes in SDSS Stripe 82, MNRAS, 2009, vol. 398, p. 1757

Wheeler J. C., Blue stragglers as long-lived stars, ApJ, 1979, vol. 234, p. 569

Wilhelm R., Beers T. C., Gray R. O., Spectroscopy of Hot Stars in the Galactic Halo. II. The Identification and Classification of Horizontal-Branch and Other A-Type Stars, AJ, 1999

Wilhelm R., Beers T. C., Sommer-Larsen J., Pier J. R., Layden A. C., Flynn C., Rossi S., Christensen P. R., Spectroscopy of Hot Stars in the Galactic Halo. III. Analysis of a Large Sample of Field Horizontal-Branch and Other A-Type Stars, AJ, 1999

Williams I. P., The blue stars beyond the turn off point in some stellar clusters, Annales d'Astrophysique, 1964, vol. 27, p. 198

Willson L. A., Bowen G. H., Struck-Marcell C., Mass loss on the main sequence, Comments on Astrophysics, 1987, vol. 12, p. 17

Xin Y., Deng L., de Grijs R., Kroupa P., Simple stellar population models including blue stragglers, MNRAS, 2011, vol. 411, p. 761

Xue X. X., Rix H. W., Zhao G., Re Fiorentin P., Naab T., Steinmetz M., van den Bosch F. C., Beers T. C., Lee Y. S., Bell E. F. et. al., The Milky Way's Circular Velocity Curve to $60 \mathrm{kpc}$ and an Estimate of the Dark Matter Halo Mass from the Kinematics of 2400 SDSS Blue Horizontal-Branch Stars, ApJ, 2008, vol. 684, p. 1143

Yanny B., Newberg H. J., Kent S., Laurent-Muehleisen S. A., Pier J. R., Richards G. T., Stoughton C. et. al., Identification of A-colored Stars and Structure in the Halo of the Milky Way from Sloan Digital Sky Survey Commissioning Data, ApJ, 2000, vol. 540, p. 825

York D. G., Adelman J., Anderson Jr. J. E., Anderson S. F., Annis J., Bahcall N. A., Bakken J. A., Barkhouser R., Bastian S., Berman E. et. al., The Sloan Digital Sky Survey: Technical Summary, AJ, 2000, vol. 120, p. 1579 
Zhang Y., Han Z., Liu J., Zhang F., Kang X., Testing three derivative methods of stellar population synthesis models, MNRAS, 2012, vol. 421, p. 1678

Zhao C., Newberg H. J., Transformation from SDSS Photometric System to JohnsonMorgan-Cousins System in HK Survey, ArXiv Astrophysics e-prints, 2006

Zinn R., Searle L., The masses of the anomalous cepheids in the Draco system., ApJ, 1976, vol. 209, p. 734 
Apêndice 

Apêndice A

\section{Ajustes}

As Tabelas A.1, A.2, A.3 e A.4 mostram, respectivamente, os ajustes das Figuras 3.10, $3.21,3.23$ e 3.24 .

Tabela A.1 - Parâmetros do ajuste multipico com duas gaussianas (Equação B.3) - Figura 3.10.

\begin{tabular}{|c|c|c|c|}
\hline Parâmetro & Valor & Erro & Erro(\%) \\
\hline \hline $\mathrm{A} 1$ & 414.26 & 7.17 & $1.73 \%$ \\
$\mathrm{x}_{1}$ & 3.3851 & 0.0028 & $0.08 \%$ \\
$\sigma_{1}$ & 0.1380 & 0.0028 & $2.00 \%$ \\
\hline $\mathrm{A} 2$ & 340.97 & 6.14 & $1.801 \%$ \\
$\mathrm{x}_{2}$ & 4.3333 & 0.0039 & $0.09 \%$ \\
$\sigma_{2}$ & 0.1877 & 0.0039 & $2.083 \%$ \\
\hline
\end{tabular}

Tabela A.2 - Parâmetros do ajuste multipico com duas gaussianas (Equação B.3) - Figura 3.21.

\begin{tabular}{|c|c|c|c|}
\hline Parâmetro & Valor & Erro & Erro(\%) \\
\hline \hline $\mathrm{A} 1$ & 84.23 & 5.79 & $6.88 \%$ \\
$\mathrm{x}_{1}$ & 3.5727 & 0.0255 & $0.71 \%$ \\
$\sigma_{1}$ & 0.3280 & 0.0237 & $7.24 \%$ \\
\hline $\mathrm{A} 2$ & 294.00 & 8.53 & $2.90 \%$ \\
$\mathrm{x}_{2}$ & 4.2736 & 0.0049 & $0.12 \%$ \\
$\sigma_{2}$ & 0.2045 & 0.0043 & $2.08 \%$ \\
\hline
\end{tabular}


Tabela A.3 - Parâmetros do ajuste do polinômio de quarto grau visto na Figura $3.23-\mathrm{f}(\mathrm{x})=\mathrm{a} x^{4}+\mathrm{b} x^{3}$ $+\mathrm{c} x^{2}+\mathrm{d} x+\mathrm{e}$.

\begin{tabular}{|c|c|c|c|}
\hline Parâmetro & Valor & Erro & Erro(\%) \\
\hline \hline a & -9.55 & 4.32 & $45.23 \%$ \\
b & -27.58 & 13.69 & $49.63 \%$ \\
c & 87.42 & 15.82 & $18.09 \%$ \\
d & -49.05 & 7.86 & $16.02 \%$ \\
e & 8.64 & 1.41 & $16.28 \%$ \\
\hline
\end{tabular}

Tabela A.4 - Parâmetros do ajuste dos resíduos (Equação B.3) - Figura 3.24.

\begin{tabular}{|c|c|c|c|}
\hline Parâmetro & Valor & Erro & Erro(\%) \\
\hline \hline $\mathrm{A}$ & 278.42 & 5.60 & $2.01 \%$ \\
$\mathrm{x}_{0}$ & -0.028 & 0.0006 & $21.43 \%$ \\
$\sigma$ & 0.345 & 0.049 & $14.3 \%$ \\
\hline
\end{tabular}


Apêndice B

\section{Histogramas, gaussianas e o tratamento estatístico}

\section{B.1 Histograma e a escolha da raiz quadrada}

Um histograma é uma representação gráfica que mostra a impressão visual de uma distribuição de dados. É uma estimativa da distribuição de probabilidade de uma variável contínua. Ele consiste em tabular frequências, mostradas em retângulos adjacentes, erguidos sobre intervalos discretos, chamados bins. O bin tem altura igual a frequência de ocorrências no dado intervalo e sua largura depende, muitas vezes, do número total de dados.

Existem muitos modos de se determinar a largura de um bin, assim como o número total de bins para visualizar uma distribuição. Esse trabalho utiliza o método chamado escolha da raiz quadrada para estimar o número de bins em cada histograma construído. É o mesmo método (padrão) utilizado pelos programas de análise de dados. A escolha da raiz quadrada é interessante pois não assume antecipadamente que se conheça a tendência a ser representada, e aplica-se a qualquer tipo de distribuição, seja ela gaussiana ou não.

O método se resume simplesmente em extrair o número total de bins ideal $(k)$ através da raiz quadrada do número total de dados da distribuição $(N)$ :

$$
k=\sqrt{N}
$$

Para estimar a largura que cada bin deve possuir $(h)$, basta dividir o intervalo em que os dados se distribuem pelo número total de bins $k$ :

$$
h=\frac{\left|x_{\max }-x_{\min }\right|}{k} .
$$




\section{B.2 Gaussianas}

Muitas das distribuições apresentadas neste trabalho têm seu comportamento aproximado por uma função gaussiana (Figura B.1), descrita pela Equação B.3.

$$
G(x)=A e^{-\frac{\left(x-x_{0}\right)^{2}}{2 \sigma^{2}}},
$$

onde $A$ é a altura do pico, $x_{0}$ é a posição do centro (média) e $\sigma$ controla a largura da função (desvio padrão).

\section{B.2.1 Regra empírica}

A "regra dos $3 \sigma$ " (ou regra empírica) indica a fração do total da distribuição presente em um determinado intervalo de desvio padrão. Cerca de $68.27 \%$ dos valores encontram-se dentro de $1 \sigma$. Do mesmo modo, aproximadamente $95.45 \%$ dos valores encontram-se dentro de $2 \sigma$, e quase todos (99.73\%) encontram-se dentro de $3 \sigma$ (Figura B.1).

Matematicamente, esses fatos podem ser expressos da seguinte maneira. O parâmetro $x$ é uma observação de uma variável aleatória, com distribuição normal, $x_{0}$ é a média da distribuição (centro) e $\sigma$ é o seu desvio padrão (largura da distribuição). Assim, através desses parâmetros, pode-se calcular a probabilidade $(\mathrm{P})$ de $x$ ser encontrado em torno de seu valor médio, vista na Tabela B.1. Os intervalos de probabilidade são chamados de intervalos de confiança.

Tabela B.1 - Probabilidade (P) de $x$ ser encontrado em torno de seu valor médio (em \%).

$$
\begin{array}{|c|}
\hline \mathrm{P}\left(x_{0}-\sigma \leq \mathrm{x} \leq \sigma+x_{0}\right) \simeq 0.6827=68.27 \% \\
\hline \mathrm{P}\left(x_{0}-2 \sigma \leq \mathrm{x} \leq 2 \sigma+x_{0}\right) \simeq 0.9545=95.45 \% \\
\hline \mathrm{P}\left(x_{0}-3 \sigma \leq \mathrm{x} \leq 3 \sigma+x_{0}\right) \simeq 0.9973=99.73 \% \\
\hline
\end{array}
$$

Assim, a confiança de que $x$ encontra-se dentro de $3 \sigma$ é $99.73 \%$, e ao mesmo tempo, a confiança é de $0.27 \%$ que esteja fora de $3 \sigma$. Portanto, valores medidos fora dessa margem $(3 \sigma)$ possuem pouca confiança em pertencer à distribuição. 


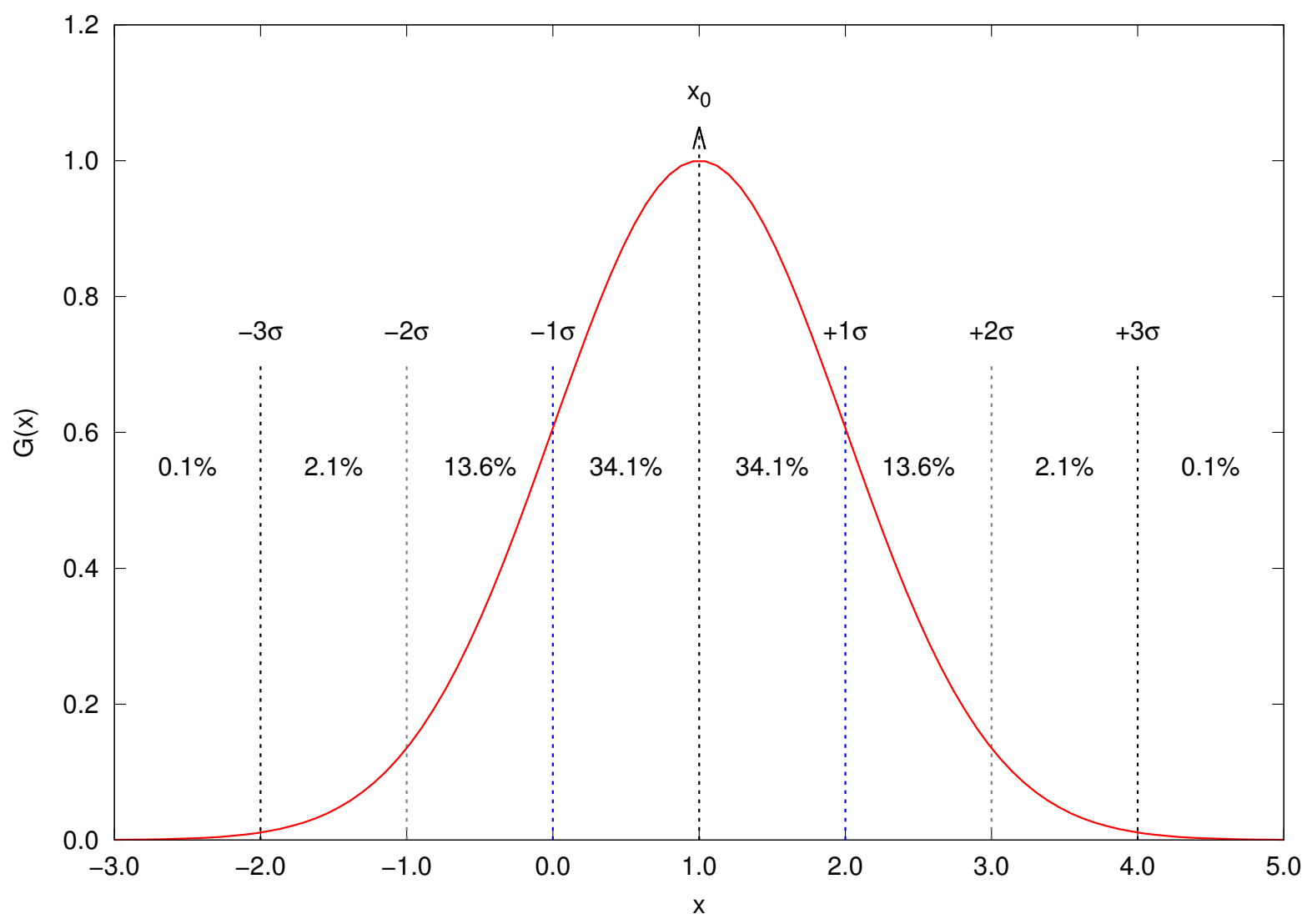

Figura B.1: Função gaussiana com $\mathrm{A}=\mathrm{x}_{0}=\sigma=1$. A cada desvio padrão, são vistas as probabilidades de se observar a variável aleatória. 